\title{
THE XMM CLUSTER SURVEY: THE STELLAR MASS ASSEMBLY OF FOSSIL GALAXIES
}

\author{
Craig D. Harrison $^{1}$, Christopher J. Miller ${ }^{1}$, Joseph W. Richards ${ }^{2}$, E. J. Lloyd-Davies ${ }^{3}$, Ben Hoyle ${ }^{4,5,6}$, \\ A. Kathy Romer ${ }^{3}$, Nicola Mehrtens ${ }^{3}$, Matt Hilton ${ }^{7,8}$, John P. Stott $^{9,10}$, Diego Capozzi ${ }^{9}$, Chris A. Collins ${ }^{9}$, \\ Paul-James Deadman ${ }^{2}$, Andrew R. Liddle ${ }^{3}$, Martin Sahlén ${ }^{11}$, S. Adam Stanford ${ }^{12,13}$, and Pedro T. P. Viana ${ }^{14,15}$ \\ ${ }^{1}$ Department of Astronomy, University of Michigan, Ann Arbor, MI 48109, USA; craigha@umich.edu \\ ${ }^{2}$ Center for Time Domain Informatics, University of California, Berkeley, CA 94720, USA \\ ${ }^{3}$ Astronomy Centre, University of Sussex, Falmer, Brighton BN1 9QH, UK \\ ${ }^{4}$ Institute of Sciences of the Cosmos (ICCUB) and IEEC, Physics Department, University of Barcelona, Barcelona 08024, Spain \\ ${ }^{5}$ CSIC, Consejo Superior de Investigaciones Cientificas, Serrano 117, Madrid 28006, Spain \\ ${ }^{6}$ Helsinki Institute of Physics, P.O. Box 64, FIN-00014 University of Helsinki, Helsinki, Finland \\ ${ }^{7}$ Astrophysics \& Cosmology Research Unit, School of Mathematical Sciences, University of KwaZulu-Natal, Private Bag X54001, Durban 4000, South Africa \\ ${ }^{8}$ School of Physics \& Astronomy, University of Nottingham, Nottingham NG7 2RD, UK \\ ${ }^{9}$ Astrophysics Research Institute, Liverpool John Moores University, Twelve Quays House, Egerton Wharf, Birkenhead CH41 1LD, UK \\ ${ }^{10}$ Department of Physics, Institute for Computational Cosmology, Durham University, South Road, Durham DH1 3LE, UK \\ 11 The Oskar Klein Centre for Cosmoparticle Physics, Department of Physics, Stockholm University, AlbaNova, SE-106 91 Stockholm, Sweden \\ ${ }^{12}$ Physics Department, University of California, Davis, CA 95616, USA \\ ${ }^{13}$ Institute of Geophysics and Planetary Physics, Lawrence Livermore National Laboratory, Livermore, CA 94551, USA \\ ${ }^{14}$ Centro de Astrofísica da Universidade do Porto, Rua das Estrelas, 4150-762 Porto, Portugal \\ ${ }^{15}$ Departamento de Física e Astronomia da Faculdade de Ciências da Universidade do Porto, Rua do Campo Alegre, 687, 4169-007 Porto, Portugal \\ Received 2011 June 23; accepted 2012 April 4; published 2012 May 21
}

\begin{abstract}
This paper presents both the result of a search for fossil systems (FSs) within the XMM Cluster Survey and the Sloan Digital Sky Survey and the results of a study of the stellar mass assembly and stellar populations of their fossil galaxies. In total, 17 groups and clusters are identified at $z<0.25$ with large magnitude gaps between the first and fourth brightest galaxies. All the information necessary to classify these systems as fossils is provided. For both groups and clusters, the total and fractional luminosity of the brightest galaxy is positively correlated with the magnitude gap. The brightest galaxies in FSs (called fossil galaxies) have stellar populations and star formation histories which are similar to normal brightest cluster galaxies (BCGs). However, at fixed group/cluster mass, the stellar masses of the fossil galaxies are larger compared to normal BCGs, a fact that holds true over a wide range of group/cluster masses. Moreover, the fossil galaxies are found to contain a significant fraction of the total optical luminosity of the group/cluster within $0.5 R_{200}$, as much as $85 \%$, compared to the non-fossils, which can have as little as $10 \%$. Our results suggest that FSs formed early and in the highest density regions of the universe and that fossil galaxies represent the end products of galaxy mergers in groups and clusters.
\end{abstract}

Key words: catalogs - galaxies: clusters: general - galaxies: evolution - galaxies: formation

Online-only material: color figures

\section{INTRODUCTION}

Hierarchical models of structure formation predict that galaxy groups and clusters (hereafter referred to collectively as galaxy systems) form via the gravitational infall of field galaxies and smaller systems (White \& Rees 1978). The effects of dark energy will eventually halt the infall (Nagamine \& Loeb 2003), at which time the system can be considered fully assembled. Dynamical friction will cause some of these galaxies to merge, resulting in the formation of a massive galaxy near the base of the gravitational potential (e.g., Barnes 1989; Dubinski 1998). A magnitude gap can then develop as the timescale for orbital decay by dynamical friction is inversely proportional to galaxy mass, and so the more massive galaxies will tend to merge first (D'Onghia et al. 2005; Dariush et al. 2010). The cooling timescale of the intergalactic medium is longer, so the system will be surrounded by a halo of X-ray-emitting gas. Such systems have come to be referred to as "fossil groups."

The most commonly accepted definition of a fossil group (Jones et al. 2003) requires (1) an $R$-band magnitude gap of two or greater between the two brightest galaxies located within half the virial radius of the system and (2) extended $\mathrm{X}$-ray emission with a bolometric X-ray luminosity of
$L_{X \text {,bol }} \gtrsim 5 \times 10^{41} h_{70}{ }^{-2} \mathrm{erg} \mathrm{s}^{-1}$. The X-ray criterion guarantees the existence of at least a group-size halo, while the optical criterion ensures (approximately) that there are no $L^{*}$ galaxies inside the radius for orbital decay by dynamical friction.

Originally, the term "fossil group" (or "fossil galaxy group") referred to an apparently isolated galaxy, which due to a surrounding halo of X-ray-emitting gas, was assumed to have previously resided in a group (Ponman et al. 1994). The word "fossil" was used because the merger history of the group was thought to be contained within this single galaxy. Since then, fossil groups (i.e., galaxies) have been found within actual galaxy groups and this has lead to a degree of confusion and a gradual shift from "fossil group" referring to the individual galaxy to the group as a whole. Additionally, since the definition of a fossil group that has developed only sets lower limits on both the size of the magnitude gap and the X-ray luminosity $\left(L_{X}\right)$, it is possible to find fossil groups with properties that are more consistent with those of galaxy clusters, as we do in this work (but see also Mendes de Oliveira et al. 2006, 2009; Khosroshahi et al. 2006b). Therefore, we replace the word "group" with the word "system," so that "fossil system" (FS) refers to a galaxy group or cluster that satisfies the above two criteria and "fossil galaxy" (FG) refers to the most-luminous galaxy in an FS. This 
is the nomenclature that we shall use throughout the remainder of this paper.

The initial interpretation of FGs was that they represent the end product of galaxy merging in groups or clusters. The magnitude gap in an FS was accounted for by an early formation epoch that allowed time for sufficient mergers. The observed regular, symmetric X-ray emission (Khosroshahi et al. 2007) and highly concentrated mass profiles (Jones et al. 2003; Khosroshahi et al. 2004) of FSs support this idea. Likewise, simulations have shown that FSs form a large fraction of their mass at high redshift, and that they form earlier than nonFSs (D'Onghia et al. 2005; Dariush et al. 2007; von BendaBeckmann et al. 2008). Additionally, D’Onghia et al. (2005) found a correlation between the magnitude gap at $z=0$ and the formation time of the FS in the sense that early-forming systems have larger gaps (see also Dariush et al. 2010). Note, however, that infall can result in a smaller magnitude gap without the system necessarily being late-forming.

Simulations by von Benda-Beckmann et al. (2008) suggest that the formation of FSs is primarily driven by the relatively early infall of massive satellites with the magnitude gap arising after the system has built up half of its final mass. The formation appears particularly efficient if the infall occurs along filaments with small impact parameters (D'Onghia et al. 2005; SommerLarsen 2006; von Benda-Beckmann et al. 2008).

Studies of the FS luminosity function (Mendes de Oliveira et al. 2006; Cypriano et al. 2006; Zibetti et al. 2009; Aguerri et al. 2011) have found parameters that are consistent with the universal luminosity function of clusters derived by Popesso et al. (2005), although they are deficient in $\sim L^{*}$ galaxies. It is possible that FSs formed with a deficit of $\sim L^{*}$ galaxies (Mulchaey $\&$ Zabludoff 1999). This alternate formation scenario interprets FSs as "failed groups" in which the majority of the available gas was initially used up in a single luminous galaxy rather than in several. Similarly, Proctor et al. (2011) found that FSs have low richnesses but masses comparable to those of clusters resulting in high dynamical mass-to-light ratios. As with their masses, FS $\mathrm{X}$-ray scaling relations are more consistent with clusters than groups (Mendes de Oliveira et al. 2006, 2009; Khosroshahi et al. $2006 \mathrm{~b}$ ), leading to the suggestion that FSs are merely representative of an evolutionary phase (von Benda-Beckmann et al. 2008) - after 4 Gyr, 90\% of FSs in simulations are found to have become non-fossil (Dariush et al. 2007).

In one of the only studies of its kind, Díaz-Giménez et al. (2008) looked at the merger history of FGs in simulated FSs within the Millennium Simulation Galaxy Catalogue. They find that, like brightest cluster galaxies (BCGs), FGs are mainly formed by gas-poor mergers but that FGs are formed later than BCGs, i.e., they undergo mergers at lower redshifts, despite the fact that FSs assembled most of their virial mass at higher redshifts in comparison with non-FSs. No age differences were found between the stellar populations in FSs and bright field ellipticals (La Barbera et al. 2009), which is consistent with a later formation if the mergers were gas-poor. Numerous studies have been made of the growth of BCGs and the results are conflicting. Simulations performed in a hierarchical context predict that BCGs are the result of multiple mergers and therefore should continue to grow until $z \sim 0.5$ (e.g., De Lucia $\&$ Blaizot 2007). Observationally, however, BCGs are found to evolve passively since $z \sim 1$ (Brough et al. 2002, 2007; Stott et al. 2008, 2010; Whiley et al. 2008; Collins et al. 2009).

Defining FS samples is difficult due to the need for both good quality X-ray and optical data, and so most of our understanding has come from simulations. The initial observational studies focused on small samples or single objects and so the number of known FSs remains small (see Mendes de Oliveira et al. 2006 for a good summary). Enabled by the large quantity of data produced by surveys such as the Sloan Digital Sky Survey (SDSS; York et al. 2000), more recent studies have focused on defining and analyzing larger samples of FSs (e.g., Santos et al. 2007; La Barbera et al. 2009; Voevodkin et al. 2010; Miller et al. 2012), but in most cases these samples lack the high-quality $\mathrm{X}$-ray data necessary to ensure that the definition of an FS is met, and often the criteria have been relaxed to such an extent that the possibility of substantial contamination is high.

The purpose of this study is two-fold. First, we want to take the high quality X-ray data from the XMM Cluster Survey (XCS; Romer et al. 2001; Lloyd-Davies et al. 2011; Mehrtens et al. 2011) and combine it with the optical data from the SDSS Data Release 7 (DR7) to produce a secure sample of FSs; we choose purity over quantity. Second, we want to compare the stellar mass assembly and the stellar populations of FGs to various other samples, and to examine the X-ray scaling relations of FSs.

The XCS is a serendipitous search for galaxy clusters using all publicly available data in the $X M M$ Science Archive. Its main aims are to measure cosmological parameters and trace the evolution of X-ray scaling relations. The first data release from XCS (XCS-DR1; Mehrtens et al. 2011, hereafter M11) contains 503 clusters, of which 402 have measured X-ray temperatures $\left(T_{X}\right)$ and luminosities. The serendipitous nature of XCS is a big advantage because it allows the detection of smaller systems, which have a higher probability of satisfying the optical criterion in the FS definition (Cui et al. 2011).

The layout of the remainder of the paper is as follows. We outline the samples used in Section 2, including a review of the definition of an FS and our methodology for searching for them. In Section 3, we describe the various data sets utilized in this study. The results of our study into the stellar masses and the stellar populations of fossil galaxies are presented in Section 4, while Section 5 presents the results of our study of the X-ray scaling relations and stellar mass assembly of FGs and BCGs. A discussion of all these results can be found in Section 6 and our conclusions are presented in Section 7.

We assume a $\Lambda$ CDM cosmology with a Hubble parameter of $H_{0}=70 \mathrm{~km} \mathrm{~s}^{-1} \mathrm{Mpc}^{-1}$, a dark matter density parameter of $\Omega_{\Lambda}=0.73$, and a matter density parameter of $\Omega_{M}=0.27$.

\section{SAMPLES}

In this section, we outline our methodology for searching for FSs within the XCS and the SDSS and present our final sample of 17. We also discuss two samples of BCGs, one optically selected and one X-ray selected, which we use as comparison samples. We begin by making a few comments on how FSs are defined.

\subsection{The Definition of a Fossil System}

The most commonly used definition of an FS is that found in Jones et al. (2003), which is reproduced in the introduction. This definition is motivated by physical arguments but is not beyond modification. The X-ray criterion was set to only select galaxies that reside in at least a group-sized halo and so does not have much scope for modification, while the adopted magnitude gap, on the other hand, does. 
This is because studies of the magnitude gap in both clusters (Milosavljević et al. 2006) and groups (Tavasoli et al. 2011) have shown a wide range of gaps. The average magnitude gap between a $0.1<z<0.2 \mathrm{BCG}$ and the second brightest cluster member is found to be 0.5 (Pipino et al. 2011, see also Loh $\&$ Strauss 2006), with poor systems exhibiting a larger average gap. Statistically, it is easier to get large magnitude gaps when the number of galaxies in the system is low (Dariush et al. 2007; Cui et al. 2011). In simulations, Dariush et al. (2007) found that the strongest X-ray FS candidates are those with the highest $\mathrm{X}$-ray luminosity as these systems are not expected to have a large luminosity gap entirely by chance.

Dariush et al. (2010) have suggested, based on numerical simulations, that a magnitude gap of 2.5 between the brightest and fourth-brightest galaxies is a better indicator of an FS. They find that the 2 mag gap is better at finding high-mass systems but the 2.5 mag gap finds $50 \%$ more early-forming systems and those that are in the fossil phase longer.

The definition of an FS that we adopt here is a combination of the X-ray criterion of Jones et al. (2003) and the optical criterion of Dariush et al. (2010). Therefore, for a system to be classified as a fossil by us it needs to have $L_{X} \gtrsim 5 \times 10^{41} h_{70}^{-2} \mathrm{erg} \mathrm{s}^{-1}$ and a magnitude gap of 2.5 in the $r$ band between the brightest and the fourth brightest galaxies located within half the virial radius, which we denote as $\Delta m_{14}$. The virial radius is approximated with $R_{200}$, the radius at which the average density is equal to 200 times the critical density of the universe, and $L_{X}$ is the bolometric $X$-ray luminosity inside this radius. In Appendix A, we investigate how our results change if the standard definition of an FS is adopted, i.e., replacing the Dariush et al. (2010) magnitude gap with that of Jones et al. (2003).

\subsection{The Fossil System Sample}

Previous searches for FSs began by looking for galaxies that satisfied the magnitude gap criterion and then tried to match them to an X-ray detection. Here, we take the opposite approach and examine the galaxies associated with an extended X-ray source to determine the magnitude gap.

We begin with the $Z_{o o}{ }^{\mathrm{DR} 7}$ and $\mathrm{Zoo}^{\mathrm{S} 82}$ candidate catalogs as described in M11. In addition, we also allow for $X M M$ PI-targeted cluster observations to be included in our final sample (such systems are noted in Table 1). Thus, our catalog of FSs contains serendipitously discovered FSs, as well as previously known groups/clusters which have been re-classified as FSs. Since we are studying the stellar populations of FGs, we also required that the brightest galaxy near the center of each candidate have a measured SDSS spectrum and we used the redshift of that galaxy to define the redshift of the system. These constraints complicate the FS selection, and so for this work we avoid making any conclusions which require a known selection function (e.g., constraining the number density of FSs).

We then identified FSs by examining the color-magnitude relation around each candidate. We used a system similar to the XCS-Zoo that is described in detail in M11, the main difference being the addition of color-magnitude diagrams (CMDs) to help with the estimation of the magnitude gap. Specifically, we visually examined X-ray images and optical color images from the SDSS overlaid with X-ray contours. These X-ray and optical images were created over $3^{\prime} \times 3^{\prime}, 6^{\prime} \times 6^{\prime}$, and $12^{\prime} \times 12^{\prime}$ fields of view. At the same time, we compared the images to the $r$ versus $r-i$ CMDs using SDSS DR7 imaging data.
A system was either designated an FS candidate or not based on the inspection of these images and CMDs. To be classified as an FS candidate the system must satisfy the following criteria.

1. Optical. A bright elliptical galaxy at or near the location of the X-ray source.

2. X-ray. Obviously an extended source, e.g., rather than a blend of point sources (see M11).

3. CMD. $\Delta m_{14}>2.0$ in the $3^{\prime} \times 3^{\prime}$ image.

It has recently been noted that the SDSS photometry systematically underestimates the luminosities of nearby BCGs (Bernardi et al. 2007; Lauer et al. 2007), with the discrepancy being a function of the BCG radius. This problem affects DR7, which we use, and is best described in the "Imaging Caveats" page of DR8. ${ }^{16}$ Therefore, we use a magnitude gap of only 2.0 when selecting the FS candidates, instead of the adopted gap of 2.5, to allow for the correction of this problem (von der Linden et al. 2007). All sources were examined by at least three coauthors and those that were classified as an FS candidate at least twice ( $\sim 60$ in total) were then examined more closely.

Defining magnitude gaps in clusters requires accurate measures of $R_{200}, L_{X}$, and the magnitudes of the galaxies, as well the ability to reject foreground/background galaxies. Therefore, the FS candidates that passed the above initial criteria were then put through the XCS analysis pipelines in order to measure $T_{X}$ and $L_{X}$ (see Section 3.1 for more details). After doing so, we re-calculated the CMDs using the estimated $R_{200}$ values (specifically, out to $\left.0.5 R_{200}\right)$. We discuss the robustness of our sample and results to changes to $R_{200}$ in Appendix A.

We also applied background corrections to the magnitudes of the large central galaxies using the algorithm defined in von der Linden et al. (2007) and tested in Voevodkin et al. (2008). The average size of this correction is 0.3 mag but could be as large as $1.0 \mathrm{mag}$. We note that many existing FS samples that rely on SDSS data (e.g., Santos et al. 2007; La Barbera et al. 2009; Miller et al. 2012) have not made this important correction. This would result in incorrect FG luminosities (which, as we shall see in Section 5, is a significant fraction of the total system luminosity), smaller magnitude gaps, and lower levels of completeness.

Only then were we able to measure the magnitude gap from the brightest central galaxy out to some physical distance. In estimating the magnitude gap, we only considered galaxies that were within \pm 0.2 from the $r-i$ color of the central galaxy. This is reasonably generous since the scatter in the red sequence is found to be less than half this amount. We also discarded galaxies from the CMDs that had SDSS spectroscopic redshifts $>2000 \mathrm{~km} \mathrm{~s}^{-1}$ away from the central galaxy. For those galaxies with a photometric redshift (Csabai et al. 2007), we discarded galaxies beyond $\Delta z_{\text {phot }}=0.1$. This cut is conservative, since the average photometric redshift error for galaxies with $m_{r} \sim 19.7$ is 0.04 . No difference in the number of systems classified as fossils was found when this photometric redshift cut was doubled to $\Delta z_{\text {phot }}=0.2$. This resulted in a clean (but possibly incomplete) sample of $17 \mathrm{FSs}$. This iterative procedure is important and it allowed us to rigorously check which of the 60 FS candidates were in fact true FSs.

The positions of these systems in the parameter space used to define FSs are shown in Figure 1. The red circles are our sample while the blue squares are the XCS-DR1 groups/clusters of M11 (see Section 2.3 for more details). The horizontal and

\footnotetext{
$\overline{16}$ http://www.sdss3.org/dr8/imaging/caveats.php
} 
Table 1

The Details of the Fossil Systems in our Sample

\begin{tabular}{|c|c|c|c|c|c|c|c|c|c|c|}
\hline$\overline{\mathrm{ID}}$ & XCS Name & Literature Name & $\Delta m_{12}$ & $\Delta m_{14}$ & $R_{200}$ & $T_{X}$ & $L_{X}$ & $L_{\text {tot }}$ & $\Sigma L_{24}$ & $\overline{\text { FS References }}$ \\
\hline 1 & XMMXCS J015315.0+010214.2 & WHL J015315.2+010220 & $1.7 \pm 0.02$ & $2.7 \pm 0.02$ & $0.664_{-0.008}^{+0.007}$ & $1.08_{-0.02}^{+0.02}$ & $0.05_{-0.03}^{+0.11}$ & $3.06_{-0.06}^{+0.06}$ & $0.57_{-0.01}^{+0.06}$ & - \\
\hline 2 & XMMXCS J030659.8+000824.9 & SDSS CE J046.719402+00.163919 & $1.3 \pm 0.06$ & $2.5 \pm 0.04$ & $1.01_{-0.18}^{+0.27}$ & $2.3_{-0.7}^{+1.3}$ & $0.014_{-0.010}^{+0.03}$ & $1.05_{-0.03}^{+0.06}$ & $0.29_{-0.01}^{+0.01}$ & - \\
\hline 3 & XMMXCS J073422.2+265143.9 & [DMM2008] IV & $2.4 \pm 0.30$ & $3.0 \pm 0.01(+0.5)$ & $0.67_{-0.05}^{+0.10}$ & $1.1_{-0.1}^{+0.2}$ & $0.21_{-0.19}^{+0.79}$ & $2.86_{-0.74}^{+0.74}$ & $0.41_{-0.21}^{+0.09}$ & 1 \\
\hline 4 & XMMXCS J083454.8+553420.9 & WHL J083454.9+553421 & $2.4 \pm 0.03$ & $3.0 \pm 0.03$ & $1.17_{-0.07}^{+0.06}$ & $3.9_{-0.4}^{+0.4}$ & $9.66_{-2.74}^{+2.75}$ & $12.47_{-0.29}^{+0.43}$ & $1.15_{-0.03}^{+0.29}$ & - \\
\hline 5 & XMMXCS J092540.0+362711.1 & NSC J092521+362758 & $1.9 \pm 0.02$ & $2.8 \pm 0.01(-0.3)$ & $1.14_{-0.09}^{+0.12}$ & $3.0_{-0.4}^{+0.6}$ & $1.03_{-0.87}^{+0.87}$ & $4.53_{-0.23}^{+0.23}$ & $0.61_{-0.01}^{+0.26}$ & - \\
\hline 6 & XMMXCS J101703.6+390250.7 & A0963 & $2.2 \pm 0.02$ & $2.7 \pm 0.02$ & $1.63_{-0.02}^{+0.02}$ & $6.6_{-0.1}^{+0.1}$ & $15.80_{-0.25}^{+0.29}$ & $25.50_{-0.81}^{+0.81}$ & $1.44_{-0.03}^{+0.81}$ & - \\
\hline 7 & XMMXCS J104044.4+395710.4 & A1068 & $2.3 \pm 0.02$ & $3.1 \pm 0.03$ & $1.217_{-0.006}^{+0.006}$ & $3.54_{-0.03}^{+0.03}$ & $8.39_{-0.16}^{+0.17}$ & $11.44_{-0.20}^{+0.20}$ & $2.00_{-0.03}^{+0.18}$ & - \\
\hline 8 & XMMXCS J123024.3+111127.8 & BLOX J1230.6+1113.3 ID & $2.1 \pm 0.18$ & $3.5 \pm 0.03$ & $0.54_{-0.01}^{+0.01}$ & $0.80_{-0.03}^{+0.03}$ & $0.018_{-0.002}^{+0.10}$ & $1.61_{-0.06}^{+0.20}$ & $0.39_{-0.03}^{+0.03}$ & - \\
\hline 9 & XMMXCS J123338.5+374114.9 & - & $2.6 \pm 0.01$ & $3.2 \pm 0.02$ & $0.58_{-0.04}^{+0.03}$ & $0.9_{-0.1}^{+0.1}$ & $0.03_{-0.01}^{+0.01}$ & $0.95_{-0.02}^{+0.02}$ & $0.142_{-0.003}^{+0.023}$ & - \\
\hline 10 & XMMXCS J124425.9+164758.0 & WHL J124425.4+164756 & $0.5 \pm 0.20$ & $2.3 \pm 0.10$ & $0.63_{-0.06}^{+0.08}$ & $1.3_{-0.2}^{+0.1}$ & $0.06_{-0.03}^{+0.05}$ & $5.85_{-0.38}^{+0.38}$ & $0.91_{-0.11}^{+0.38}$ & - \\
\hline 11 & XMMXCS J130749.6+292549.2 & ZwCl 1305.4+2941 & $2.6 \pm 0.18$ & $3.1 \pm 0.03$ & $1.04_{-0.03}^{+0.03}$ & $3.2_{-0.2}^{+0.2}$ & $1.94_{-0.11}^{+0.10}$ & $12.83_{-0.40}^{+0.40}$ & $1.12_{-0.03}^{+0.23}$ & - \\
\hline 12 & XMMXCS J131145.1+220206.0 & MaxBCG J197.94248+22.02702 & $2.1 \pm 0.06$ & $2.7 \pm 0.06$ & $1.16_{-0.06}^{+0.06}$ & $3.4_{-0.2}^{+0.2}$ & $1.57_{-0.39}^{+0.69}$ & $10.97_{-0.30}^{+0.29}$ & $1.39_{-0.03}^{+0.28}$ & - \\
\hline 13 & XMMXCS J134825.6+580015.8 & - & $2.0 \pm 0.02$ & $2.6 \pm 0.02$ & $0.78_{-0.05}^{+0.07}$ & $1.6_{-0.2}^{+0.2}$ & $0.08_{-0.04}^{+0.03}$ & $3.55_{-0.08}^{+0.08}$ & $0.37_{-0.01}^{+0.08}$ & - \\
\hline 14 & XMMXCS J141627.7+231525.9 & $\mathrm{ZwCl} 1413.9+2330$ & $1.8 \pm 0.02$ & $2.9 \pm 0.02$ & $1.25_{-0.06}^{+0.06}$ & $3.7_{-0.2}^{+0.2}$ & $1.42_{-0.51}^{+1.10}$ & $7.99_{-0.14}^{+0.14}$ & $1.08_{-0.02}^{+0.11}$ & $2,3,4,5,6,7$ \\
\hline 15 & XMMXCS J141657.5+231239.2 & - & $2.8 \pm 0.02$ & $3.1 \pm 0.01$ & $0.56_{-0.03}^{+0.04}$ & $0.9_{-0.1}^{+0.1}$ & $0.017_{-0.004}^{-0.005}$ & $1.51_{-0.04}^{+0.04}$ & $0.171_{-0.004}^{+0.035}$ & - \\
\hline 16 & XMMXCS J160129.8+083856.3 & - & $2.4 \pm 0.03$ & $3.1 \pm 0.03(-0.3)$ & $0.77_{-0.04}^{+0.05}$ & $1.7_{-0.2}^{+0.2}$ & $0.84_{-0.35}^{+0.35}$ & $2.77_{-0.09}^{+0.09}$ & $0.45_{-0.07}^{+0.27}$ & - \\
\hline 17 & XMMXCS J172010.0+263724.7 & SDSS-C4 3072 & $1.9 \pm 0.03$ & $2.4 \pm 0.03$ & $1.54_{-0.01}^{+0.01}$ & $5.53_{-0.04}^{+0.04}$ & $21.58_{-7.85}^{+23.45}$ & $18.64_{-1.26}^{+1.26}$ & $1.94_{-0.04}^{+1.26}$ & 8 \\
\hline
\end{tabular}

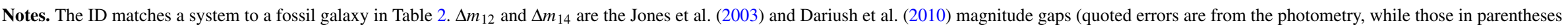

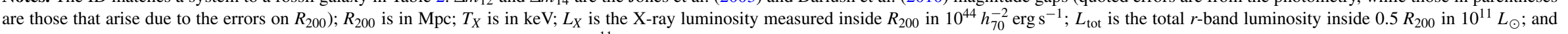
$\Sigma L_{24}$ is the total $r$-band luminosity of the second to fourth brightest galaxies in $10^{11} L_{\odot}$.

${ }^{a}$ The system was an $X M M$ target.

${ }^{b}$ The magnitude of the FG has not been corrected, so the magnitude gap of this system is a lower limit; see Appendix B.10 for details

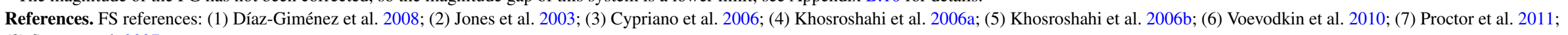
(8) Santos et al. 2007. 


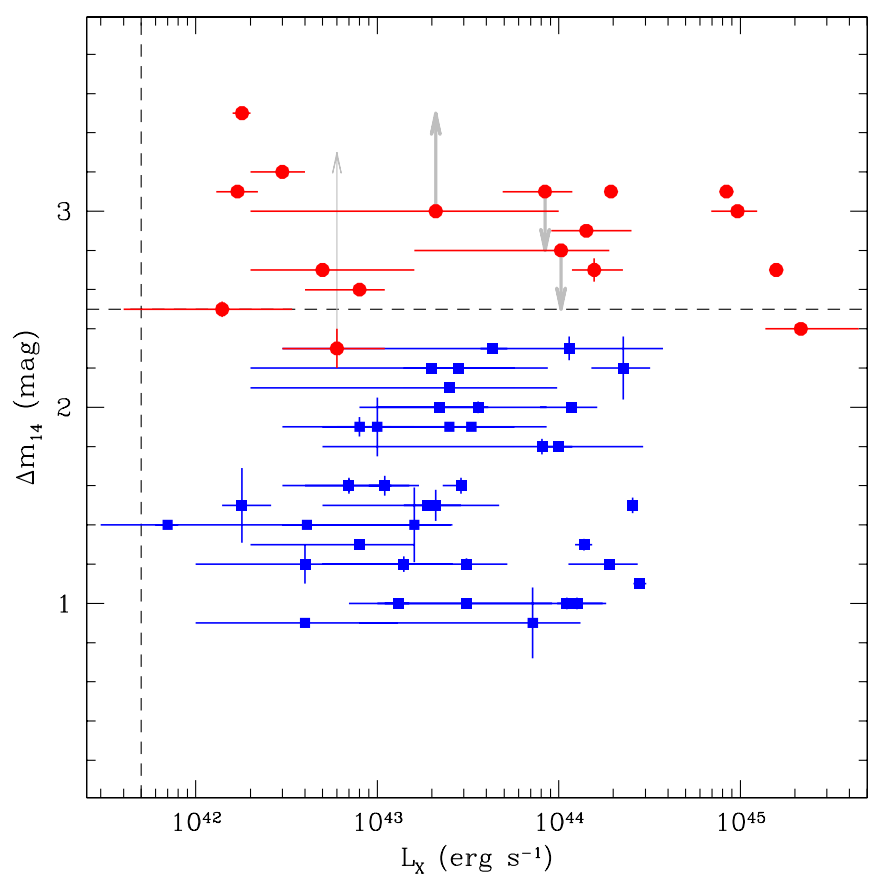

Figure 1. Distribution of our sample (red circles) and the XCS groups/clusters (blues squares) in the parameter space used to classify FSs. Points that lie above and to the right of the dashed lines are classified as FSs. Two systems that have $\Delta m_{12}<2.5$ are classified as FSs. The system with the arrow is XMMXCS $\mathrm{J} 124425.9+164758.0$, which has a highly uncertain sky-subtraction correction that we elect not to apply. If we apply the correction (of $1.0 \mathrm{mag}$ ), then this results in a magnitude gap of 3.3, as denoted by the thin arrow. The other system is XMMXCS J172010.0+263724.7, which has a magnitude gap of $\Delta m_{14}=2.45$ and is close enough to 2.5 that we accept it as an FS. The three thick arrows indicate how the magnitude gap changes when the error on $R_{200}$ is taken into account. For all other systems the magnitude gap is unaffected by the errors on $R_{200}$.

(A color version of this figure is available in the online journal.)

vertical dashed lines denote the optical and X-ray criterion, respectively; points that lie above and to the right of these lines are classified as FSs. There are two systems that have $\Delta m_{14}<2.5$, which we accept as FSs. The system with the arrow is XMMXCS J124425.9+164758.0 (hereafter referred to simply as J124425.9), which has an FG with a double nucleus. This makes the sky-subtraction correction highly uncertain and so we elect not to apply it. If we were to apply the estimated correction of $1.0 \mathrm{mag}$, then $\Delta m_{14}$ changes from 2.3 to 3.3 , which is denoted by the thin arrow in Figure 1. Even if we only apply the average sky-subtraction correction of the other 16 FSs (0.3 mag), then $\Delta m_{14}=2.6$ and so we accept J124425.9 as an FS. The other system is XMMXCS J172010.0+263724.7, which actually has a magnitude gap of $\Delta m_{14}=2.45$ and is close enough to 2.5 that we accept it as an FS too. Our results do not change if these two FSs are excluded. We note that four FSs were targeted for observation by XMM and are thus not a part of the XCS-DR1, which includes only serendipitously discovered clusters. These targeted systems are labeled in Table 1 and our conclusions are unaffected if we exclude them from our analyses.

For three FSs, there are galaxies near $0.5 R_{200}$, such that a slightly smaller $R_{200}$ excludes one or more of them as members or a slightly larger $R_{200}$ includes one or more of them as members. If the galaxy is among the four brightest in the system, then this can have the effect of increasing or decreasing the magnitude gap, respectively. We show the magnitude of this systematic effect in Figure 1 (thick arrows) after changing $R_{200}$ by one standard deviation of its statistical error (see Section 3.4).
The magnitude gaps for the other 14 FSs are unaffected by the statistical uncertainties on $R_{200}$.

Details of the FSs are given in Table 1 and details of the FGs are given in Table 2. Descriptions of the individual systems along with CMDs and SDSS images with XCS contours overlaid are given in Appendix B.

\subsection{The BCG Samples}

One of the main aims of this study is to characterize the stellar populations and the stellar mass assembly of FGs and to compare them with those of BCGs in non-FSs. For this purpose, we use two samples of BCGs, one optically selected and one $\mathrm{X}$-ray selected. For a fuller description of the various catalogs on which the samples were based, the reader is referred to the references below.

The first sample of BCGs consists of optically selected galaxies drawn from two catalogs: the C4 BCG catalog (von der Linden et al. 2007) and the maxBCG catalog (Koester et al. 2007). We use both these catalogs because, combined, they fully cover the redshift range of our FGs. The second sample of BCGs is drawn from the XCS (M11). We will refer to the former sample as the optical BCGs, the latter as the XCS BCGs, and, when combined, simply as BCGs. There are considerably less XCS BCGs than optical BCGs, but they are drawn from an X-ray-selected cluster sample and therefore are useful in assessing any bias that may result from comparing an optically selected sample (the optical BCGs) to an X-ray-selected sample (the FGs).

The von der Linden et al. (2007) C4 catalog is based on the Miller et al. (2005) C4 catalog but utilizes a better algorithm for identifying the BCG. It is derived using the SDSS DR4 spectroscopic sample and it identifies clusters in position, redshift, and color parameter space, assuming that a fraction of the cluster galaxies form a red sequence. The $\mathrm{C} 4$ catalog contains 625 BCGs at $z<0.1$.

The maxBCG algorithm for cluster detection relies on two characteristics of galaxy clusters: the brightest galaxies in a cluster occupy a narrow region of color-magnitude space (the red sequence) and the brightest galaxy in the cluster is located near the center of the galaxy distribution. Galaxies are designated as BCGs based on the product of two likelihoods. The first is the likelihood that the galaxy is spatially located in an overdensity of galaxies with similar $g-r$ and $r-i$ colors. The second is the likelihood that it has the color and magnitude properties typical of BCGs. The algorithm can be run on any catalog of galaxies and doing so on the SDSS database results in a catalog consisting of 13,823 BCGs with redshifts of $0.1<z<0.3$, which is over $85 \%$ complete for halos with masses above $1 \times 10^{14} h^{-1} M_{\odot}$. To match our FS sample, we restrict the maxBCG catalog to $z \leqslant 0.25$.

XCS-DR1 (M11) consists of 503 optically confirmed $\mathrm{X}$-ray clusters at $z<1.46,402$ of which have published X-ray temperatures and luminosities. From these, we draw a sample of clusters that are within the SDSS footprint, have an observed spectrum, and have $z<0.25$ (the redshift of our highest-redshift FS), for use as a comparison sample. For each cluster, a BCG was identified from an SDSS image and in the cases where the BCG was not obvious the brightest galaxy closest to the X-ray position was selected. The positions of these clusters in the parameter space used to define FSs are shown in Figure 1. Note in defining the magnitude gap for the XCS BCGs we used the same method that was used in defining the FS magnitude gaps. 
Table 2

The Details of the Fossil Galaxies in Our Sample

\begin{tabular}{|c|c|c|c|c|c|c|c|}
\hline ID & SDSS Name & $L_{\text {gal }}$ & $z$ & $M_{*}$ & Age & $Z$ & $\log ($ SSFR $)$ \\
\hline 1 & J015315.24+010220.6 & $1.81 \pm 0.06$ & 0.0597 & 0.47 & 10.4 & 0.030 & -12.23 \\
\hline 2 & J030658.71+000833.2 & $0.69 \pm 0.03$ & 0.0751 & 0.29 & 10.5 & 0.030 & -12.20 \\
\hline 3 & $\mathrm{~J} 073422.21+265144.9$ & $1.88 \pm 0.05$ & 0.0796 & 0.82 & 8.1 & 0.032 & -12.37 \\
\hline 4 & J083454.90+553421.1 & $6.69 \pm 0.28$ & 0.2412 & $\ldots$ & $\ldots$ & $\ldots$ & $\ldots$ \\
\hline 5 & J092539.05+362705.5 & $2.24 \pm 0.06$ & 0.1121 & 0.75 & 11.3 & 0.030 & -12.31 \\
\hline 6 & $\mathrm{~J} 101703.63+390249.4$ & $7.08 \pm 0.21$ & 0.2056 & 1.56 & 10.8 & 0.031 & -12.70 \\
\hline 7 & $\mathrm{~J} 104044.49+395711.2$ & $4.95 \pm 0.17$ & 0.1381 & $\ldots$ & $\ldots$ & $\ldots$ & $\ldots$ \\
\hline 8 & $\mathrm{~J} 123024.67+111122.8$ & $1.27 \pm 0.05$ & 0.1169 & 0.37 & 10.9 & 0.021 & -11.96 \\
\hline 9 & $\mathrm{~J} 123337.74+374122.0$ & $0.81 \pm 0.02$ & 0.1023 & 0.33 & 11.8 & 0.026 & -12.30 \\
\hline 10 & $\mathrm{~J} 124425.43+164756.9$ & $3.64 \pm 0.35$ & 0.2346 & 0.52 & 11.8 & 0.027 & -12.29 \\
\hline 11 & J130749.23+292548.2 & $6.71 \pm 0.22$ & 0.2406 & 1.36 & 10.9 & 0.028 & -12.13 \\
\hline 12 & $\mathrm{~J} 131146.19+220137.2$ & $4.36 \pm 0.26$ & 0.1715 & 0.97 & 9.5 & 0.027 & -12.13 \\
\hline 13 & $\mathrm{~J} 134825.78+580018.7$ & $2.35 \pm 0.07$ & 0.1274 & 0.59 & 8.5 & 0.033 & -11.77 \\
\hline 14 & $\mathrm{~J} 141627.37+231522.5$ & $3.53 \pm 0.10$ & 0.1382 & 1.08 & 9.9 & 0.032 & -12.29 \\
\hline 15 & $\mathrm{~J} 141657.46+231242.5$ & $1.27 \pm 0.03$ & 0.1159 & 0.42 & 8.7 & 0.033 & -11.26 \\
\hline 16 & $\mathrm{~J} 160129.75+083850.6$ & $1.71 \pm 0.06$ & 0.1875 & 1.02 & 11.3 & 0.028 & -12.50 \\
\hline 17 & $\mathrm{~J} 172010.04+263732.0$ & $5.40 \pm 0.18$ & 0.1596 & 0.92 & 5.6 & 0.031 & -11.44 \\
\hline
\end{tabular}

Notes. The ID matches a galaxy to a system in Table $1 . L_{\text {gal }}$ is the luminosity of the FG in $10^{11} L_{\odot}, M_{*}$ is the stellar mass of the FG in $10^{12} M_{\odot}$, the age of the FG is in Gyr, $Z$ is the metallicity of the FG as a percentage, and SSFR is the specific star formation rate in yr ${ }^{-1}$. Luminosities and $z$ are taken from the SDSS; $M_{*}$, age, and $Z$ are from STARLIGHT; and SSFR are taken from the MPA-JHU database.

There are $39 \mathrm{XCS}$ clusters that satisfy the above three criteria and these clusters have a flat distribution in redshift space very similar to that of the FSs. The optical BCGs have a distribution that peaks at $z \sim 0.25$; therefore, to minimize any evolutionary effects, we randomly resampled the original sample of 14448 BCGs (C4 and maxBCG combined) to create a sample that has a flat distribution over the same redshift range as our FSs, resulting in a final sample of 2687 optical BCGs.

\section{DATA}

In this section, we describe the various data sets (X-ray, optical, and stellar population parameters) that were used in this study. We conclude the section with a discussion of the errors on the various quantities.

\subsection{X-Ray Data}

The X-ray $\left(T_{X}\right.$ and $\left.L_{X}\right)$ and $R_{200}$ data used in this paper come from the XCS-DR1 (M11). The XCS is a serendipitous search for galaxy clusters using all publicly available data in the $X M M$ Science Archive (Romer et al. 2001). The procedures used for measuring $T_{X}$ and $L_{X}$, and for estimating $R_{200}$ are outlined in Section 4 of Lloyd-Davies et al. (2011, hereafter LD11), ${ }^{17}$ so we give only a brief description here. $T_{X}$ was measured by performing spectral fitting to background-subtracted spectra with XSPEC. The best fit was determined using the maximum likelihood Cash statistic (Cash 1979). $L_{X}$ was measured by extrapolating surface brightness fits to a simple one-dimensional, spherically symmetric, $\beta$-profile model (Cavaliere \& Fusco-Femiano 1976). $R_{200}$ is then estimated from the Arnaud et al. (2005) $T_{X}-R_{200}$ relation.

\subsection{Optical Data}

All galaxy magnitudes are SDSS DR7 Petrosian magnitudes that have been extinction corrected, $k$-corrected (kcorrect v4.1.4; Blanton \& Roweis 2007), and sky-subtraction corrected (von der Linden et al. 2007). Total optical luminosities of

\footnotetext{
17 Actually, the process for estimating $L_{X, 500}$ and $R_{500}$ is described, but it is similar to that for estimating $L_{X, 200}$ and $R_{200}$.
}

groups/clusters $\left(L_{\text {tot }}\right)$ are estimated from all galaxies that are: (1) less than 0.2 redder and 0.2 bluer than the $r-i$ color of the BCG and (2) dimmer than the BCG and within a radius of $0.5 R_{200}$ to an absolute $r$-band magnitude of -19.67 (or 20.8 at $z=0.25$ ). Galaxies with spectra are restricted to those within $2000 \mathrm{~km} \mathrm{~s}^{-1}$ of the cluster, while those without are restricted to $\Delta z_{\text {phot }}=0.1$, where we use the $z_{\text {phot }}$ from the SDSS DR7. These selection criteria are identical to those used in defining the magnitude gaps of the systems (see Section 2.2). The radius of $0.5 R_{200}$ was chosen as it matches the radius used to define an FS and changing it to $R_{200}$ has no effect on the results. In addition to the optical data, spectroscopic redshifts $(z)$ of the FGs/BCGs are also obtained from the SDSS and used to define the redshift of the system.

\subsection{Stellar Population Data}

The SDSS has provided galaxy spectra in unprecedented numbers, which has allowed for statistical studies of the star formation histories and stellar populations of various galaxy samples to be performed. The by-products of these efforts have been numerous new methods for estimating the associated parameters, such as ages, metallicities, and elemental abundances, etc. Commonly, these data are stored in public databases for general use or the method is made public to allow it to be applied to different samples.

Each of these methods approach what is essentially the same task in a variety of ways. Thus, each method has it own set of strengths and weaknesses. In this study, we utilize two different methods/databases: STARLIGHT (Cid Fernandes et al. 2005) and MPA-JHU (Kauffmann et al. 2003; Brinchmann et al. 2004; Tremonti et al. 2004; Salim et al. 2007). Brief outlines of both are given below, but for more details readers are referred to the references provided. We obtain stellar masses $\left(M_{*}\right)$, ages, and metallicities $(Z)$ from STARLIGHT and specific star formation rates (SSFRs) from MPA-JHU, which also provides stellar masses that we use to test the robustness of some of our results. We chose to use the STARLIGHT data over the MPA-JHU data for two reasons: the diffusion map method of selecting a population 
basis used in Richards et al. (2009) has been shown to reduce the age/metallicity degeneracy; and we note that the MPA-JHU released neither ages nor metallicities beyond DR4, which is a much smaller sample than DR7.

\subsubsection{STARLIGHT}

The population synthesis code STARLIGHT (Cid Fernandes et al. 2004, 2005) fits an observed spectrum with a linear combination of single theoretical stellar populations (coeval and chemically homogeneous) computed with the Bruzual \& Charlot (2003) evolutionary synthesis models. Richards et al. (2009) show that the accuracy of this technique is highly dependent on the choice of input basis of simple stellar population (SSP) spectra. Thus, we use the Richards et al. (2009) diffusion map methods to choose appropriate bases of prototype SSP spectra. These methods allow us to approximate the continuous grid of age and metallicity of SSPs of which galaxies are truly composed. As shown in detail in Richards et al. (2009), we obtain robust stellar ages, stellar metallicities, and stellar masses and at the same time significantly reduce the degeneracy between age and metallicity. We correct the stellar masses for the fact that they are observed within a $3^{\prime \prime}$ diameter fiber using the same correction as applied to the MPA-JHU stellar masses (see Section 3.3.2). In Table 2, we provide the results of these fits for stellar mass, luminosity-weighted ages, and stellar mass-weighted metallicities.

\subsubsection{MPA-JHU}

The MPA-JHU team has publicly released catalogs of derived physical properties for a sample of SDSS galaxies, but the only property that we make use of is the SSFR (Brinchmann et al. 2004), although we make use of their stellar masses to estimate an average error for the STARLIGHT stellar masses. The catalog is being recreated for the SDSS DR7, for which there are properties for 818,333 galaxies.

The method of Brinchmann et al. (2004) to estimate star formation rates is built on the methodology of Charlot et al. (2002) and the modeling of emission lines by Charlot \& Longhetti (2001), which combine the Bruzual \& Charlot (1993) galaxy evolution models with the emission line modeling from CLOUDY (Ferland 1996). Dust attenuation is the major source of uncertainty in estimating star formation rates, and Brinchmann et al. (2004) follow the dust treatment outlined in Charlot \& Fall (2000), making an initial estimate of dust attenuation based on $\mathrm{H} \alpha / \mathrm{H} \beta$. A grid of model $\mathrm{H} \alpha$ line strengths (with a particular dust attenuation applied) is compared with the observed spectrum and the best match selected using a Bayesian approach similar to Kauffmann et al. (2003). H $\alpha$ luminosity is then converted to an SSFR following Charlot \& Longhetti (2001). The estimated SSFR of each FG is given in Table 2.

Stellar masses are estimated by multiplying the dust-corrected luminosity of the galaxy by the stellar mass-to-light ratio predicted by their best-fit model using the Bayesian approach mentioned above (Kauffmann et al. 2003). They extrapolate the mass-to-light ratio and the dust attenuation values estimated within the SDSS fiber to obtain total stellar masses.

\subsection{Errors}

Derivation of the X-ray data errors is described in LD11 and we will only give a summary here. For $L_{X}$ and $T_{X}$, the parameter is stepped (both in the negative and positive directions) from its best-fit value until the fit statistic increases by the amount required for the confidence region needed (i.e., 68\%). The $R_{200}$ errors were calculated by propagating the $T_{X}$ errors through the Arnaud et al. (2005) $T_{X}-R_{200}$ relation.

Errors on $L_{\text {gal }}, L_{\text {tot }}, \Sigma_{24}$, and the magnitude gaps are from the SDSS photometry and when converting to solar luminosities both the error on the absolute $g$-band magnitude $(0.02 \mathrm{mag})$ and the solar $g-r$ color $(0.02 \mathrm{mag}$; Bilir et al. 2005) are propagated through. In addition to these statistical errors, we also derive errors on $\Delta m_{14}$ due to the errors on $R_{200}$. This only affects the magnitude gap in 3 out of the 17 FSs and in none of the 3 does it negate the system's classification as an FS. These systems have this error provided in parentheses after the statistical error in Table 1 . In some cases, the errors on $R_{200}$ also induce additional errors on the total optical luminosities. In half of our sample, the error on $R_{200}$ does not add or subtract any galaxies into the total optical luminosity. In the other half, the luminosities do change and these have the largest errors in Table 2. These systematic luminosity errors are still small when compared to the total optical luminosity of the system which is dominated by a single very bright galaxy. However, the fractional error on the $\Sigma_{24}$ can be quite large and one-sided.

There are no errors on age and metallicity because STARLIGHT does not provide any. MPA-JHU does provide errors on their SSFR but we do not use them since, as for age and metallicity, we are comparing the SSFR distributions and not individual systems. We estimate average stellar mass error from the scatter in the differences between the STARLIGHT and MPA-JHU stellar mass estimates, which encodes the scatter introduced by the use of two distinct statistical techniques and two different SSP models. We use these average errors $(0.047$ dex for the FSs and 0.079 dex for the XCS BCGs) in relevant plots but do not provide them in Table 2.

\section{STELLAR POPULATIONS}

If FGs formed at a different epoch to BCGs or followed a different evolutionary path, then evidence of this may be found within their stellar populations. In this section, we use the stellar masses, ages, and metallicities from STARLIGHT, and the SSFRs from the MPA-JHU database to compare the stellar populations of the FGs with those of the BCGs and investigate this issue.

We start by simply comparing the stellar masses of the three samples, the distributions of which are shown in Figure 2. The dashed lines mark the median mass for each of the samples. From this plot it can be clearly seen that FGs are among the most massive galaxies in the universe as, on average, they are more massive than both samples of BCGs. In fact, $\sim 80 \%$ of the FGs are more massive than the average BCG. The median stellar mass of the FGs is $\left\langle M_{*}\right\rangle=(7.6 \pm 1.2) \times 10^{11} M_{\odot}$, while optical BCGs have $\left\langle M_{*}\right\rangle=(4.1 \pm 1.9) \times 10^{11} M_{\odot}$ and XCS BCGs have $\left\langle M_{*}\right\rangle=(3.7 \pm 0.1) \times 10^{11} M_{\odot}$, i.e., FGs are roughly twice as massive as BCGs. We note that the same results are found when the MPA-JHU photometric-based stellar masses are substituted for the STARLIGHT spectroscopically based stellar masses.

Is this difference between the FG and the BCG stellar masses significant? A simple statistic to use would be the Students $t$-test, however, this test compares the samples individually and assumes that the stellar masses are drawn from a normal distribution. The Welch variation of the $t$-test is applicable if the sample variances are unequal or the sample sizes are different. If the distributions are not normal but they are similar (homoscedastic), then the Mann-Whitney $U$-test is appropriate for individual comparisons. Unfortunately, our data are not homoscedastic and we need to make multiple tests 


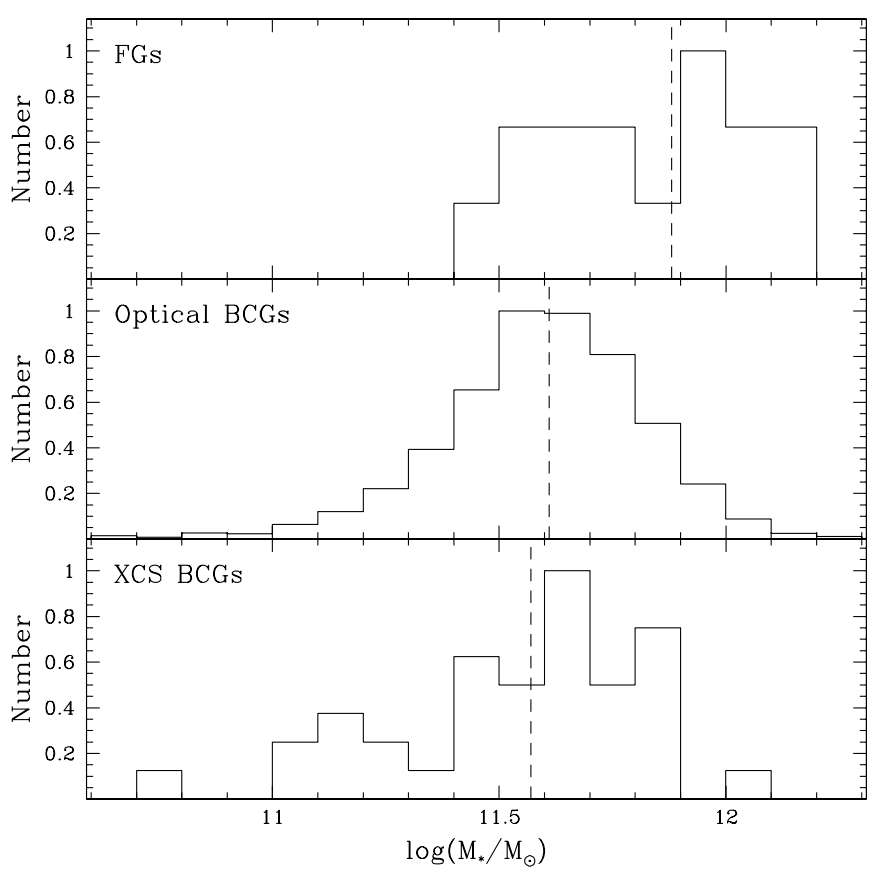

Figure 2. STARLIGHT stellar mass distributions for the various samples showing that FGs are among the most massive galaxies in the universe. The dashed lines mark the median mass for each of the samples.

simultaneously, therefore none of these standard techniques are desirable.

We are interested in a joint comparison of the masses from distributions which are not Gaussian and from samples of widely varying sizes (see Figure 2). Thus, we use multiple hypothesis testing (or simultaneous inference, see Shaffer 1995 for an excellent review on this subject), since if not accounted for, the multiplicity can result in an overestimation of statistical significance. Since we are comparing samples that have different sizes and distributions (i.e., the random variables are not heteroscedastic), the $t$-test family is not appropriate. Instead, we take the most robust statistical approach and control the family-wise error rates for all pairwise comparisons of group differences via the max $t$-test using a heteroscedastic consistent covariance estimation (see Herberich et al. 2010; Richardson 2011). On a technical note, we utilize single-step procedures to adjust the $p$-values for their multiplicity, meaning that the order of the tests is not important.

We find that the stellar masses of the FGs are significantly higher than those of the optical BCGs and the XCS BCGs. The probability that the FGs and the optical (XCS) BCGs have the same stellar masses is $<0.005(0.003)$ at the $95 \%$ confidence level. We also find that there is no difference between the stellar masses of the XCS and optical BCGs. We note again that these statistics are robust, in that no assumptions regarding the distribution, sample sizes, or variance homogeneity have been made. Had we not accounted for the heteroscedasticity of the samples, our reported probabilities would have been more than 10 times smaller (and our significance much higher). Had we used the Mann-Whitney $U$-test (which assumes that the distributions of the masses are the same in the different sets), we would have also reported 10 times smaller probabilities (and higher significances).

Performing the same statistical test on the stellar population parameters as the stellar masses, we find that there are no differences between any of the three samples in their distributions
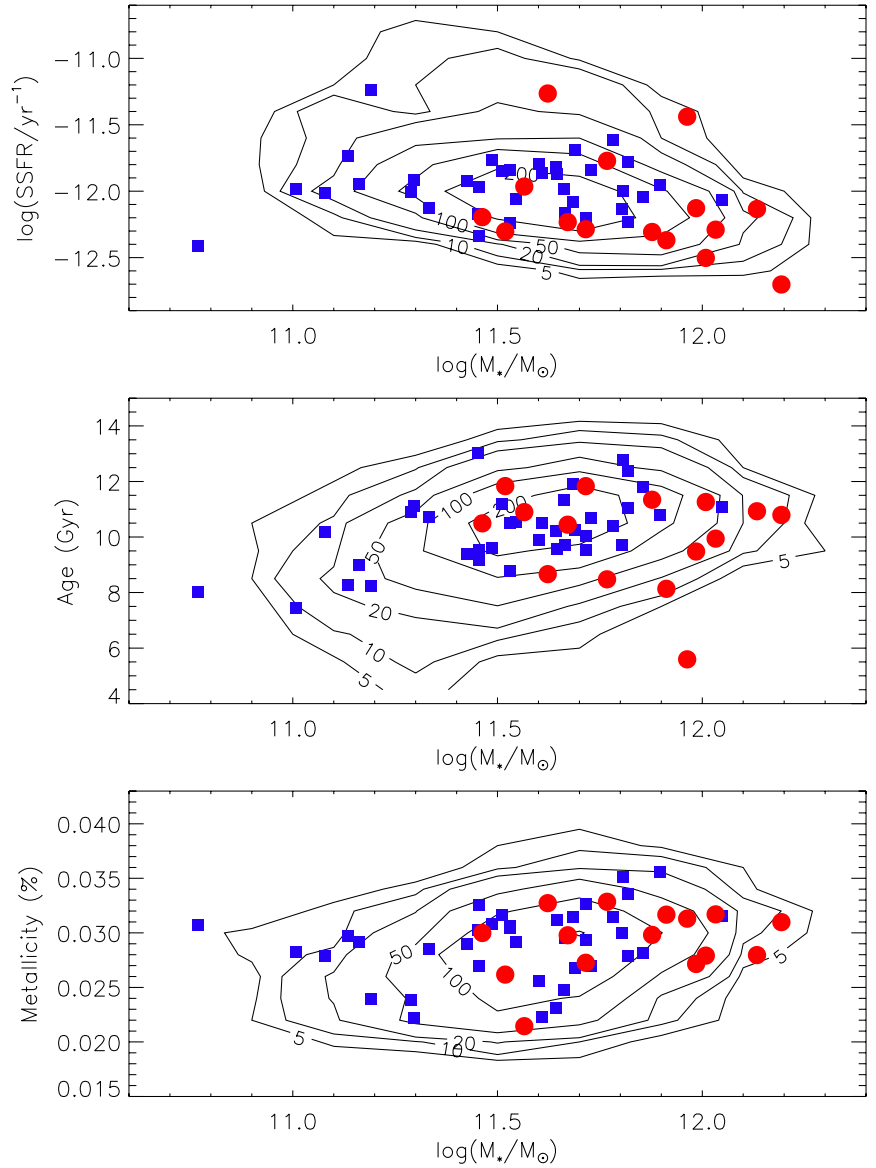

Figure 3. Scaling relations of SSFR (top), age (middle), and metallicity (bottom). The red circles are the FGs, the blue squares are the XCS BCGs, and the density contours are for the optical BCGs. The density contour levels are 5, 10, 20,50, 100, and 200 galaxies per bin. The stellar populations of the FGs are found to be consistent with those of the BCGs.

(A color version of this figure is available in the online journal.)

of SSFRs, ages, and metallicities, results which are confirmed by K-S testing. Similar results were found by La Barbera et al. (2009), although this was when comparing FGs to a sample of bright field ellipticals. No differences were reported between the ages and metallicities of both these samples.

We show the distribution of the three stellar population parameters as a function of stellar mass for all three samples in Figure 3, where red circles are the FGs, the blue squares are the XCS BCGs, and the density contours are for the optical BCGs. This is for the sake of clarity, since there are $\sim 2700$ optical BCGs. The density contour levels are 5, 10, 20, 50, 100, and 200 galaxies per bin. The fact that FGs are among the most massive galaxies in the universe is highlighted by these distributions where they mostly hug the massive edge of the BCG distributions.

Using a two-dimensional, two-sample $\mathrm{K}-\mathrm{S}$ test to compare the optical BCGs to the XCS BCGs (i.e., X-ray selected), we find no difference between any of the stellar population parameters, which allows us to compare the X-ray-selected FGs to the optically selected BCGs and take advantage of their larger sample size. Comparing the stellar populations in FGs with this sample, we find that the probability that their SSFRs and ages were drawn from the same distribution is $<0.005$ and $<0.01$ for their metallicities. Given that one-dimensional test above found no differences between the stellar population parameters of all 


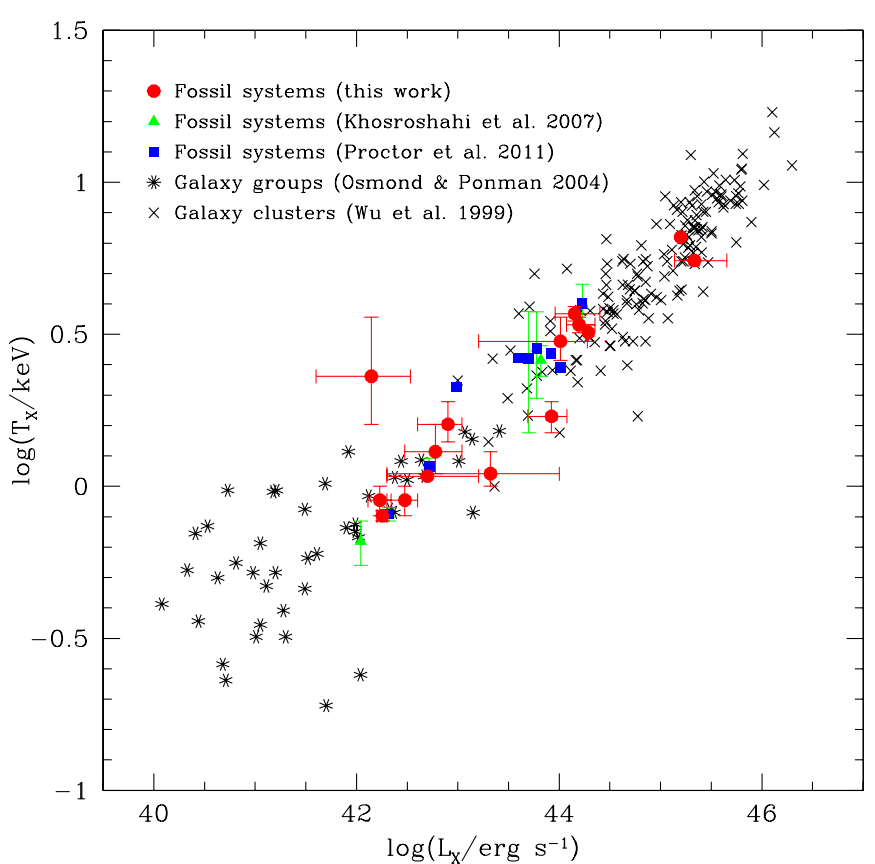

Figure 4. $L_{X}-T_{X}$ relation for the FSs (red circles). Also plotted are the FS data from Khosroshahi et al. (2007, green triangles) and Proctor et al. (2011, blue squares), the Wu et al. (1999) cluster data (black crosses), and the Osmond \& Ponman (2004) group data (black stars). FSs are found at all $L_{X}$ and $T_{X}$ except those of the most massive clusters. The absence of FSs at low $L_{X}$ and $T_{X}$ is due to the $L_{X}$ cutoff used in the definition of an FS.

(A color version of this figure is available in the online journal.)

three samples, we suggest that this confirms the earlier result that, on average, FGs are significantly more massive than BCGs and that the differences found in the two-dimensional testing are driven by these differences in mass.

In summary, we have performed a careful statistical analysis of the differences between the FG stellar masses and stellar populations, and those of the BCGs. Our analysis takes into account the differences in the sample distributions and sizes, as well as the multiplicity of the tests. We find that the FGs have significantly higher stellar masses than the BCGs but similar stellar populations. In the following sections, we explore the cause of this stellar mass growth in the FG with respect to the magnitude gap in the FS.

\section{STELLAR MASS ASSEMBLY}

We begin this section by looking at the X-ray scaling relations of the FSs and comparing them to those of the XCS BCGs. In Figure 4, we show the $L_{X}-T_{X}$ relation of the FSs (red circles). Also plotted are the FSs from Khosroshahi et al. (2007, green triangles) and Proctor et al. (2011, blue squares), the galaxy group data from Osmond \& Ponman (2004, black stars), and the galaxy cluster data from Wu et al. (1999, black crosses). From this figure, we see that FSs fall on the same relation that both galaxy groups and clusters do. In fact, they are found at all $L_{X}$, ranging over three orders of magnitude, except those of the most massive clusters. There is an absence of FSs at low $L_{X}$, which is due to a selection effect, i.e., the $L_{X}$ cutoff used in the definition of an FS. The lowest- $L_{X}$ FS, which has too large a $T_{X}$ for its $L_{X}$, is XMMXCS J030659.8+000824.9 (hereafter J030659.8) and we note that its classification as an FS is uncertain for reasons given in Appendix B.

In Figure 5, we examine the optical luminosity of the galaxy systems within $0.5 R_{200}\left(L_{\text {tot }}\right)$ as a function of $L_{X}$. Red circles

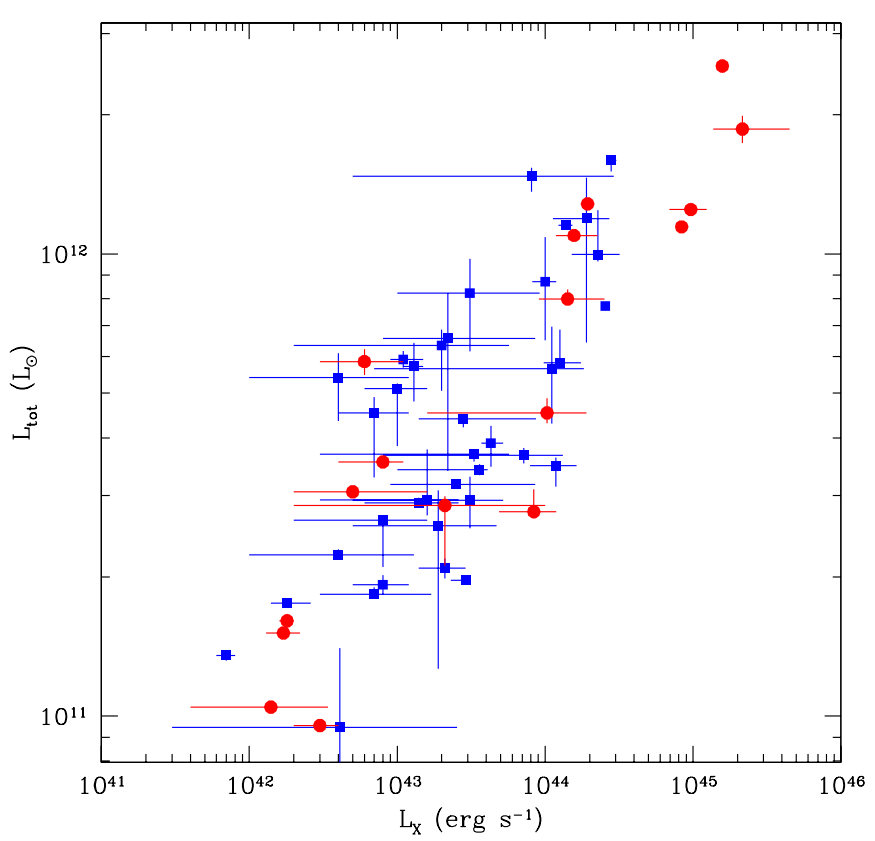

Figure 5. Optical luminosity of the galaxy system within $0.5 R_{200}$ as a function of $L_{X}$. There is no difference between the FS relation and that of the XCS clusters. Red circles are FSs and blue squares are XCS clusters.

(A color version of this figure is available in the online journal.)

are FSs and blue squares are XCS clusters. In a comprehensive study of the X-ray scaling relations of FSs by Khosroshahi et al. (2007), the authors found evidence that FSs were boosted in $L_{X}$ for a given optical luminosity (see also Santos et al. 2007), which is also predicted by the $N$-body simulations of D'Onghia et al. (2005). However, other studies have found no difference (Aguerri et al. 2011) and have concluded that there is a possible systematic difference between the studies or that the difference is real but only for less massive systems. We find no evidence for boosted $L_{X}$ in our data, rather we find that FSs, over more than three orders of magnitude in $L_{X}$, populate this diagram in the same way that non-FSs do.

The optical luminosity of the FSs is strongly correlated with $\mathrm{X}$-ray luminosity $\left(r_{S}=0.92, p<1 e-7\right)$ and the intrinsic scatter in $\log \left(L_{\text {tot }}\right)$ is only $0.14 \pm 0.02$ dex. Similar results are found for the XCS clusters, however, the intrinsic scatter is slightly larger. Substituting $T_{X}$ for $L_{X}$ (Figure 6) produces an even tighter relation, the intrinsic scatter in $\log \left(L_{\text {tot }}\right)$ is reduced to $0.10 \pm 0.01 \mathrm{dex}$, although the correlation is slightly weakened $\left(r_{S}=0.85, p<0.0001\right)$ due to a larger number of outliers. Again, similar results are found for the XCS clusters.

The X-ray temperatures are used to infer the radii within which we calculate the total optical luminosity of the systems. So it is reasonable to expect there to be some induced correlation between $T_{X}$ and $L_{\text {tot }}$. For example, as we scatter upward in $\mathrm{X}$-ray temperature, we would of course infer a larger radius to include the optical light from member galaxies (but never less). We tested the amplitude of this correlated scatter by examining the change in the fraction of light as we change $R_{200}$ based on the $T_{X}$ errors $( \pm 1 \sigma)$. We find that half of the FSs have optical luminosities that are identical after changing the radius. The other half have scatter in their $L_{\text {tot }}$ that correlates with scatter in $T_{X}$. These eight systems have the largest $L_{\text {tot }}$ errors in Table 2 . However, the slope of the fit to the fractional scatter in $L_{\text {tot }}$ versus $T_{X}$ in these systems is only 0.3 . For a given system, if the $T_{X}$ scatters up by $100 \%$, then the optical luminosity scatters 


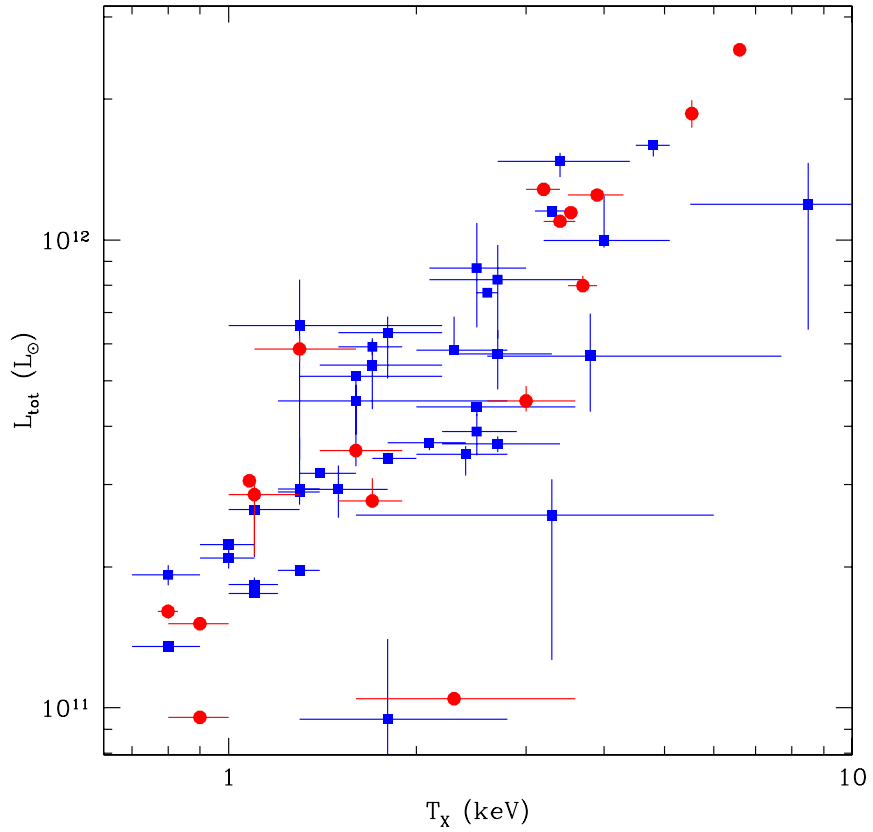

Figure 6. Optical luminosity of the galaxy system within $0.5 R_{200}$ as a function of $T_{X}$. The intrinsic scatter about the FS relation is greatly reduced both when compared to the FS relation with $L_{X}$ and the XCS cluster relations with $L_{X}$ and $T_{X}$. Red circles are FSs and blue squares are XCS clusters.

(A color version of this figure is available in the online journal.)

up by $\sim 30 \%$. In Figure 6, we see that the relationship between total optical luminosity and X-ray temperature is much steeper than this correlated scatter. For this reason and the fact that half the FS have uncorrelated scatter between $T_{X}$ and $L_{\text {tot }}$, we conclude that the real scatter in Figure 6 is not much larger than our measured scatter (see also Appendix A).

In Figure 7, we show the stellar mass of the dominant galaxy (i.e., an FG in an FS or a BCG in a non-FS) as a function of $T_{X}$. The red circles are our FGs, the blue squares are the $z \leqslant 0.25$ XCS BCGs, and the green triangles are the $z \sim 1$ BCGs from Stott et al. (2010). While the fits to the two BCG samples are consistent with each other (the green dashed and blue dotted lines), the fit to the FGs is offset to higher mass for a given $T_{X}$ (the red solid line). In Figure 8 , we present the same information as in Figure 7, except we represent the stellar mass of the dominant galaxy by its optical luminosity and the system mass by the total optical luminosity (see Section 3.3). Since we do not have stellar masses for all our FGs, nor for our BCGs, we make use of the $r$-band luminosity, which we expect to be a good proxy for stellar mass, to maximize our sample sizes. Just as in Figure 7, the FGs sample the upper edge of the BCG distribution and similar to Figures 5 and 6 , the scatter in the FGs is remarkably small.

We find that there is a strong correlation between $T_{X}$ and $M_{*}$ $\left(r_{S}=0.76, p<0.001\right)$ but that none of the stellar population parameters are found to be correlated. These results show that, while the mass of the system hosting the FG determines how massive it can become, it has little effect on the formation and evolution of the stars that compose it. Similar results are found when substituting $T_{X}$ with $L_{X}$ and the significance of the result is unaffected after excluding the two systems with $\Delta m_{14}<2.5$.

There are two results in Figure 7. The first is that FGs are more massive than XCS BCGs for any given $T_{X}$, and so the mean stellar mass of the ensemble of FGs has to be higher than non-FGs. This explains our results in Figure 2 and in Section 4,

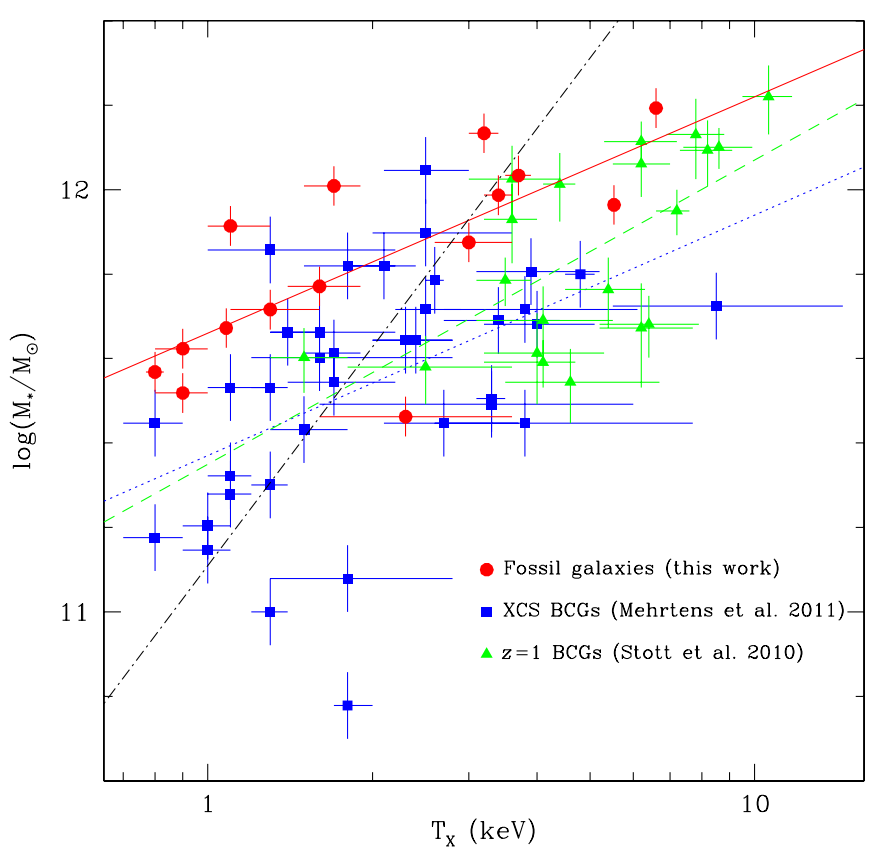

Figure 7. Variation of the stellar mass of the dominant galaxy with $T_{X}$ showing that, for a given $T_{X}$, FGs are among the most massive galaxies in the universe. The red circles are the FGs, the blue squares are the XCS BCGs, and the green triangles are the $z \sim 1$ BCGs of Stott et al. (2010). The red solid line is the fit to the FG data, the blue dotted line is the fit to the XCS BCGs, and the dashed green line is the fit to the Stott et al. (2010) BCG data. The black dot-dashed line shows the slope (1.72) of the power-law relation between $M_{500}$ and $T_{X}$ from Stott et al. (2010), which has been normalized to the FG fit at $T_{X}=3.0 \mathrm{keV}$.

(A color version of this figure is available in the online journal.)

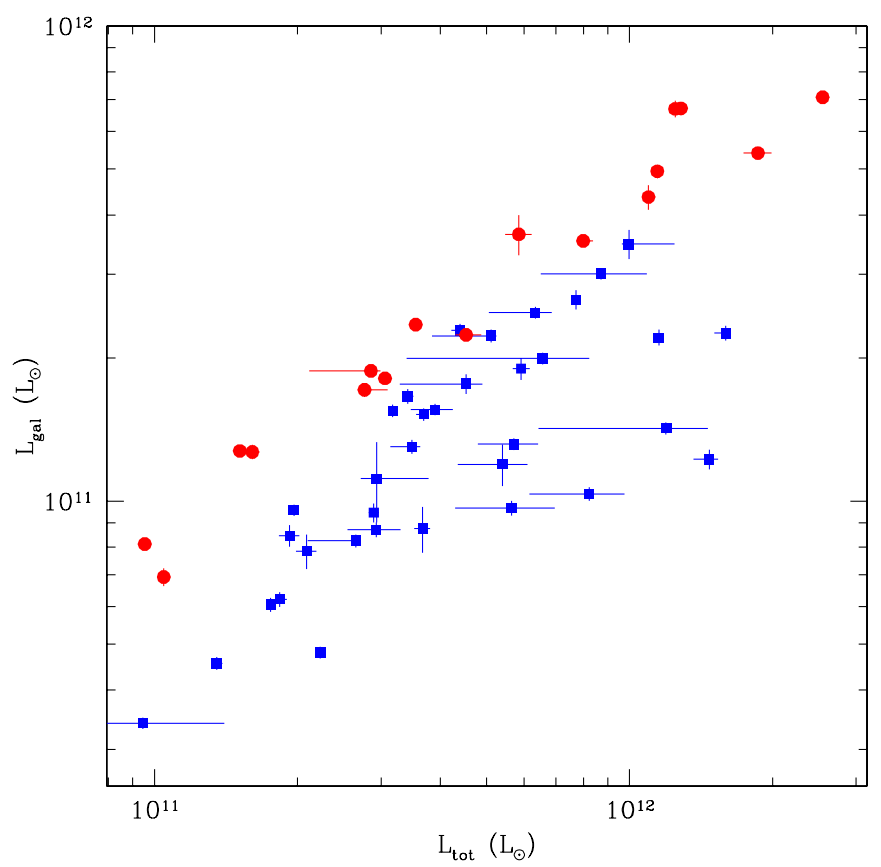

Figure 8. Same as Figure 7, but now we show the variation of the optical luminosity of the dominant galaxy with the total optical luminosity of the system. As in Figure 7, the FGs lie at the upper edge of the BCG distribution. Red circles are FGs and blue squares are BCGs.

(A color version of this figure is available in the online journal.)

where we found that the mean stellar mass of the FG sample is significantly higher than the non-FS samples.

Figure 7 shows that, at any given $T_{X}$, FGs have the highest stellar mass. There are, however, a few XCS BCGs that have large stellar masses and are not classified as fossils. We note 


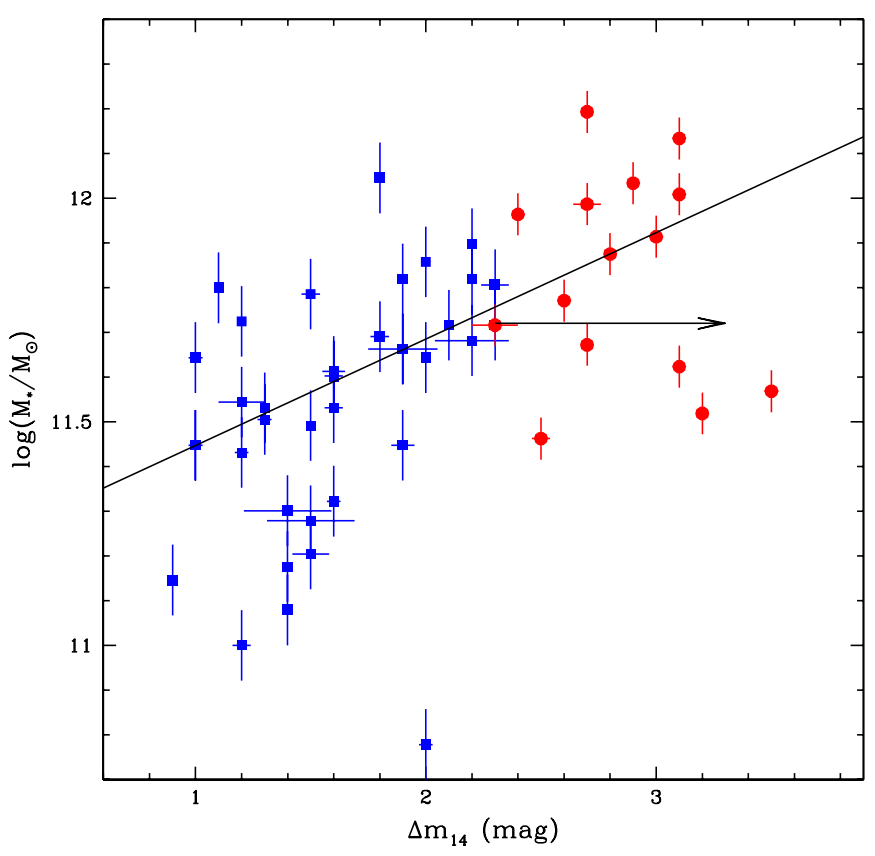

Figure 9. Highly significant correlation between $\Delta m_{14}$ and the stellar mass of the dominant galaxy. Red circles are FGs and blue squares are XCS BCGs. The solid line is the fit to the data, both the FGs and the XCS BCGs. The data point with the arrow is J124425.9 (see the text for details).

(A color version of this figure is available in the online journal.)

that these exceptions all have $\Delta m_{14}$ of $\sim 2$, which is close to the FS classification threshold. There is one FG that lies well below the rest, but we have reason to doubt the X-ray emission in this system and we refer the reader to Appendix B. 2 for a discussion.

The second result from Figure 7 is that the $z \sim 0.1$ FGs are $\sim 100 \%$ ( $\sim 40 \%)$ more massive than the $z \sim 1$ BCGs at $1 \mathrm{keV}$ $(10 \mathrm{keV})$. The fits to the low- and high-redshift BCG samples are consistent with each other. Therefore, if the $z \sim 1$ BCGs are the progenitors of the XCS BCGs, which are at $z \leqslant 0.25$, then this figure shows that BCGs do not have any significant growth at $z \lesssim 1$, consistent with previous studies (e.g., Stott et al. 2010). However, if BCGs from either sample are the progenitors of FGs, then the BCGs need to grow by $\sim 40 \%-100 \%$ to reach the average mass of an FG at $z \sim 0.1$. Semi-analytic models of early-type galaxy formation in clusters (e.g., De Lucia et al. 2006) suggest that FGs form via gas-poor mergers even at low redshifts (Díaz-Giménez et al. 2008), despite the fact that FSs assemble most of their virial mass at higher redshifts. There is some observational evidence that suggests BCGs continue to grow through dissipationless mergers even today (Brough et al. 2005, 2011) and simulations predict that a large fraction of the BCG mass today was accrued in the last 5 Gyr (De Lucia \& Blaizot 2007; Ruszkowski \& Springel 2009). Our results suggest that neither the high- nor low-redshift BCGs have evolved and that neither are the progenitors of FGs, unless significant major merging events by galaxies well outside the systems have yet to occur (e.g., Brough et al. 2011).

The black dashed line shows how the mass of the underlying system (i.e., the group or cluster mass) scales with temperature via the $M-T_{X}$ relation (core-excised) from Table 1 in Maughan (2007). Both the stellar masses of the dominant galaxies and the total masses of the groups/clusters scale with $T_{X}$, but their relationships are decoupled.

We can see from Figure 9 that the mass of the dominant galaxy is correlated with the size of the magnitude gap. The question is whether the magnitude gap and the stellar mass of

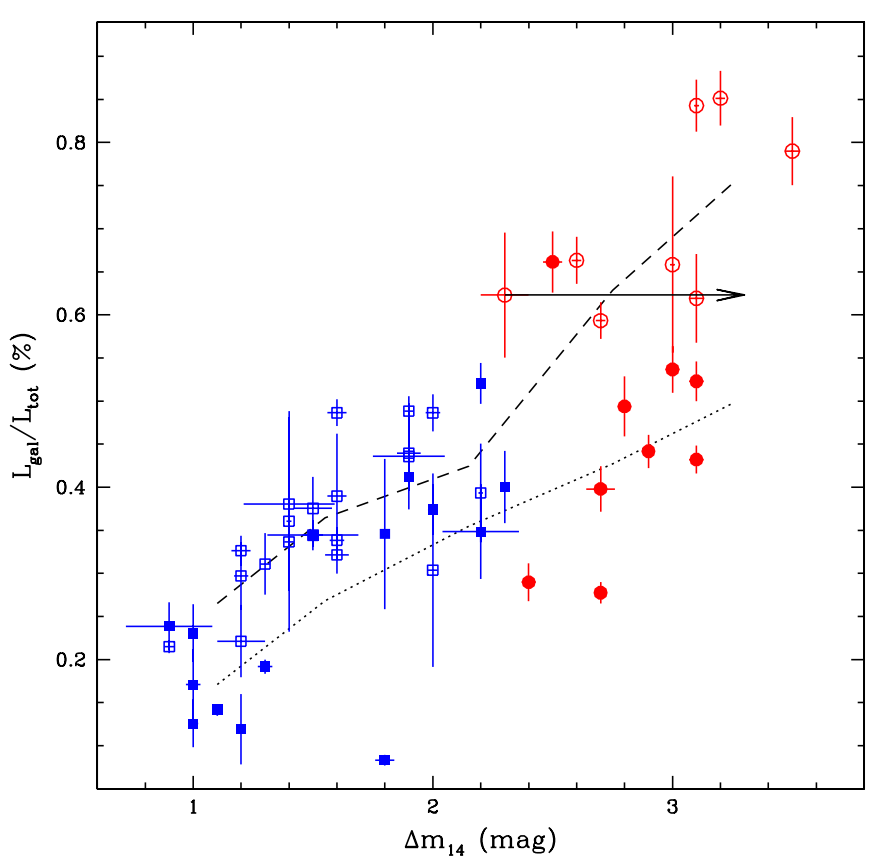

Figure 10. Fraction of total cluster light contained within the dominant galaxy as a function of $\Delta m_{14}$. The FGs can contain a significant fraction of the total light of a system (up to 85\%), while for the XCS BCGs this fraction is generally lower. Red circles are FGs and blue squares are XCS BCGs. The dashed line shows the median fraction for low-mass systems (open symbols; $T_{X}<2 \mathrm{keV}$ ) and the dotted line is for high-mass systems (filled symbols; $T_{X}>2 \mathrm{keV}$ ). The data point with the arrow is J124425.9 (see the text for details).

(A color version of this figure is available in the online journal.)

the galaxy are part of a cause and effect relationship: are the FG stellar masses large because the magnitude gap is large? These two quantities are found to be positively correlated with high significance $\left(r_{S}=0.58, p<0.00001\right)$. The solid line is the fit to the data, both the FGs and the XCS BCGs. The data point with the arrow in this plot (and the following plots) is J124425.9. The FG in this system has a double nucleus and so we elect not to apply the sky-subtraction correction; the arrow denotes the position of this system in this plot if we did apply the correction.

In Figure 10, we examine the fraction of total optical luminosity contained within the dominant galaxy as a function of the magnitude gap $\Delta m_{14}$. Red circles are FGs and blue squares are XCS BCGs. The trends seen in this figure are still present after we split the sample into high-mass systems (with $T_{X}>2 \mathrm{keV}$-dashed line and open symbols) and low-mass systems $\left(T_{X}<2 \mathrm{keV}\right.$ - dotted line and filled symbols). The magnitude gap bins, within which the medians were calculated, were chosen to contain approximately equal numbers and are as follows: 0.9-1.29, 1.3-1.79, 1.8-2.49, 2.5-2.99, and 3.0-3.5. The data point with the arrow is J124425.9 and it is not used to calculate the median in this or any following plots.

The fraction of total optical luminosity and the magnitude gap are highly correlated $\left(r_{S}=0.80, p=0\right.$ (actually $\left.2 e-12\right)$ ). There is a large spread in the fraction of light contained within the dominant galaxy, especially for the FGs. The values range from $30 \%$ all the way up to $85 \%$, consistent with the values that have been reported in the literature (Aguerri et al. 2011 found a value of $15 \%$ and Jones et al. 2000 a value of $70 \%$ ). This spread is caused by the strong correlation that exists between the dominant galaxy light fraction and the mass of the system (Lin \& Mohr 2004), which we show in Figure 11 using $T_{X}$ as a proxy for system mass, and the lack of a correlation between $\Delta m_{14}$ and system mass. In this figure, red circles are FGs and 


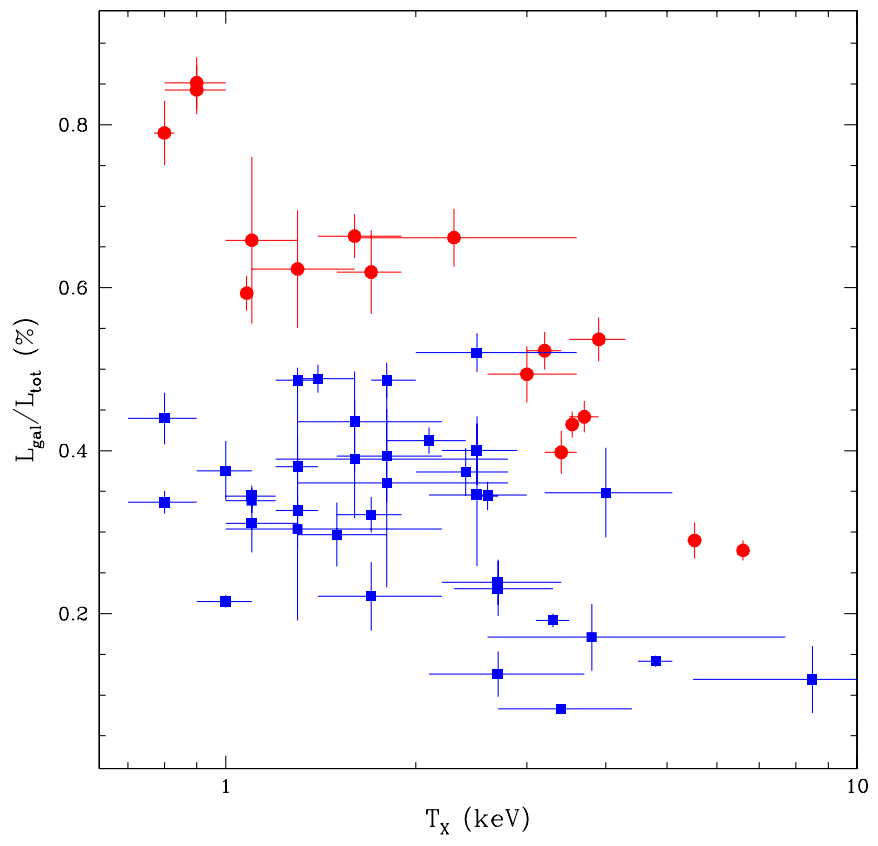

Figure 11. Fraction of the total optical luminosity in the system contained within the dominant galaxy as a function of $T_{X}$. Again, the FGs lie at the upper edge of the BCG distribution and form a tight sequence. The red circles are the FGs and the blue squares are the XCS BCGs.

(A color version of this figure is available in the online journal.)

blue squares are XCS BCGs. Similar to Figures 7 and 8, the FGs sample the upper edge of the BCG distribution and have a much smaller scatter.

Looking at the trends with system mass in Figure 10, it appears that there is a bi-modality at high magnitude gaps with a cleaner separation between the high-mass and low-mass systems. FGs in low-mass systems contain a larger fraction of the total optical luminosity of the system compared to the FGs in low-mass systems. However, our data are insufficient to be able to make any definite claims. The high-mass outlier is J030659.8, which was also an outlier in Figures 4 and 7.

In Figure 12, we show how the optical luminosity of the dominant galaxy changes with magnitude gap and in Figure 13 we show how the total optical luminosity of the second-to-fourth brightest galaxies $\left(\Sigma L_{24}\right)$ changes with magnitude gap. The symbols and line styles are the same as those used in Figure 10. From Figure 12, we see that the overall trend is for the luminosity of the dominant galaxy to increase as the magnitude gap grows. These two quantities are significantly correlated $\left(r_{S}=0.52\right.$, $p<0.00005)$. Similar to Figure 10, we see the suggestion of a bi-modality at high magnitude gaps. In Figure $13, \Sigma L_{24}$ is either constant or slightly decreasing, suggesting that very little merging (and certainly no major merging) is occurring among these galaxies. As in Figure 10, the high-mass outlier is J030659.8.

\section{DISCUSSION}

The debate about what FSs truly represent is far from over. Whether they are the end-point of mergers in groups/clusters or not is a contentious issue. If FSs do not represent a distinct class of objects, then the cause of their magnitude gap, which is their distinguishing feature, needs to be addressed. Two of the leading possibilities are an early formation epoch coupled with a favorable configuration for the rapid and efficient accretion of companions (Jones et al. 2003; Khosroshahi et al. 2004;

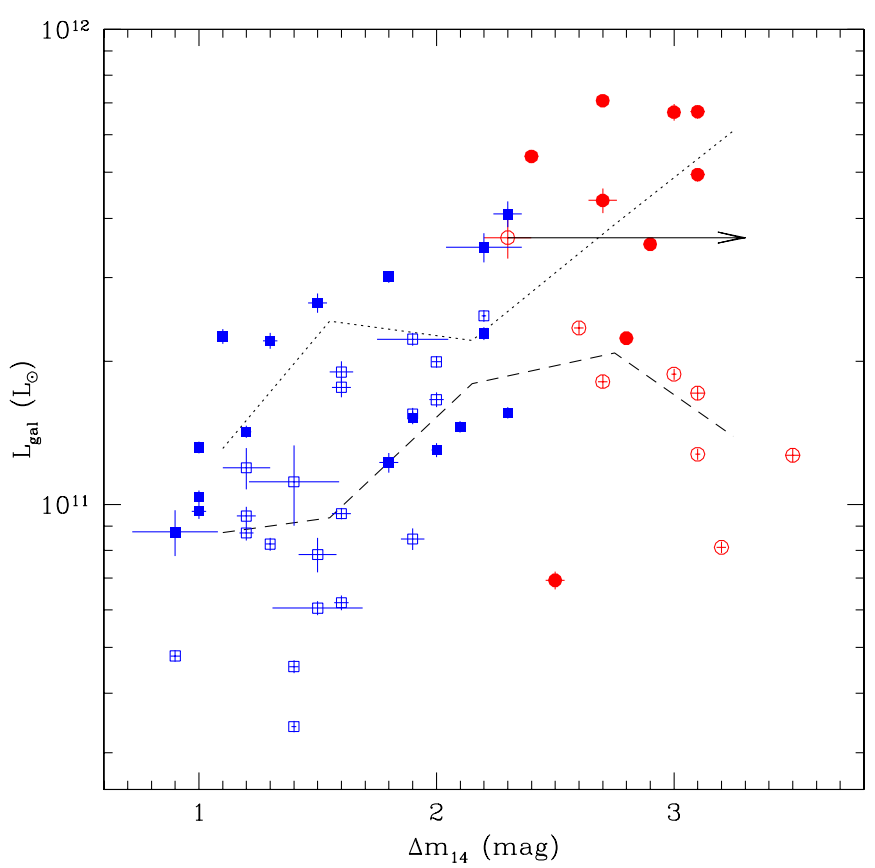

Figure 12. Dominant galaxy luminosity as a function of $\Delta m_{14}$. These plots show that luminous dominant galaxies are likely to be found in systems with large magnitude gaps. The red circles are the FGs and the blue squares are the XCS BCGs. The dashed line and the dotted line are the medians of the low-mass and high-mass systems. The data point with the arrow is J124425.9 (see the text for details).

(A color version of this figure is available in the online journal.)

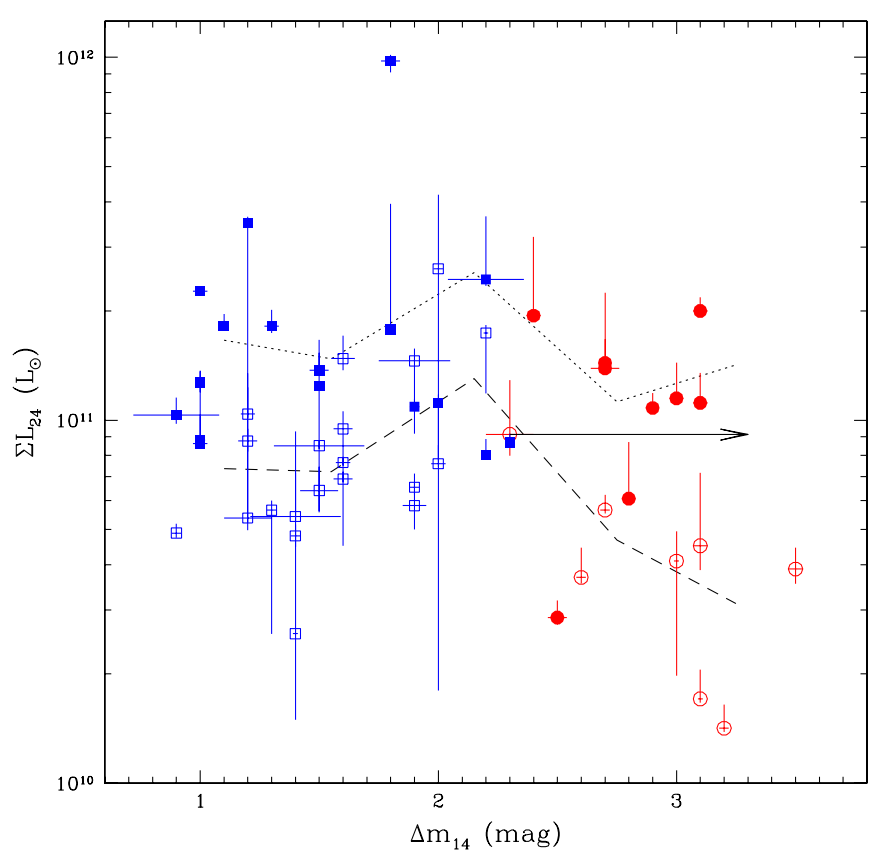

Figure 13. $\Sigma L_{24}$ as a function of $\Delta m_{14}$. The light contained within $\Sigma L_{24}$ remains constant or decreases as the magnitude gap increases. The red circles are the FGs and the blue squares are the XCS BCGs. The dashed line and the dotted line are the medians of the low-mass and high-mass systems. The data point with the arrow is $\mathrm{J} 124425.9$ (see the text for details).

(A color version of this figure is available in the online journal.)

D'Onghia et al. 2005; Khosroshahi et al. 2007; Dariush et al. 2007; von Benda-Beckmann et al. 2008) and the failed group scenario (Mulchaey \& Zabludoff 1999).

The results of this study provide some answers to this question. The observed facts that: (1) our FSs exist at all system 
masses; (2) our FSs are not deficient in $L^{*}$ galaxies (see the CMDs in Appendix B); and (3) the FGs have the largest stellar mass of any cluster BCG (as a function of the system mass), all rule out the failed group scenario. These are normal groups and clusters with populated red sequences but with an extremely massive BCG.

The correlations with magnitude gap (Figures 9, 10, 12, and 13) support the idea that the gap is an indicator of the evolutionary phase of an FS, with a large gap indicative of an early-forming system that contains an FG that has undergone many small mergers. This observational result is entirely consistent with the simulations of D'Onghia et al. (2005). Therefore, any model of FS formation and evolution must do the following as the magnitude gap grows.

1. The luminosity of the FG must increase.

2. The fraction of the total optical luminosity of the system contained in the FG must increase.

3. The total fraction of light contained in the second-to-fourth brightest galaxies must not increase.

If repeated mergers within the system are responsible for the above points, then there are two likely scenarios and the fact that the luminosity contained within the second-tofourth brightest galaxies stays constant with magnitude gap is important. First, the FG may grow by accreting many smaller galaxies, leaving the second-to-fourth brightest galaxies untouched. This scenario de-emphasizes dynamical friction and favors fast and efficient mergers with galaxies with low impact parameters. It is supported by the fact that in all our FSs only two (J030659.8 and J123338.5) have fourth brightest galaxies that are less luminous than $L^{*}$. In all other systems, the FG is so luminous that it is more than 2.5 mag brighter than $L^{*}$. Second, the FG could undergo fewer mergers with smaller galaxies and then develop the magnitude gap in a single equal-mass merger. This requires that the second-to-fifth brightest galaxies be of similar luminosity, so that when one is accreted the luminosity contained within the second-to-fourth brightest galaxies remains roughly constant. Both of these scenarios imply that the merging is more efficient in FSs than non-FSs, i.e., a higher fraction of the stellar mass is retained in the FG.

For the cluster-sized FSs, there is an additional formation scenario. The FG could form in a group where the relative velocities are conducive to merging, before accreting (or being accreted by) another system. This second system, however, must be lacking in bright galaxies, otherwise the magnitude gap would vanish and the system would no longer qualify as an FS, making this mode of formation unlikely, yet not impossible. Schirmer et al. (2010) found J0454-0309 to consist of two systems, a sparse cluster and an infalling FS, the latter they believe will seed the FG. They also find, outside a radius of $1.5 \mathrm{Mpc}$, two filaments that extend over $4 \mathrm{Mpc}$. In this study, we find two systems (J141627.7 and J141657.5) that are possibly interacting and represent a possible example of this scenario.

If only one process leads to the development of an FS, then it must be possible for this process to occur in a system of any mass; i.e., we have FSs ranging in mass from groups to clusters. Looking at Figures 10, 12, and 13, the case could be made for two different formation mechanisms: one in the high-mass systems and another in the low-mass systems. Although our data are not definitive, we will nonetheless speculate about this possibility.

It is possible that the high-mass FSs represent those that evolved to have a magnitude gap via mergers while the low-mass FSs are those that were formed with a magnitude gap. The systems that evolved to be FSs would contain FGs that have undergone numerous mergers and so would be expected to have large masses. They would also exist in rich systems and so the chances of them developing large magnitude gaps would be small. The systems that were formed as fossils, on the other hand, would contain FGs that had not undergone many (if any) mergers and so would be relatively less massive. Being poor systems, they are statistically more likely to have larger magnitude gaps.

The results from Figure 7 suggest that $z \sim 1$ BCGs cannot be the progenitors of low-redshift FSs without a major merger or an abnormal number of minor mergers at $z<1$. According to the fits in this figure, at $3 \mathrm{keV}$, an average FG has $M_{*}=8 \times 10^{11} M_{\odot}$ and an average BCG has $M_{*}=5 \times 10^{11} M_{\odot}$. The FG is therefore $60 \%$ more massive than the BCG. If a BCG was to undergo a single major merger to form an $\mathrm{FG}$, then this would require a merger ratio of less than 1:1.6 (i.e., a nearly equal-mass merger). However, a $z \sim 1$ galaxy undergoes a single major merger (merger ratios 1:1-1:4) in $\sim 8$ Gyr and a single minor merger (merger ratios 1:4-1:10) in $\sim 3$ Gyr (Lotz et al. 2011). The lookback time from $z=0.15$ (the mean redshift of the FG sample) to $z \sim 1$ is $\sim 6$ Gyr.

Essentially, the FG must be formed from the merger of two BCGs, a rare event at low redshift. In the SDSS, $90 \%$ of all major mergers occur between galaxies that are fainter than $L^{*}$ (Patton \& Atfield 2008). To have two BCGs merging requires the merger of two clusters - another rare event at low redshifts. $50 \%$ of massive halos at $z=0$ had their last major merger at $z \geqslant 1$ (Fakhouri et al. 2010). The above considerations suggest that FSs are not a phase of normal group/cluster evolution (von Benda-Beckmann et al. 2008).

Closely linked to the formation of the magnitude gap is the formation of the FG. Why are they among the most massive galaxies for a given system mass? Are FGs formed later than BCGs in non-FSs through gas-poor mergers (dry mergers; DíazGiménez et al. 2008)? Are they formed via gas-rich mergers (wet mergers; Khosroshahi et al. 2006b)? Or is it a combination of the two wet mergers occurring early with most of their mass being assembled later through dry mergers (Méndez-Abreu et al. 2012). The results of our stellar population analysis, which found no differences between FGs and BCGs, rule out late-time wet mergers. However, it is possible that the stellar population models we are using are unable to resolve such small differences.

The secret to determining the formation method of FGs and FSs may lie in the diffuse stellar component (DSC). The DSC is an important component of a cluster's overall luminosity and is composed of material that has been stripped from cluster galaxies during dynamical interactions (Feldmeier et al. 2002; Rudick et al. 2006, 2009). Anywhere from $10 \%$ to $40 \%$ of a system's total luminosity can be found in the DSC, with the quantity increasing with time due to mergers. It is likely that there is no universal DSC fraction, but that different groups/clusters will have different DSC levels, depending on their specific evolution and history (Murante et al. 2004; Rudick et al. 2006; Conroy et al. 2007). Also, differing dynamical interactions are found to create distinct structures within the DSC. For example, tidal streams are associated with fast, close encounters between a galaxy and the dominant galaxy. The DSC can provide a wealth of information on the dynamical history of both the dominant galaxy and the cluster itself (Rudick et al. 2011). 
The detection of structure in the DSC would indicate recent mergers and that the FG is still rapidly evolving at $z=0.2$ (e.g., Brough et al. 2011). The lack of structure (but the existence of DSC) would indicate that the FG has not evolved through major mergers since $z>1$ (e.g., Stott et al. 2008). On the other hand, a lack of DSC altogether would indicate that the FG formed its mass without major mergers (e.g., Mulchaey \& Zabludoff 1999).

\section{CONCLUSIONS}

Using X-ray data from the XCS combined with optical data from the SDSS, we have defined a sample of 17 FSs at $z \leqslant 0.25$, of which 14 have not been classified as such previously. This catalog represents not only an increase in the number of known FSs, but also an increase in the quality of the X-ray data used to study such systems. Using the data from XCS, we examine the X-ray scaling relations of FSs. For the FGs, we estimate stellar masses, ages, and metallicities using STARLIGHT and obtain star formation rates from MPA-JHU. Using these data we compare the stellar mass assembly and the stellar populations of FGs to two samples of BCGs, one optically selected and one X-ray selected. The main results from this paper are as follows.

1. FSs, i.e., systems with a large magnitude gap, have masses that range from those of galaxy groups to those of galaxy clusters.

2. At fixed halo mass, the stellar mass of the dominant galaxy in FSs is larger than those in non-FSs.

3. The fraction of light in the dominant galaxy, as well as the luminosity of the dominant galaxy, increases with magnitude gap for all galaxy groups and clusters.

A scenario whereby FSs form at high redshift and FGs grow to high masses through fast and efficient mergers could explain most of the results in this paper. A study of the intracluster light in both FSs and non-FSs could help to decide whether the above explanation is plausible or not.

The authors thank the anonymous referee who helped to improve the paper through a careful reading of the manuscript.

This work is based on observations obtained with $X M M$, an ESA science mission with instruments and contributions directly funded by the ESA Member States and NASA. It has made use of data or software provided by the US National Virtual Observatory, which is sponsored by the National Science Foundation; the NASA/IPAC Extragalactic Database (NED) which is operated by the Jet Propulsion Laboratory, California Institute of Technology, under contract with the National Aeronautics and Space Administration; and the SDSS. Funding for the SDSS and SDSS-II has been provided by the Alfred P. Sloan Foundation, the Participating Institutions, the National Science Foundation, the U.S. Department of Energy, the National Aeronautics and Space Administration, the Japanese Monbukagakusho, the Max Planck Society, and the Higher Education Funding Council for England. The SDSS Web site is http://www.sdss.org/. The SDSS is managed by the Astrophysical Research Consortium for the Participating Institutions. The Participating Institutions are the American Museum of Natural History, Astrophysical Institute Potsdam, University of Basel, University of Cambridge, Case Western Reserve University, University of Chicago, Drexel University, Fermilab, the Institute for Advanced Study, the Japan Participation Group, Johns Hopkins University, the Joint Institute for Nuclear Astrophysics, the Kavli Institute for Particle Astrophysics and Cosmology, the Korean Scientist Group, the Chi- nese Academy of Sciences (LAMOST), Los Alamos National Laboratory, the Max-Planck-Institute for Astronomy (MPIA), the Max-Planck-Institute for Astrophysics (MPA), New Mexico State University, Ohio State University, the University of Pittsburgh, the University of Portsmouth, Princeton University, the United States Naval Observatory, and the University of Washington.

Financial support for this project includes: The Science and Technology Facilities Council (STFC) through grants ST/F002858/1 and/or ST/I000976/1 (for E.L.-D., A.K.R., N.M., A.R.L., and M.S.), ST/H002391/1 and PP/E001149/1 (for C.A.C. and J.P.S.), and through a studentship (for N.M.), the University of KwaZulu-Natal and the Leverhulme Trust (for M.H.), FP7-PEOPLE-2007-4-3-IRG n 20218 (for B.H.), Fundação para a Ciência e a Tecnologia through the project PTDC/CTE-AST/64711/2006 (for P.T.P.V.), the Swedish Research Council (V.R.) through the Oskar Klein Centre for Cosmoparticle Physics (for M.S.), the U.S. Department of Energy, National Nuclear Security Administration by the University of California, Lawrence Livermore National Laboratory under contract No. W-7405-Eng-48 (for S.A.S.), and the National Science Foundation Cyber-Enabled Discovery and Innovation (CDI) through grant 0941742 (J.W.R.).

\section{APPENDIX A}

\section{THE ROBUSTNESS OF THE FOSSIL SYSTEM DEFINITION}

The most commonly used definition of an FS was first set out in Jones et al. (2003, the exact definition is given in the introduction). For a system to be classified as an FS in this study, it must have $L_{X \text {, bol }} \gtrsim 5 \times 10^{41} h_{70}^{-2} \mathrm{erg} \mathrm{s}^{-1}$ and a magnitude gap of 2.5 in the $r$ band between the brightest and the fourth brightest galaxies located within half the virial radius, which we denote as $\Delta m_{14}$. As mentioned in the introduction, one of the main reasons why there are so few confirmed FSs in the literature is the lack of high quality X-ray data. Low S/N $\mathrm{X}$-ray data make it extremely difficult to not only detect extended sources, but to also estimate their luminosity. It is for this reason that many samples are defined as "optical fossil" samples, i.e., samples that are only known to satisfy the optical criterion.

The source detection algorithm used in the XCS, the XCS Automated Pipeline Algorithm (XAPA), has made this project possible. Many difficulties can arise when trying to detect and measure X-ray sources, which usually have low counts: a point-spread function and sensitivity that varies over the instrument's field-of-view; deblending of point and extended sources; and background determination to name but a few (LD11, M11). However, once in possession of accurate $L_{X} \mathrm{~S}$, determining whether or not a system satisfies the X-ray criterion is a straightforward matter.

Determining whether or not the optical criterion is satisfied, however, is a more complicated matter. To be able to determine the magnitude gap of a system requires an estimate of the virial radius and redshift information for each of the galaxies, which is required to determine whether a galaxy is a member of the system or not. Many FS studies start with the Jones et al. (2003) definition and then modify it in various ways depending on circumstances. For example, if no estimate of the virial radius is available, then a fixed aperture may be used.

Studies have been performed on the effect the adopted definition of the magnitude gap has on the properties of FS (e.g., Dariush et al. 2010), but to date no study has been done 


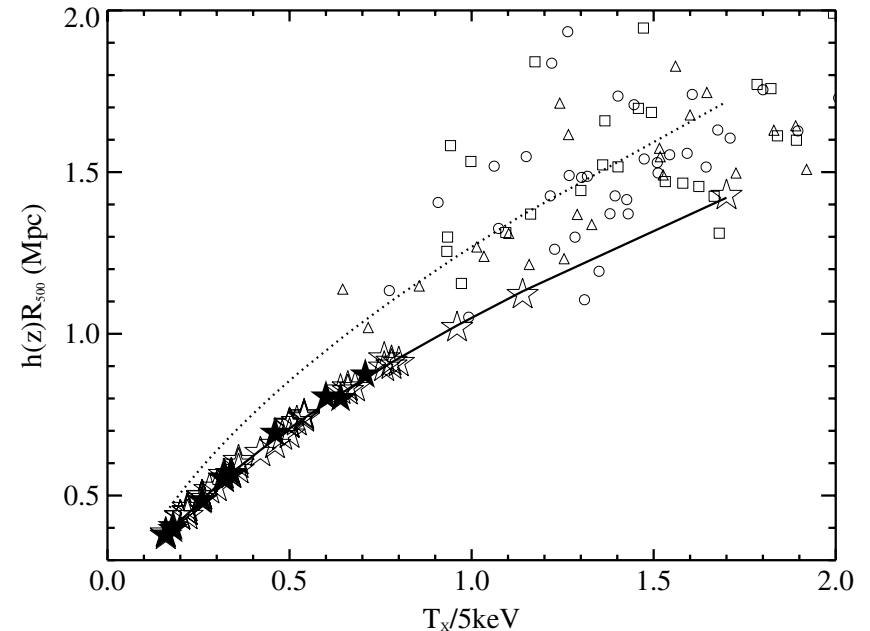

Figure 14. Comparison of the $R_{500}-T_{X}$ relation from Arnaud et al. (2005, solid line) to the relation determined from the $T_{X}$ and $R_{500}$ data from Mantz et al. (2010, dotted line). The stars are the data used in this paper (open for the XCS clusters and closed for the FS). Open circles, triangles, and squares are the BCS, REFLEX, MACS clusters respectively, where we show the core excised temperatures and radii from Mantz et al. (2010).

on the robustness of the FS definition. In this appendix, we test the robustness of our FS classifications by varying some of the parameters used during the search for FSs, e.g., the size of the redshift cuts. We also investigate how the results of this study change when we (1) use different radii to define the magnitude gap of our systems and (2) use the Jones et al. (2003) magnitude gap instead of the Dariush et al. (2010)

In this study, we relied on the redshift data provided by the SDSS to determine membership. Specifically, any galaxy with a spectroscopic redshift less than $\Delta c z=2000 \mathrm{~km} \mathrm{~s}^{-1}$ or a photometric redshift less than $\Delta z_{\text {phot }}=0.1$ away from the potential FG was considered a system member. Given that the average photometric redshift error for our sample was 0.04, this cut is quite generous and errs on the side of caution. The magnitude gap was then calculated based on the galaxies that populated the red sequence of the systems' CMD, within $\pm 0.2 \mathrm{mag}$ in color of the potential FG.

To test the robustness of our classifications, we varied some of these values to see how that would affect the classifications. We varied the spectroscopic-redshift cut in steps of $250 \mathrm{~km} \mathrm{~s}^{-1}$ up to $2000 \mathrm{~km} \mathrm{~s}^{-1}$ and the search radius in steps of $0.25 R_{200}$ from $0.25 R_{200}$ to $R_{200}$. We also considered two values for the color cut, 0.15 and $0.2 \mathrm{mag}$, and the photometric redshift cut, $\Delta z=0.1$ and 0.2 .

We find that the FS definition that we use here is robust to both the spectroscopic-redshift and photometric-redshift cuts, and the color-cut. The only quantity that it depends on is the size of the search radius. In all cases, reducing the search radius from $R_{200}$ to $0.25 R_{200}$ increases the magnitude gap, in some cases doubling it. Our sample is inherently robust to variations in the radius, since for many (more than half) of our systems we could have increased the search radius well beyond $0.5 R_{200}$ and the system would have still been classified as an FS. We also examined the effects on our measured magnitude gaps (and therefore FS classification) of the errors on $R_{200}$. Systems whose gaps change when varying $R_{200}$ within the errors are noted in the individual notes. These changes in $R_{200}$ would not reduce our measured $L_{X}$ s below that required to define an FS.

As mentioned in Section 2.2, our $R_{200} \mathrm{~s}$ are estimated from the $T_{X^{-}}-R_{200}$ relation found in Arnaud et al. (2005), which, although not as good as actually measuring $R_{200}$, is perfectly reasonable. There are, however, other methods of estimating $R_{200}$ when a direct measurement is not possible. Here, we investigate the effects that the choice of $R_{200}$ has on our results by repeating our analysis using four different estimates of $R_{200}$ : a fixed $0.35 \mathrm{Mpc}$ aperture, a fixed $0.5 \mathrm{Mpc}$ aperture, an $R_{200}$ estimated from the $N_{200}-R_{200}$ relation of Johnston et al. (2007), and an $R_{200}$ estimated from the $T_{X}-R_{200}$ derived from the data in Mantz et al. (2010). In Figure 14, we compare the $R_{500}-T_{X}$ relation from Arnaud et al. (2005, solid line) to the relation determined from the $T_{X}$ and $R_{500}$ data from Mantz et al. (2010, dotted line). The stars are the data used in this paper (open for the XCS clusters and closed for the FS). Open circles, triangles, and squares are the BCS, REFLEX, and MACS clusters, respectively, where we show the core excised temperatures and radii from Mantz et al. (2010). The fit to the Mantz et al. (2010) data produces a slightly larger $R_{500}$ than the Arnaud et al. (2005) fit ( $\sim 20 \%$ at $T_{X}=1.0 \mathrm{keV}$ ). We obtain the Mantz et al. (2010) $R_{200}$ estimates by scaling the $R_{200}$ estimates from Arnaud et al. (2005) by the ratio of the Mantz et al. (2010) and Arnaud et al. (2005) $R_{500}$ estimates. We find that this small shift in the $R_{200}-T_{X}$ scaling relations only weakly affects our sample definition and induces only a small amount of scatter into relevant figures. Similar effects are found when using the fixed $0.35 \mathrm{Mpc}$ apertures.

The other two estimators, the fixed $0.5 \mathrm{Mpc}$ aperture and the $N_{200}$ estimate, produce larger effects. Compared to our sample of $17 \mathrm{FSs}$, the former finds only 13 and the latter finds 21 , i.e., the number of FSs found at these extremes is $\pm 25 \%$ of that found in this study. The reason for the decrease in the case of the fixed $0.5 \mathrm{Mpc}$ aperture is due to the fact that almost half of our sample have $0.5 R_{200}$ less than $0.5 \mathrm{Mpc}$. The search radius is therefore larger than necessary and includes more galaxies, which results in a smaller magnitude gap. The reason for the increase in the case of the $R_{200}$ estimates from the $N_{200}-R_{200}$ relation is most likely due to the fact that true FSs, if they are the end-products of galaxy merging in groups/clusters, should have an $R_{200}$ that is too large for their richness compared to that of normal groups/clusters. Therefore, if such a relation based on normal groups/clusters is used, then the $R_{200}$ estimates (and the search radii) will be too small, resulting in larger magnitude gaps. Such a shift in sample size will have a significant impact on the results of an FS number density study.

These two estimators also introduce scatter into many of the tight relations that FSs were found to follow. This can be seen by comparing the left panel of Figure 15 to Figure 6 and the right panel to Figure 11. Figure 15 shows the optical luminosity of the galaxy system within $0.5 R_{200}$ as a function of $T_{X}$ (left panel) and the fraction of that light contained within the dominant galaxy as a function of $T_{X}$ (right). Note that using $N_{200}$ to estimate $R_{200}$, as we do here, changes the radius within which we calculate $L_{\text {tot }}$ (and therefore changes $L_{\mathrm{gal}} / L_{\text {tot }}$ ), but should not affect $T_{X}$ because at these radii the $T_{X}$ profile should be flat. In the case of the left plot, the scatter in this relation (for both FSs and XCS clusters) increases from 0.2 dex to 0.25 dex when using $N_{200}$ to estimate $R_{200}$. Note that by using $N_{200}$, we are also entirely removing any induced correlation between $L_{\text {tot }}$ and $T_{X}$ in Figure 6 , since the radius we used to calculate $L_{\text {tot }}$ is independent of $T_{X}$ (see Section 5). So the small increase in scatter in Figure 15 compared to Figure 6 could be from removing this correlation. In the right plot, apart from the increased scatter, we also see a boosting of the fraction at high $T_{X}$ because these systems are richer, and therefore a small decrease in radius can reduce the number of galaxies used to calculate the 

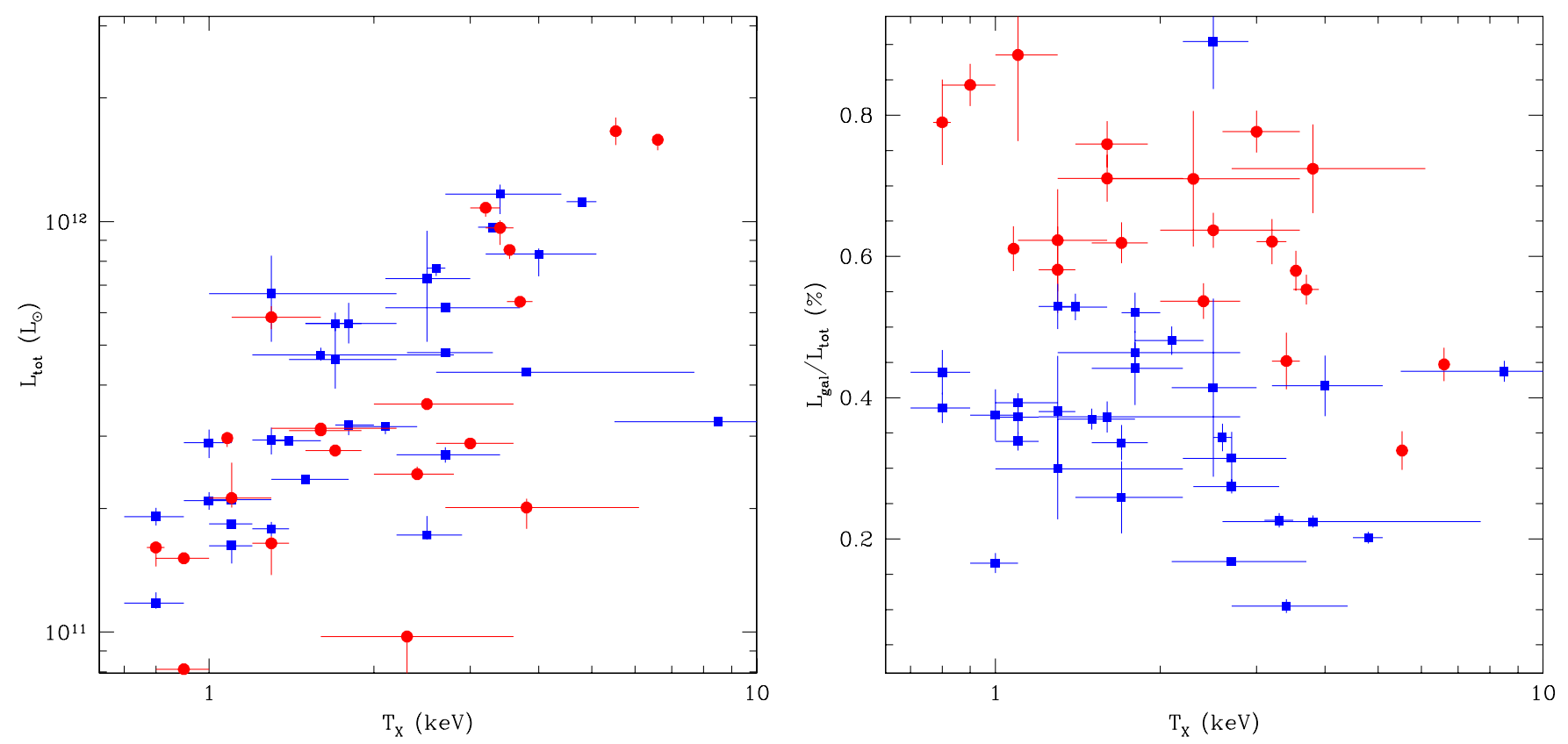

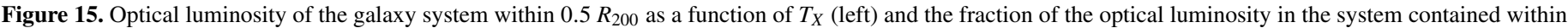

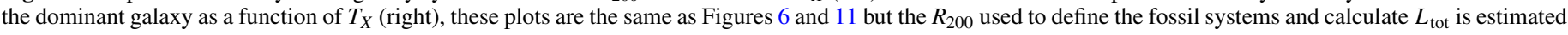
from the $N_{200}-R_{200}$ relation of Johnston et al. (2007) and they exhibit larger scatter.

(A color version of this figure is available in the online journal.)

magnitude gap more than for a low- $T_{X}$ system. This results in a smaller $L_{\mathrm{tot}}$ and a correspondingly larger fraction of the system light being contained in these dominant galaxies.

If we use the Jones et al. (2003) magnitude gap rather than the Dariush et al. (2010) magnitude gap, then we find that less systems are classified as an FS ( $\sim 30 \%$ less $)$ and we lose some statistical power. There are no changes to the overall results of this study, but we find that the tight trends we find for FSs (such as in Figure 11) are now more sparsely populated as some of our FSs have been re-classified as non-FSs. Interestingly, in the study of the magnitude gap made by Dariush et al. (2010), it was found that the Jones et al. (2003) magnitude gap was better at finding high-mass halos. We expected to lose mostly low-mass FSs when making this change but actually found that it was the intermediate mass FSs that fell out of the sample.

In summary, we find that our definition of an FS is robust to changes to the cuts used in redshift and color space but that it is sensitive to changes in $R_{200}$. We, therefore, tested various estimates of $R_{200}$ and found that the number of systems classified as an FS can vary by $\pm 25 \%$. All our results hold for the samples defined using various other estimates of $R_{200}$, but there is increased scatter in the correlations. Our results are also insensitive to our choice of magnitude gap definition. It is, therefore, important to have accurate estimates of $R_{200}$ when defining FS samples, especially if estimating their number density.

\section{APPENDIX B}

\section{NOTES ON INDIVIDUAL SYSTEMS}

In this appendix, we provide notes on each of the $17 \mathrm{FSs}$, along with SDSS images with XCS contours overlaid and CMDs. In the CMDs, green triangles are galaxies that, based on their SDSS spectroscopic redshift, are considered system members (i.e., $\Delta c z \leqslant 2000 \mathrm{~km} \mathrm{~s}^{-1}$ ) while red triangles are those that are not. Black circles are galaxies that, based on their SDSS photometric redshift, are considered system members (i.e., $\Delta z_{\text {phot }} \leqslant 0.1$ ). The blue diamonds mark the galaxies used to calculate $\Delta m_{12}$ and $\Delta m_{14}$. The dotted lines are the color cuts employed when calculating the magnitude gaps. Where possible, velocity dispersions have been calculated using the bi-weight estimator of Beers et al. (1990) and for those systems for which it was not possible to estimate a velocity dispersion values were obtained from the literature (if available).

\section{B.1. XMMXCS J015315.0+010214.2}

This $z=0.0597$ system is located at 01:53:15.0 +01:02:14.2 (Figure 16). The CMD shows an obvious RS, which all spectroscopic members lie on, and the magnitude gap is 2.7 based on SDSS photometric data; i.e., not all of the four brightest galaxies have a spectroscopic redshift. However, the two galaxies that only have photometric redshifts both lie on the RS as well. The X-ray temperature of the system is $T_{X}=1.1 \mathrm{keV}$ and the $\mathrm{X}$-ray emission peak lies $\sim 8.6 \mathrm{kpc}$ from the FG. The system is located near the edge of the SDSS footprint, but at a distance of $\sim 1.4 R_{200}$ both $\Delta m_{14}$ and $L_{\text {tot }}$ should be unaffected. The X-ray source is extended with $R_{200}=0.66 \mathrm{Mpc}$ or $\sim 30 R_{90}\left(R_{90}\right.$ is the radius within which $90 \%$ of the galaxy's light is contained, in this case the FG). The system has a velocity dispersion of $266 \mathrm{~km} \mathrm{~s}^{-1}$ based on 18 galaxies.

\section{B.2. XMMXCS J030659.8+000824.9}

This $z=0.0751$ system is located at 03:06:59.8 +00:08:24.9 (Figure 17). The CMD shows an RS and both spectroscopic members lay on it. The magnitude gap is 2.5 based on SDSS photometric data, and the galaxy that was excluded has a spectroscopic redshift that is $\sim 38,000 \mathrm{~km} \mathrm{~s}^{-1}$ away from the FG. Of the four galaxies used to calculate $\Delta m_{14}$, the two that only have photometric redshifts both lie on the RS as well. The X-ray emission has $T_{X}=2.3 \mathrm{keV}$ and the peak lies $\sim 26.2 \mathrm{kpc}$ from the FG. The system is located near the edge of the SDSS footprint, but at a distance of $\sim 7.0 R_{200}$ both $\Delta m_{14}$ and $L_{\text {tot }}$ are 

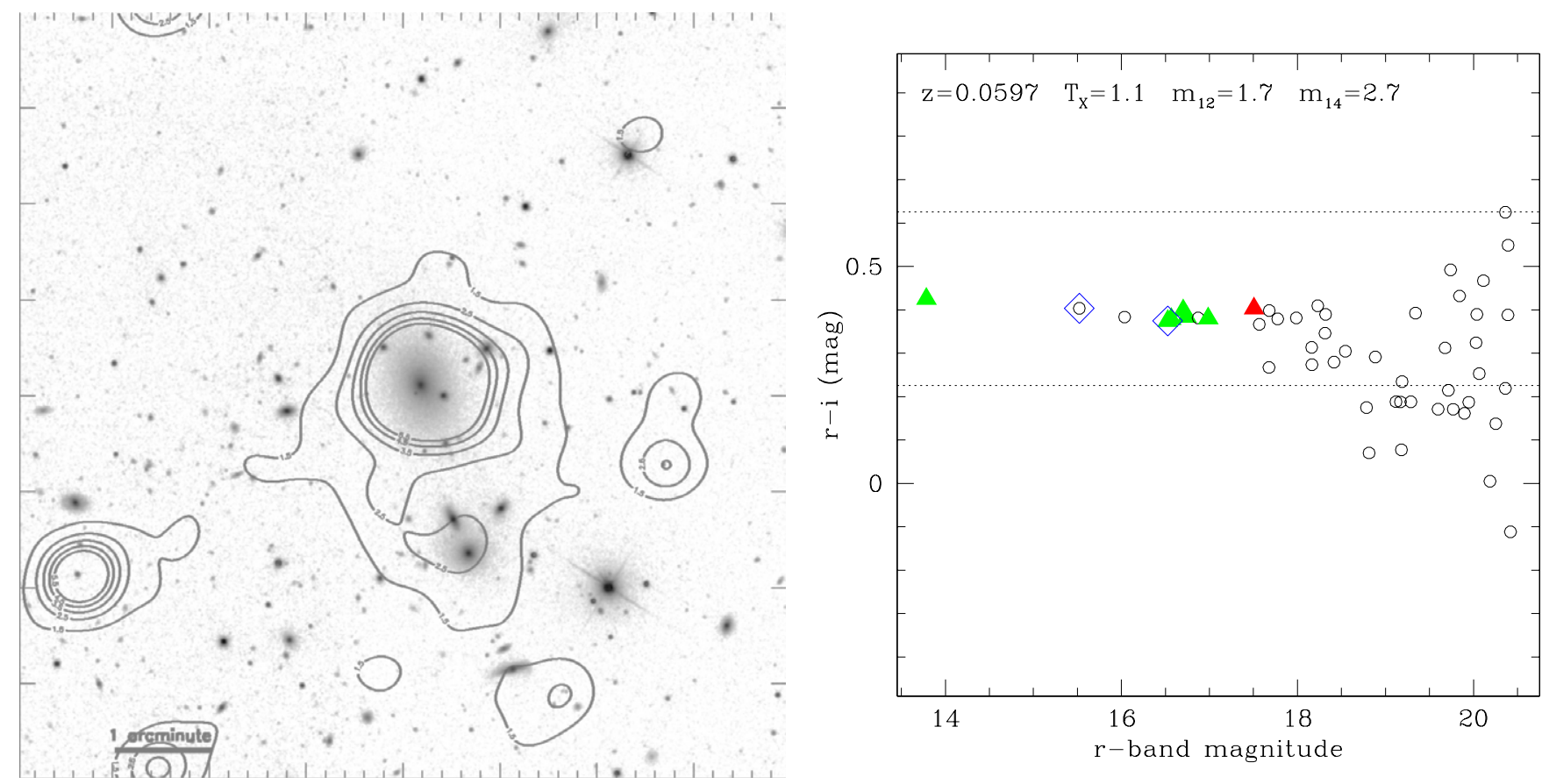

Figure 16. $z=0.0597$ system located at 01:53:15.0 +01:02:14.2.

(A color version of this figure is available in the online journal.)
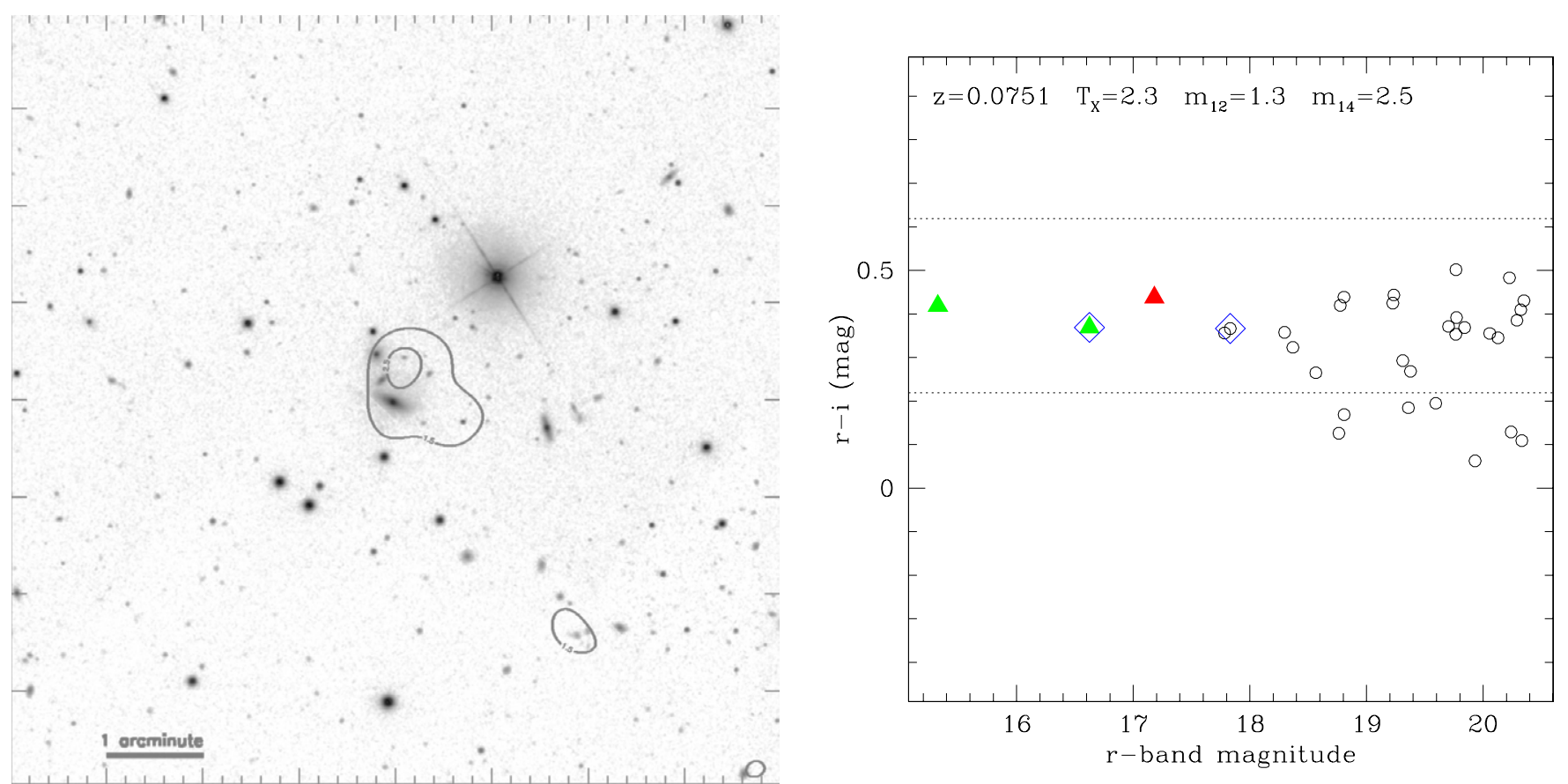

Figure 17. $z=0.0751$ system located at 03:06:59.8 +00:08:24.9.

(A color version of this figure is available in the online journal.)

unaffected. The X-ray source is extended, with $R_{200}=1.01 \mathrm{Mpc}$ or $\sim 90 R_{90}$ (the largest of any system). We note that this system is an outlier in many of the plots and, despite satisfying all the criteria necessary to be classified as an FS, we acknowledge that this classification is uncertain. The system has a velocity dispersion of $1082 \mathrm{~km} \mathrm{~s}^{-1}$ based on 13 galaxies.

\section{B.3. XMMXCS J073422.2+265143.9}

This $z=0.0796$ system is located at 07:34:22.2 +26:51:43.9 (Figure 18). The CMD shows an RS and all spectroscopic members lay on it. The magnitude gap is 3.0 based on SDSS spectroscopic data; i.e., all of the four brightest galaxies have a spectroscopic redshift. The X-ray emission has $T_{X}=1.1 \mathrm{keV}$ and the peak lies $\sim 1.5 \mathrm{kpc}$ from the FG. The system is located near the edge of the SDSS footprint, but at a distance of $\sim 10.0 R_{200}$ both $\Delta m_{14}$ and $L_{\text {tot }}$ are unaffected. The X-ray source is extended with $R_{200}=0.67 \mathrm{Mpc}$ or $\sim 30 R_{90}$. Reducing $R_{200}$ by its error would increase the magnitude gap by 0.5 . The system has a velocity dispersion of $411 \mathrm{~km} \mathrm{~s}^{-1}$ based on 18 galaxies. This system was classified as a fossil in Díaz-Giménez et al. (2008) and was an XMM target in a program to study FSs. 

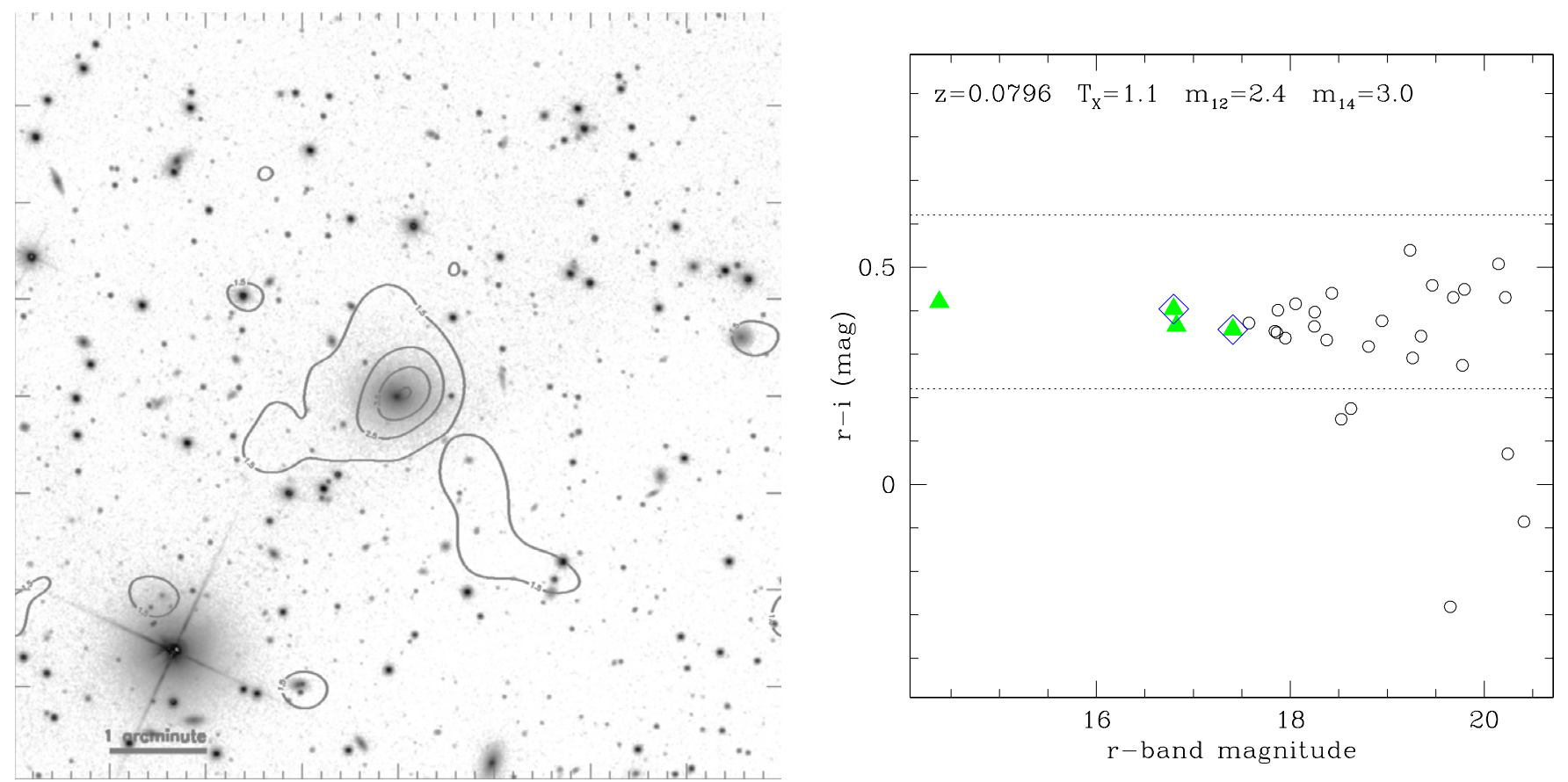

Figure 18. $z=0.0796$ system located at 07:34:22.2 +26:51:43.9.

(A color version of this figure is available in the online journal.)
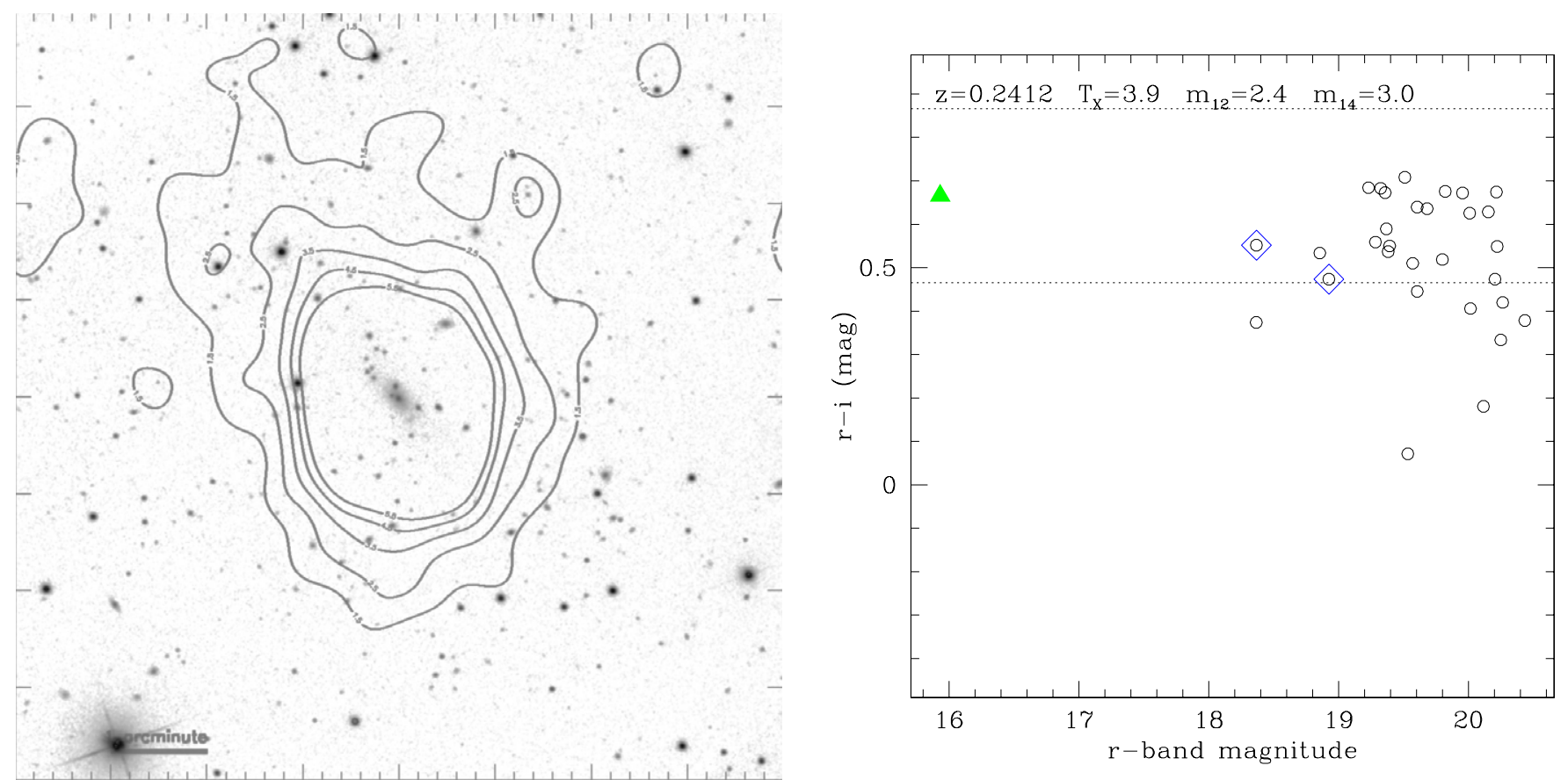

Figure 19. $z=0.2412$ system is located at 08:34:54.8 $+55: 34: 20.9$.

(A color version of this figure is available in the online journal.)

Díaz-Giménez et al. (2008) quote a virial radius of $1.6 \mathrm{Mpc}$ (twice as large as that found here) and a velocity dispersion of $551 \mathrm{~km} \mathrm{~s}^{-1}$.

\section{B.4. XMMXCS J083454.8+553420.9}

This $z=0.2412$ system is located at 08:34:54.8 +55:34:20.9 (Figure 19). There is only the slightest hint of an RS in the CMD. The magnitude gap is 3.0 based on SDSS photometric data. The X-ray emission has $T_{X}=3.9 \mathrm{keV}$ and the peak lies $\sim 3.3 \mathrm{kpc}$ from the FG. The X-ray source is extended, with $R_{200}=1.17 \mathrm{Mpc}$ or $\sim 30 R_{90}$.

\section{B.5. XMMXCS J092540.0+362711.1}

This $z=0.1121$ system is located at 09:25:40.0 +36:27:11.1 (Figure 20). The CMD shows an RS and all spectroscopic members lay on it. The magnitude gap is 2.8 based on SDSS 

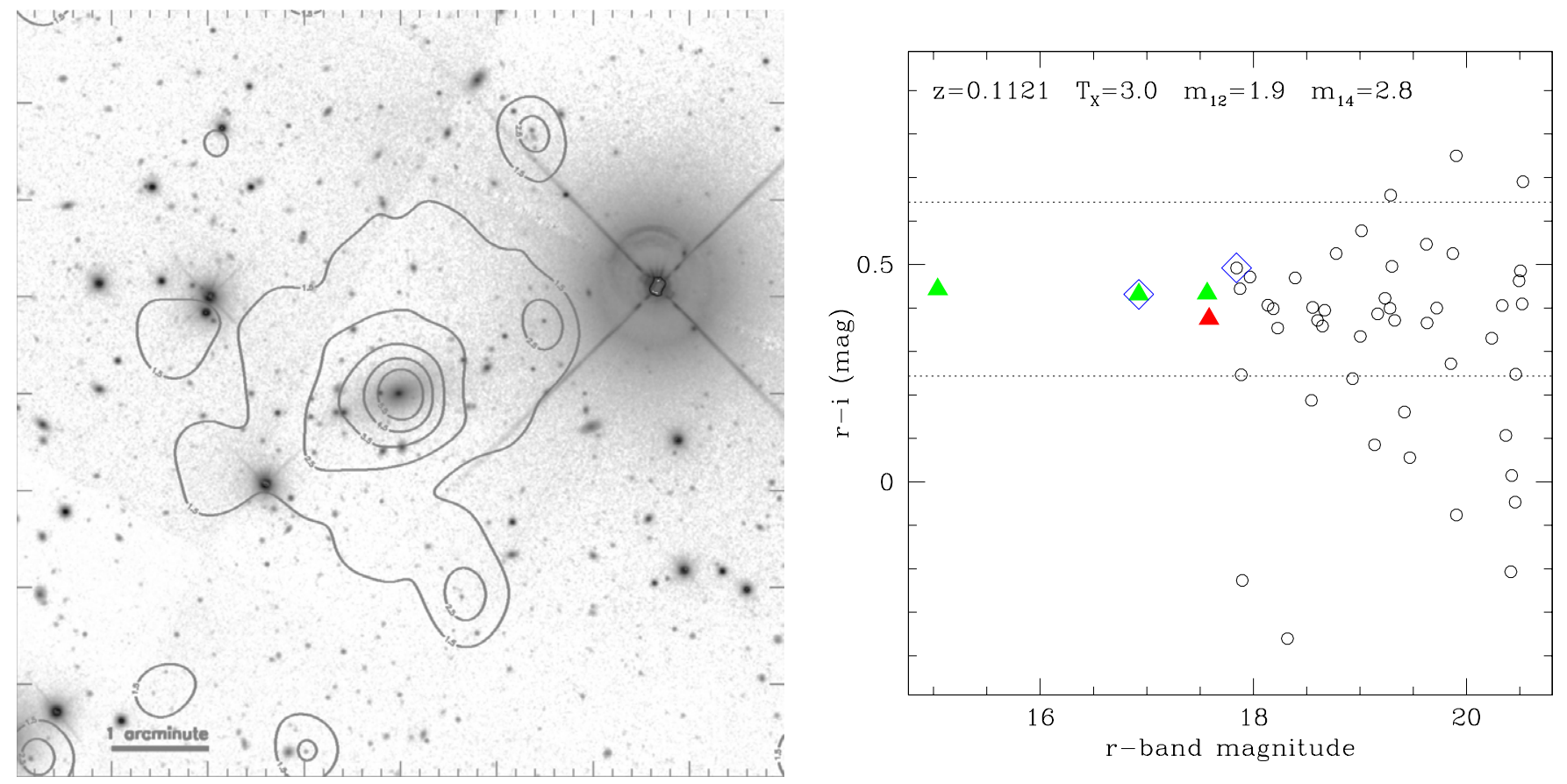

Figure 20. $z=0.1121$ system located at 09:25:40.0 +36:27:11.1

(A color version of this figure is available in the online journal.)
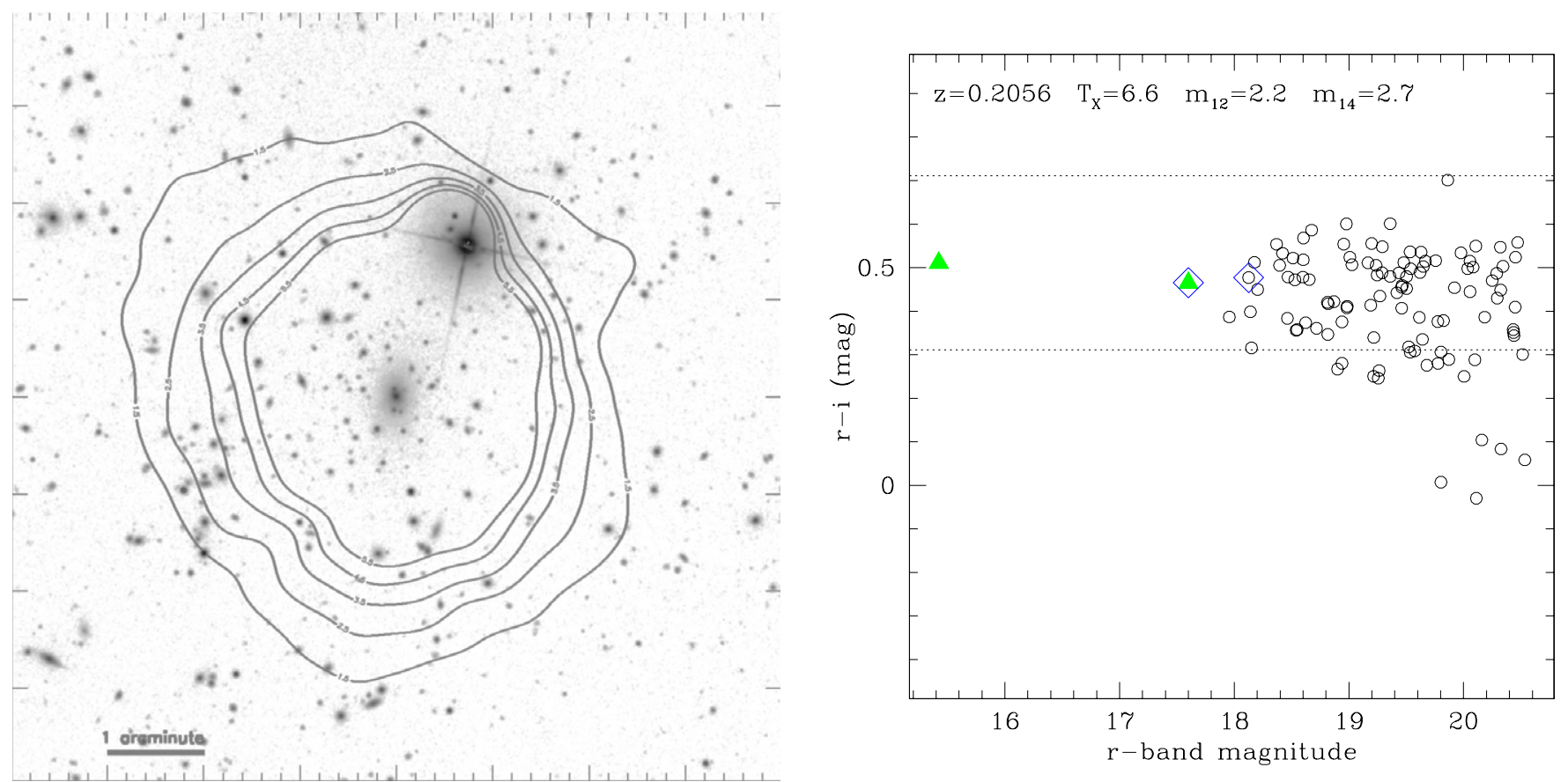

Figure 21. $z=0.2056$ system located at 10:17:03.6 +39:02:50.7

(A color version of this figure is available in the online journal.)

photometric data. The rejected galaxy lies $\sim 7800 \mathrm{~km} \mathrm{~s}^{-1}$ away from the FG. The X-ray emission has $T_{X}=3.0 \mathrm{keV}$ and the peak lies $\sim 26.1 \mathrm{kpc}$ from the FG. The $\mathrm{X}$-ray source is extended, with $R_{200}=1.14 \mathrm{Mpc}$ or $\sim 40 R_{90}$. Increasing $R_{200}$ by its error would decrease the magnitude gap by 0.3 but the system would still be classified as a fossil. We note that there is a bright galaxy just outside $0.5 R_{200}$ that would change $\Delta m_{14}$ if it were included. The system has a velocity dispersion of $435 \mathrm{~km} \mathrm{~s}^{-1}$ based on 22 galaxies.

\section{B.6. XMMXCS J101703.6+390250.7}

This $z=0.2056$ system is located at 10:17:03.6 +39:02:50.7 (Figure 21). The CMD shows the hint of an RS and both spectroscopic members lay on it. The magnitude gap is 2.7 based on SDSS photometric data. The X-ray emission has $T_{X}=6.6 \mathrm{keV}$ (the highest of any system) and the peak lies $\sim 4.6 \mathrm{kpc}$ from the FG. The X-ray source is extended with $R_{200}=1.63 \mathrm{Mpc}$ (the largest of any system) or $\sim 30 R_{90}$. We 

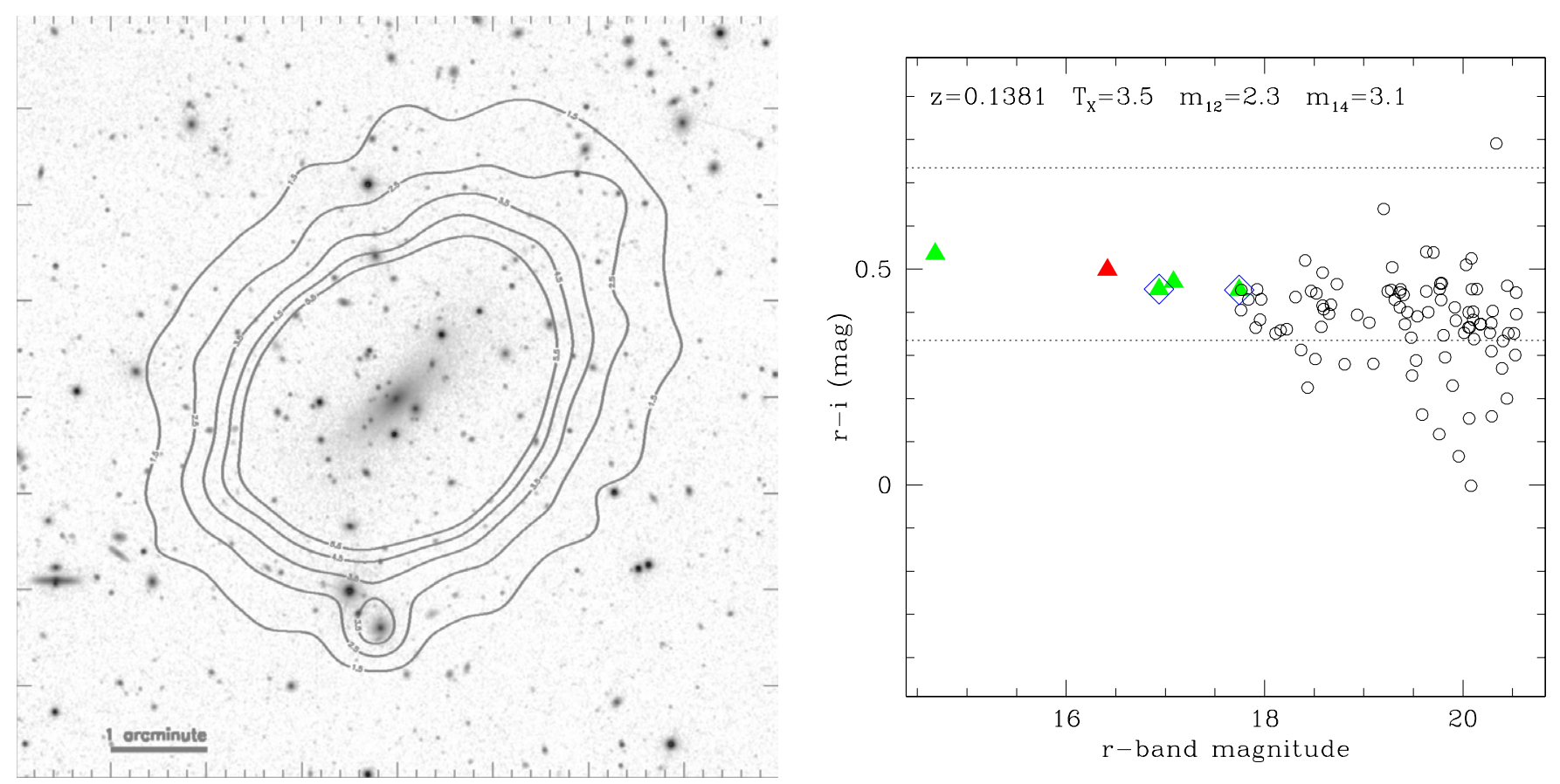

Figure 22. $z=0.1381$ system located at 01:40:44.4 +39:57:10.4

(A color version of this figure is available in the online journal.)

note that there is a bright galaxy just outside $0.5 R_{200}$ that would change $\Delta m_{14}$ if it were included. The system has a velocity dispersion of $1013 \mathrm{~km} \mathrm{~s}^{-1}$ based on 10 galaxies. This system is also known as A0963 and was an XMM target. From the literature, $L_{X}=6.1 \times 10^{44} \mathrm{erg} \mathrm{s}^{-1}$ (Soltan \& Henry 1983, half of that found here) and $\sigma=1350 \pm 200 \mathrm{~km} \mathrm{~s}^{-1}$ (Lavery \& Henry 1998). It is an X-ray lensing cluster that is unusually relaxed with $<5 \%$ substructure (Smith et al. 2005).

\section{B.7. XMMXCS J104044.4+395710.4}

This $z=0.1381$ system is located at 01:40:44.4+39:57:10.4 (Figure 22). The CMD shows an obvious RS, which all spectroscopic members lie on, and the magnitude gap is 3.1 based on SDSS spectroscopic data. The X-ray temperature of the system is $T_{X}=3.5 \mathrm{keV}$ and the $\mathrm{X}$-ray emission peak lies $\sim 3.2 \mathrm{kpc}$ from the FG. The X-ray source is extended, with $R_{200}=1.22 \mathrm{Mpc}$ or $\sim 25 R_{90}$. The system has a velocity dispersion of $1248 \mathrm{~km} \mathrm{~s}^{-1}$ based on 18 galaxies. This system is also known as A1068 and was an $X M M$ target. It is also a cooling-flow cluster with $L_{X}=5 \times 10^{44} \mathrm{erg} \mathrm{s}^{-1}$ (Quillen et al. 2008).

\section{B.8. XMMXCS J123024.3+111127.8}

This $z=0.1169$ system is located at 12:30:24.3+11:11:27.8 (Figure 23). The few galaxies that are in the vicinity of the FG all have redshifts (one spectroscopic and two photometric) similar to that of the FG. The CMD shows the hint of an RS, which both spectroscopic members lie on, and the magnitude gap is 3.5 (the largest of any system) based on SDSS photometric data. The X-ray temperature of the system is $T_{X}=0.8 \mathrm{keV}$ and the $\mathrm{X}$-ray emission peak lies $\sim 15.6 \mathrm{kpc}$ from the $\mathrm{FG}$. The $\mathrm{X}$-ray source is extended with $R_{200}=0.54 \mathrm{Mpc}$ or $\sim 20 R_{90}$, but is located close to the edge of an $X M M$ field. We note that there is a bright galaxy just outside $0.5 R_{200}$ that would change $\Delta m_{14}$ if it were included. No velocity dispersion could be measured for this system.

\section{B.9. XMMXCS J123338.5+374114.9}

This $z=0.1023$ system is located at 12:33:38.5 +37:41:14.9 (Figure 24). Again, there are very few galaxies in the vicinity of the FG, however, the CMD shows the hint of an RS. The only spectroscopic system member (the FG) lies on the RS and the magnitude gap is 3.2 based on SDSS photometric data. The rejected galaxy is $\sim 11,100 \mathrm{~km} \mathrm{~s}^{-1}$ away from the FG. The $\mathrm{X}$-ray temperature of the system is $T_{X}=0.9 \mathrm{keV}$ and the X-ray emission peak lies $\sim 21.6 \mathrm{kpc}$ from the FG. The X-ray source is extended, with $R_{200}=0.58 \mathrm{Mpc}$ or $\sim 30 R_{90}$. No velocity dispersion could be measured for this system.

\section{B.10. XMMXCS J124425.9+164758.0}

This $z=0.2346$ system is located at 12:44:25.9+16:47:58.0 (Figure 25). The FG in this system has a double core, therefore we cannot trust the sky-subtraction correction and so have not applied it. This means that the magnitude gap of 2.3 is a lower limit. If we applied the estimated correction, then the gap would be 3.3 and if we only applied the average correction from the other 16 FSs, then the gap would be 2.6, therefore we accept this as an FS. The X-ray temperature of the system is $T_{X}=1.3 \mathrm{keV}$ and the X-ray emission peak lies $\sim 25.8 \mathrm{kpc}$ from the FG. The $\mathrm{X}$-ray source is extended, with $R_{200}=0.63 \mathrm{Mpc}$ or $\sim 20 R_{90}$. No velocity dispersion could be measured for this system.

\section{B.11. XMMXCS J130749.6+292549.2}

This $z=0.2406$ system is located at 13:07:49.6+29:25:49.2 (Figure 26). There is the hint of an RS in the CMD, which the FG lies on, and the magnitude gap is 3.1 based on SDSS photometric data. There are no other spectroscopic objects in the vicinity of the FG, however, those objects that have photometric redshifts are at the same redshift. The X-ray temperature of the system is $T_{X}=3.2 \mathrm{keV}$ and the X-ray emission peak lies $\sim 18.9 \mathrm{kpc}$ from the FG. The X-ray source is extended, with $R_{200}=1.04 \mathrm{Mpc}$ or $\sim 25 R_{90}$. No velocity 

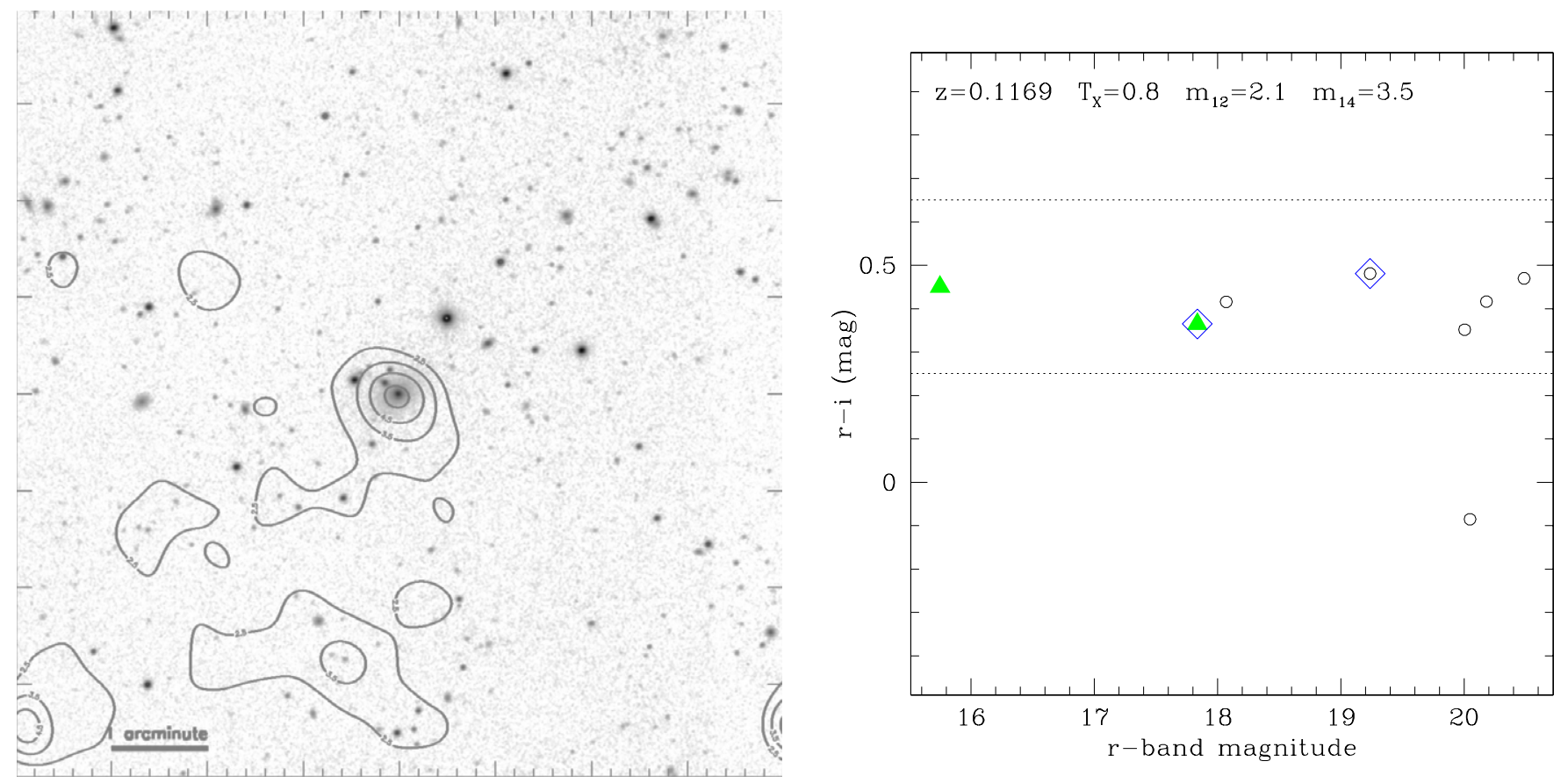

Figure 23. $z=0.1169$ system located at 12:30:24.3 +11:11:27.8.

(A color version of this figure is available in the online journal.)
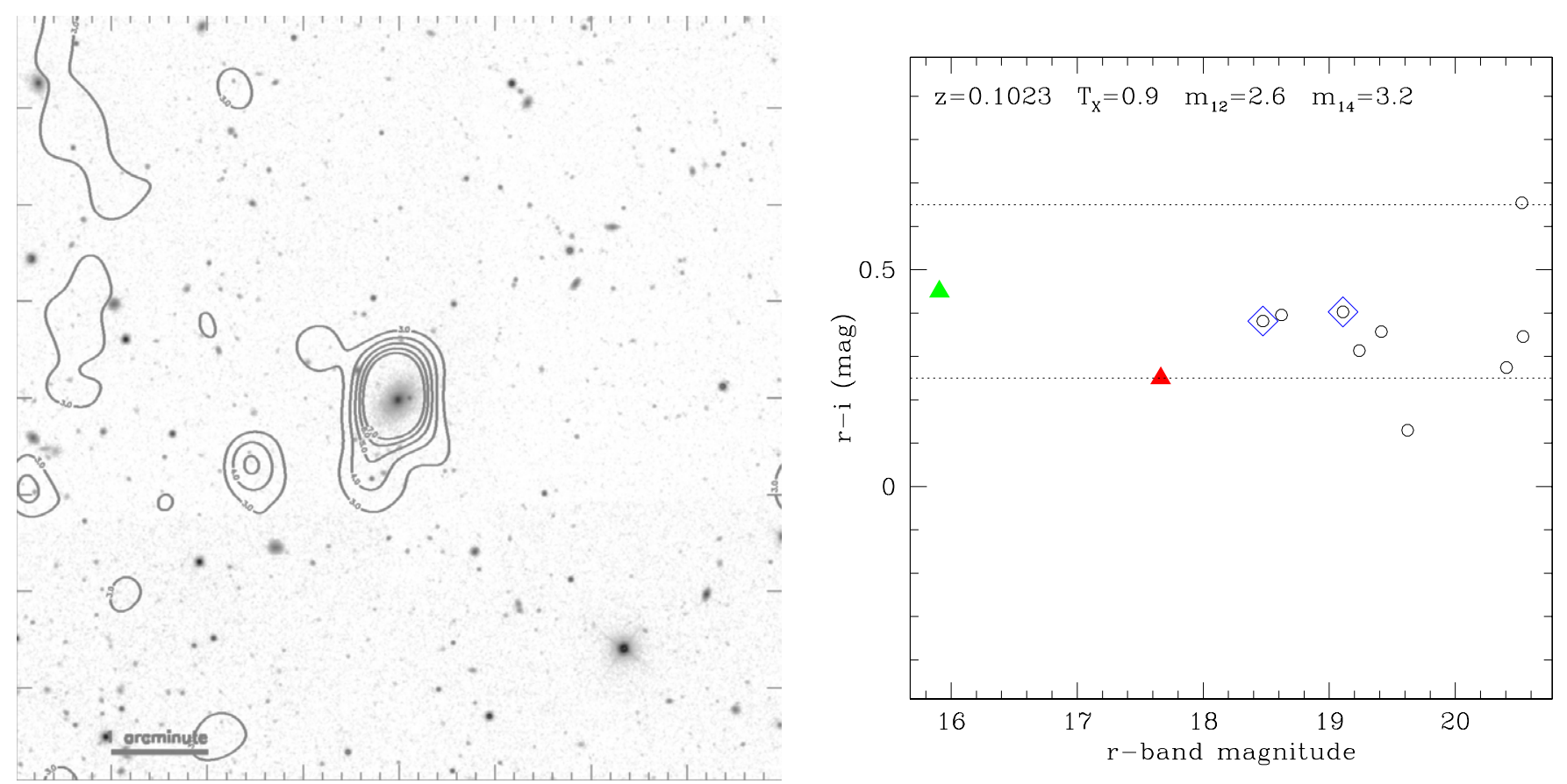

Figure 24. $z=0.1023$ system located at 12:33:38.5 +37:41:14.9.

(A color version of this figure is available in the online journal.)

dispersion could be measured for this system. This system is also known as $\mathrm{ZwCl} 1305.4+2941$. Gastaldello et al. (2008) find $T_{X}=3.17 \pm 0.19 \mathrm{KeV}$ (fully consistent with our measurement) and $L_{X, 500}=(1.25 \pm 0.16) \times 10^{44} h_{70}^{-2} \mathrm{erg} \mathrm{s}^{-1}$.

\section{B.12. XMMXCS J131145.1+220206.0}

This $z=0.1715$ system is located at 13:11:45.1 +22:02:06.0 (Figure 27). The CMD shows an obvious RS, which all spectroscopic members lie on, and the magnitude gap is 2.7 based on SDSS photometric data. The rejected galaxy is $\sim 50,000 \mathrm{~km} \mathrm{~s}^{-1}$ away from the FG. The X-ray temperature of the system is $T_{X}=3.4 \mathrm{keV}$ and the X-ray emission peak lies $\sim 95.3 \mathrm{kpc}$ from the FG. The X-ray source is extended, with $R_{200}=1.16 \mathrm{Mpc}$ or $\sim 25 R_{90}$. The system has a velocity dispersion of $362 \mathrm{~km} \mathrm{~s}^{-1}$ based on 10 galaxies. This system is also known as MaxBCG J197.94248+22.02702.

\section{B.13. XMMXCS J134825.6+580015.8}

This $z=0.1274$ system is located at 13:48:25.6 +58:00:15.8 (Figure 28). The CMD shows an obvious RS, which the only 

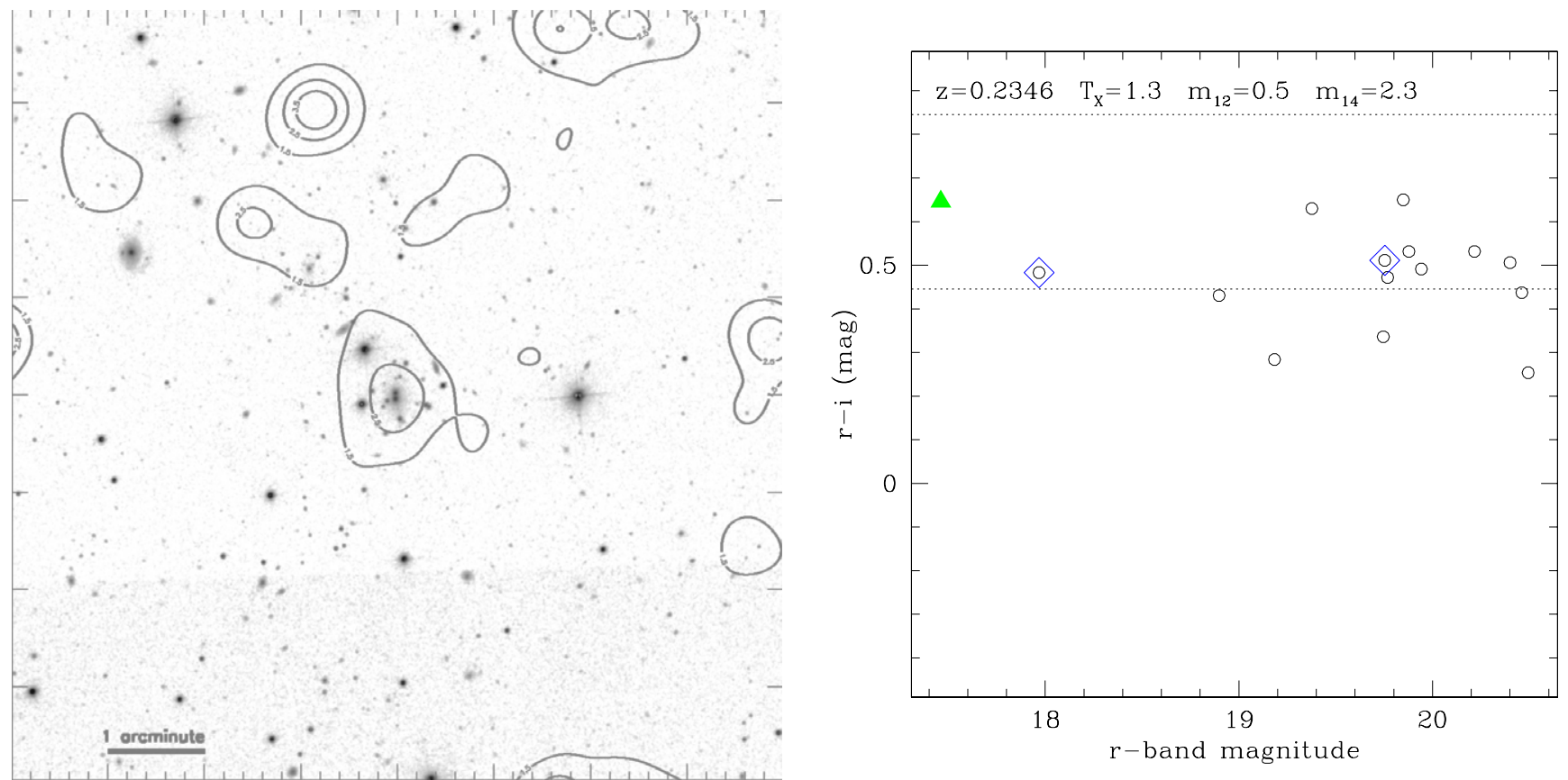

Figure 25. $z=0.2346$ system located at 12:44:25.9+16:47:58.0.

(A color version of this figure is available in the online journal.)
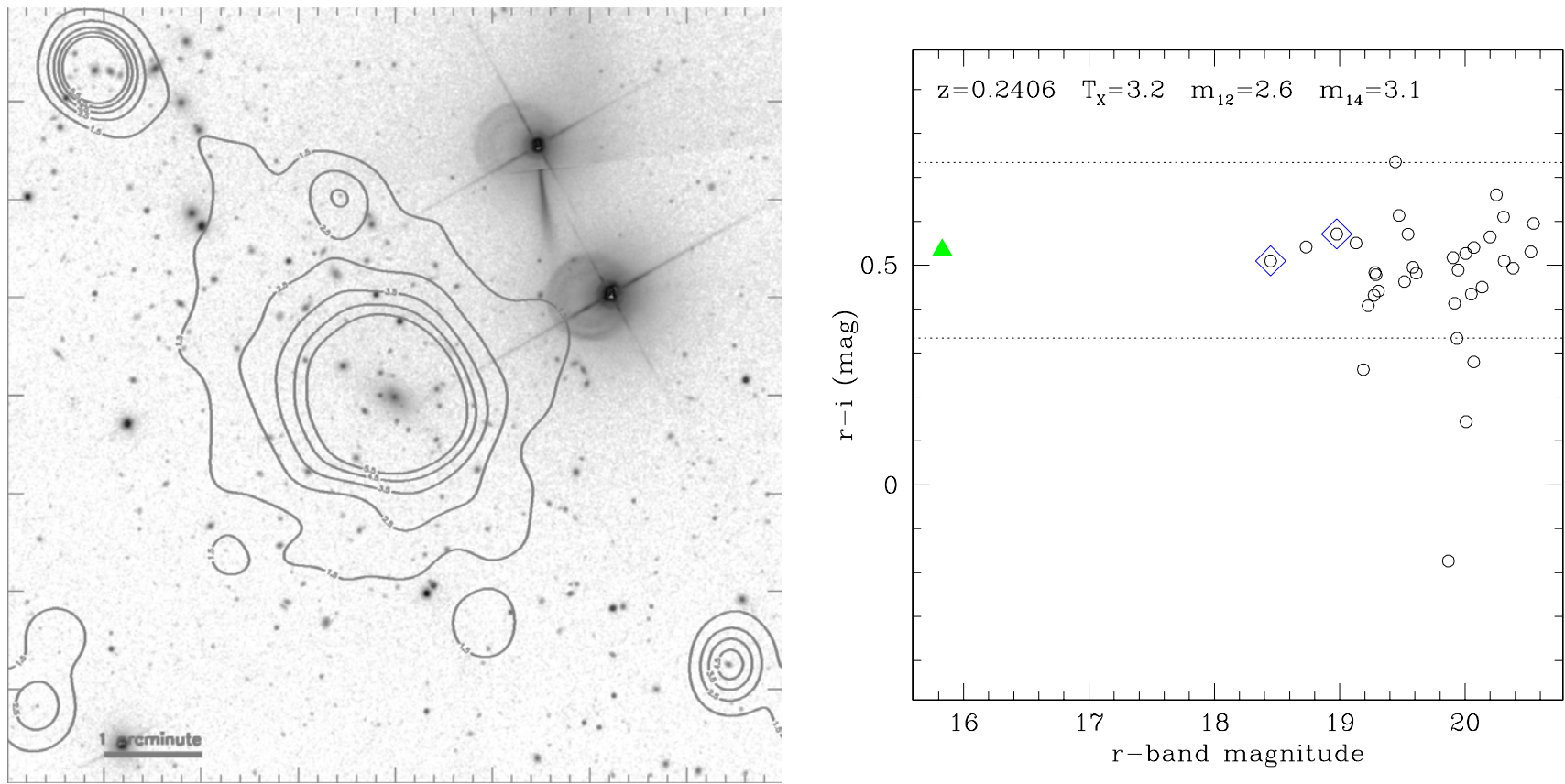

Figure 26. $z=0.2406$ system located at 13:07:49.6 +29:25:49.2.

(A color version of this figure is available in the online journal.)

spectroscopic member (the FG) lies on, and the magnitude gap is 2.6 based on SDSS photometric data. The rejected galaxy is $\sim 10,000 \mathrm{~km} \mathrm{~s}^{-1}$ away from the FG. The X-ray temperature of the system is $T_{X}=1.6 \mathrm{keV}$ and the $\mathrm{X}$-ray emission peak lies $\sim 7.4 \mathrm{kpc}$ from the FG. The X-ray source is extended, with $R_{200}=0.78 \mathrm{Mpc}$ or $\sim 20 R_{90}$, but it is located near the edge of the $X M M$ chip. The system has a velocity dispersion of $526 \mathrm{~km} \mathrm{~s}^{-1}$ based on five galaxies.

\section{B.14. XMMXCS J141627.7+231525.9}

This $z=0.1382$ system is located at 14:16:27.7 $+23: 15: 25.9$ (Figure 29). The CMD shows an obvious RS, which all spectro- scopic members lie on, and the magnitude gap is 2.9 based on SDSS photometric data. The rejected galaxy is $\sim 10,500 \mathrm{~km} \mathrm{~s}^{-1}$ away from the FG. The X-ray temperature of the system is $T_{X}=3.7 \mathrm{keV}$ and the X-ray emission peak lies $\sim 13.9 \mathrm{kpc}$ from the FG. The X-ray source is extended, with $R_{200}=1.25 \mathrm{Mpc}$ or $\sim 25 R_{90}$. The system has a velocity dispersion of $646 \mathrm{~km} \mathrm{~s}^{-1}$ based on 21 galaxies. This system was an XMM target and was classified as an FS in Jones et al. (2003) and subsequently studied in Voevodkin et al. (2010), Cypriano et al. (2006), and Khosroshahi et al. (2006a, 2006b). It is also known as $\mathrm{ZwCl} 1413.9+2330$. From the literature: $\Delta m_{12}=2.4$, $L_{X}=2.2 \times 10^{44} h_{50}^{-2} \mathrm{erg} \mathrm{s}^{-1}$, and $T_{X}=1.53 \pm 0.35 \mathrm{keV}$ (Jones 

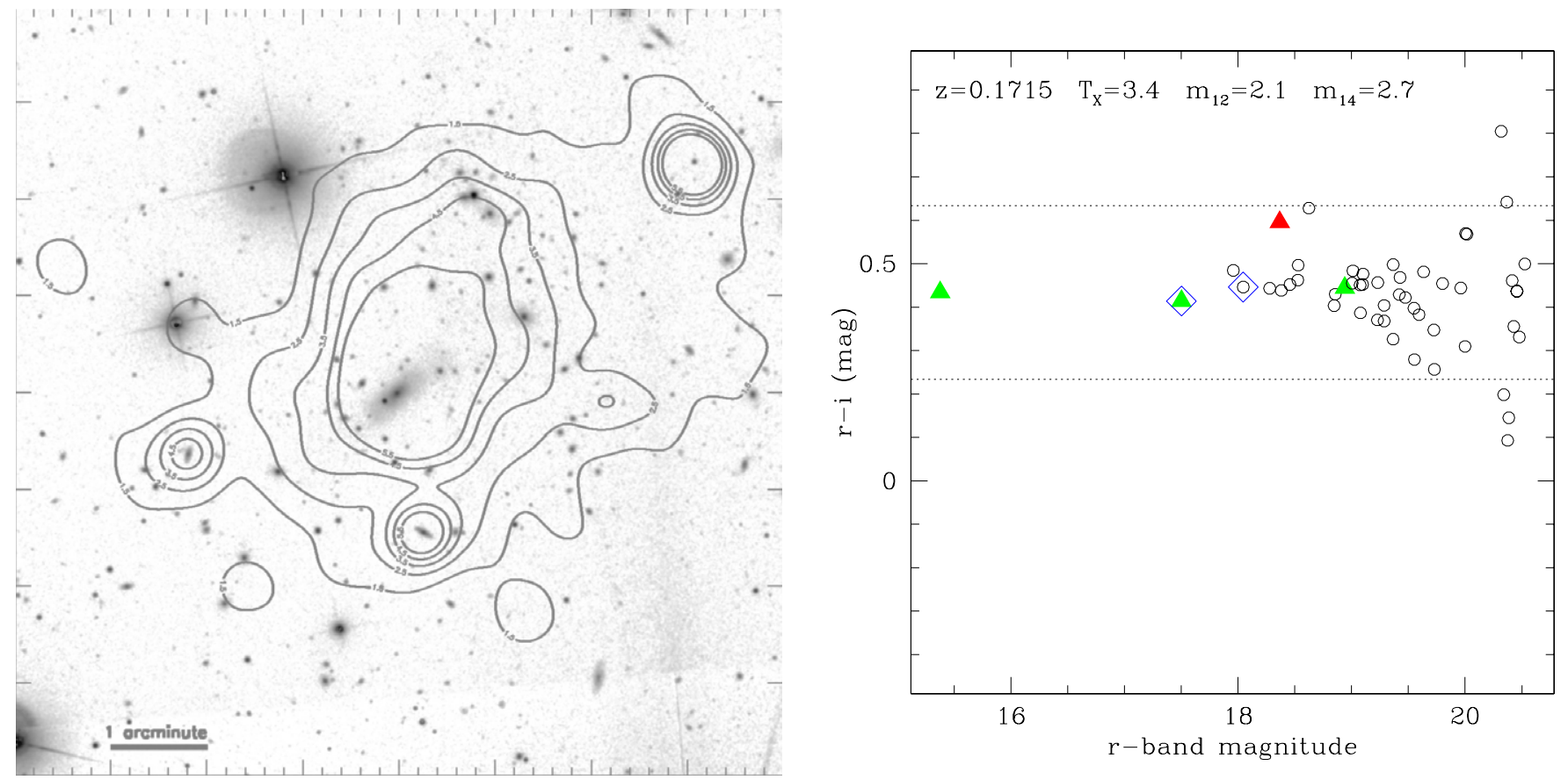

Figure 27. $z=0.1715$ system located at 13:11:45.1 +22:02:06.0.

(A color version of this figure is available in the online journal.)
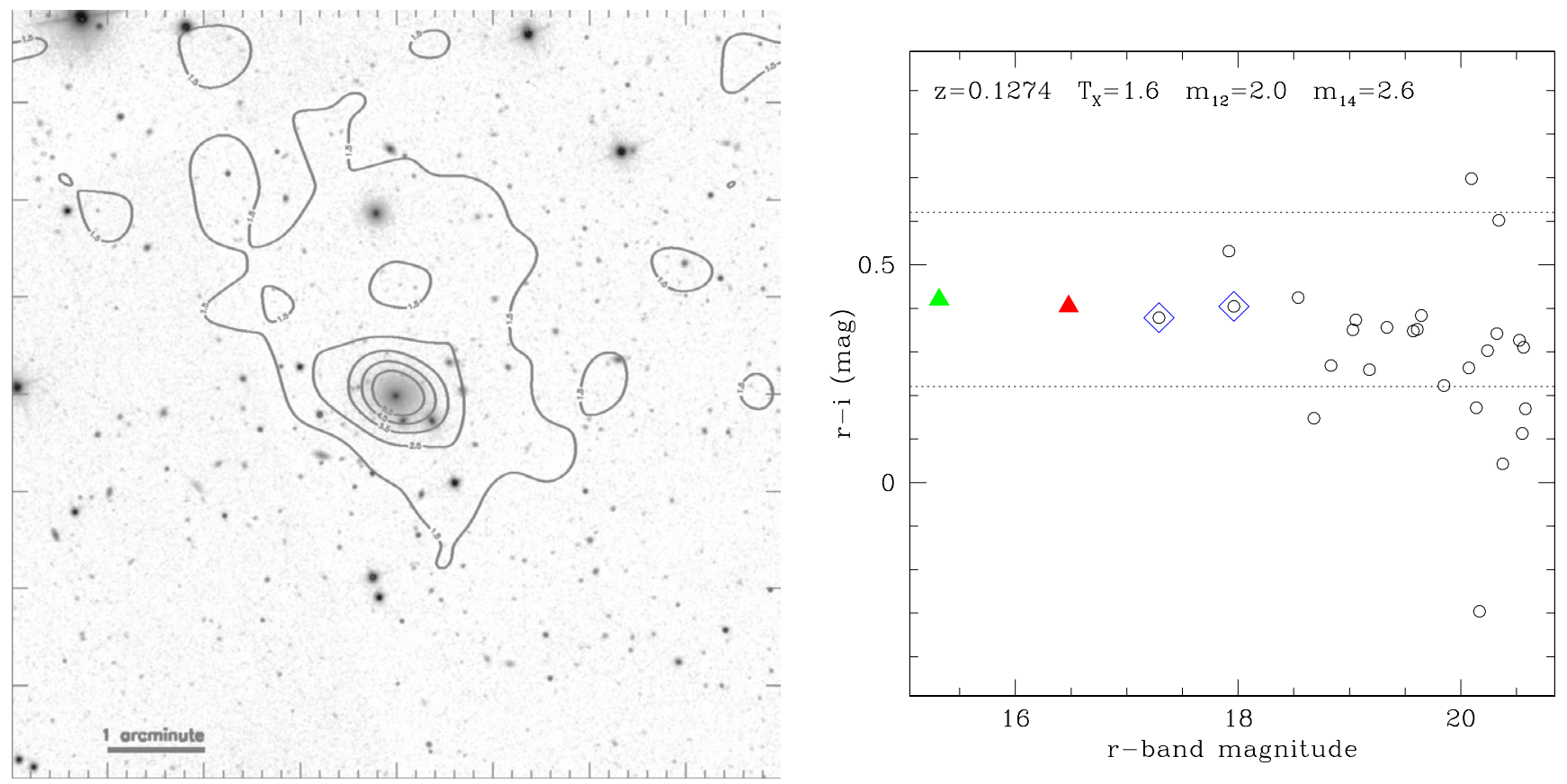

Figure 28. $z=0.1274$ system located at 13:48:25.6 +58:00:15.8.

(A color version of this figure is available in the online journal.)

et al. 2003); $L_{X}=1.11 \times 10^{44} h_{70}^{-2} \mathrm{erg} \mathrm{s}^{-1}$ (Cypriano et al. 2006); $T_{X} \sim 4 \mathrm{keV}, \sigma \sim 700 \mathrm{~km} \mathrm{~s}^{-1}$ (Khosroshahi et al. 2006a); $\Delta m_{12}=1.7 L_{X}=6.09 \times 10^{43} \mathrm{erg} \mathrm{s}^{-1} ; R_{500}=0.89 \mathrm{Mpc}$; and $\sigma=652 \mathrm{~km} \mathrm{~s}^{-1}$ (Voevodkin et al. 2010). This system appears to be associated with XMMXCS J141657.5+231239.2.

\section{B.15. XMMXCS J141657.5+231239.2}

This $z=0.1159$ system is located at $14: 16: 57.5+23: 12: 39.2$ (Figure 30). The CMD shows an RS and the magnitude gap is 3.1 based on SDSS photometric data. We note that there is a bright galaxy just outside $0.5 R_{200}$ that would change $\Delta m_{14}$ if it were included. The X-ray temperature of the system is $T_{X}=0.9 \mathrm{keV}$ and the X-ray emission peak lies $\sim 7.0 \mathrm{kpc}$ from the FG. The X-ray source is extended, with $R_{200}=0.56 \mathrm{Mpc}$ or $\sim 20 R_{90}$. No velocity dispersion could be measured for this system. This system appears to be associated with XMMXCS $\mathrm{J} 141627.7+231525.9$.

\section{B.16. ХMMXCS J160129.8+083856.3}

This $z=0.1875$ system is located at 16:01:29.8+08:38:56.3 (Figure 31 ). The CMD shows no sign of an RS and the magnitude 

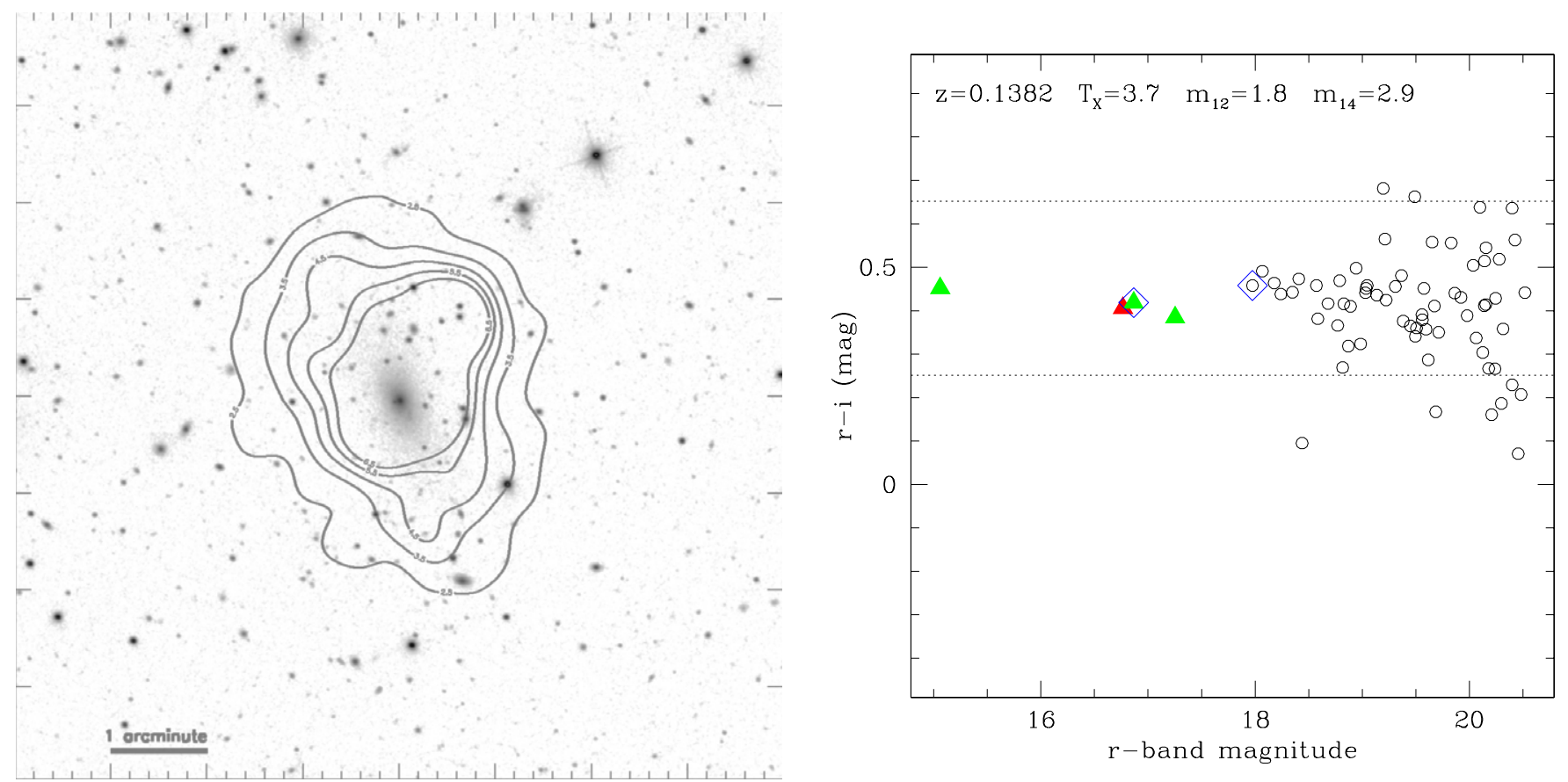

Figure 29. $z=0.1382$ system located at 14:16:27.7 +23:15:25.9.

(A color version of this figure is available in the online journal.)
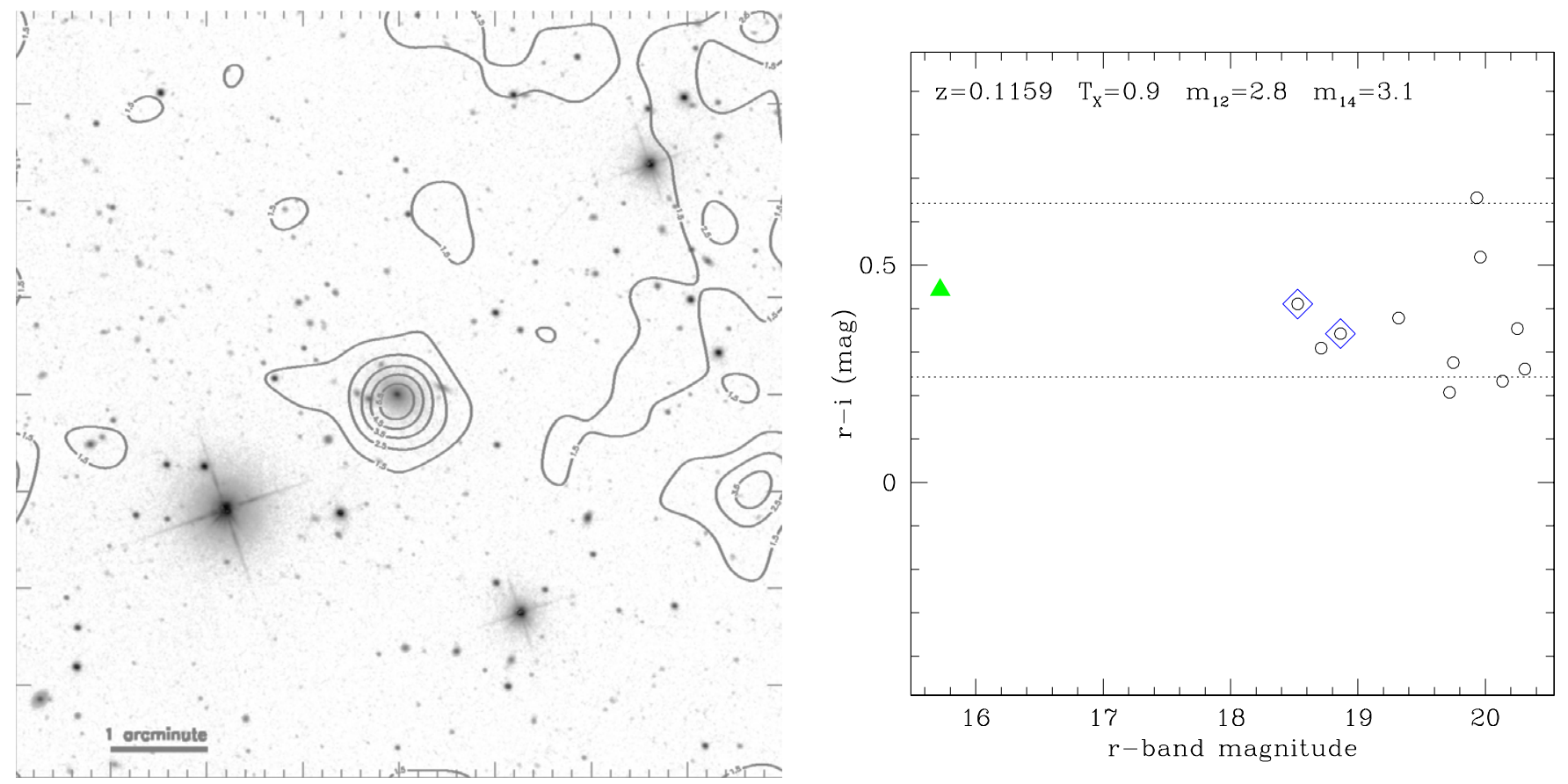

Figure 30. $z=0.1159$ system located at 14:16:57.5 +23:12:39.2.

(A color version of this figure is available in the online journal.)

gap is 3.1 based on SDSS photometric data. Increasing $R_{200}$ by its error would decrease the magnitude gap by 0.3 , but the system would still be classified as an FS. We note that there is a bright galaxy just outside $0.5 R_{200}$ that would change $\Delta m_{14}$ if it were included. The X-ray temperature of the system is $T_{X}=1.7 \mathrm{keV}$ and the X-ray emission peak lies $\sim 18.1 \mathrm{kpc}$ from the FG. The $\mathrm{X}$-ray source is extended, with $R_{200}=0.77 \mathrm{Mpc}$ or $\sim 35 R_{90}$. No velocity dispersion could be measured for this system.

\section{B.17. XMMXCS J172010.0+263724.7}

This $z=0.1596$ system is located at 17:20:10.0 +26:37:24.7 (Figure 32). The CMD shows an obvious RS, which all spectroscopic members lie on, and the magnitude gap is 2.45 based on SDSS spectroscopic data. This is close enough to 2.5 that we accept it as an FS. The two rejected galaxies are $2400 \mathrm{~km} \mathrm{~s}^{-1}$ and $20,000 \mathrm{~km} \mathrm{~s}^{-1}$ away from the FG. Reducing the spectroscopic 

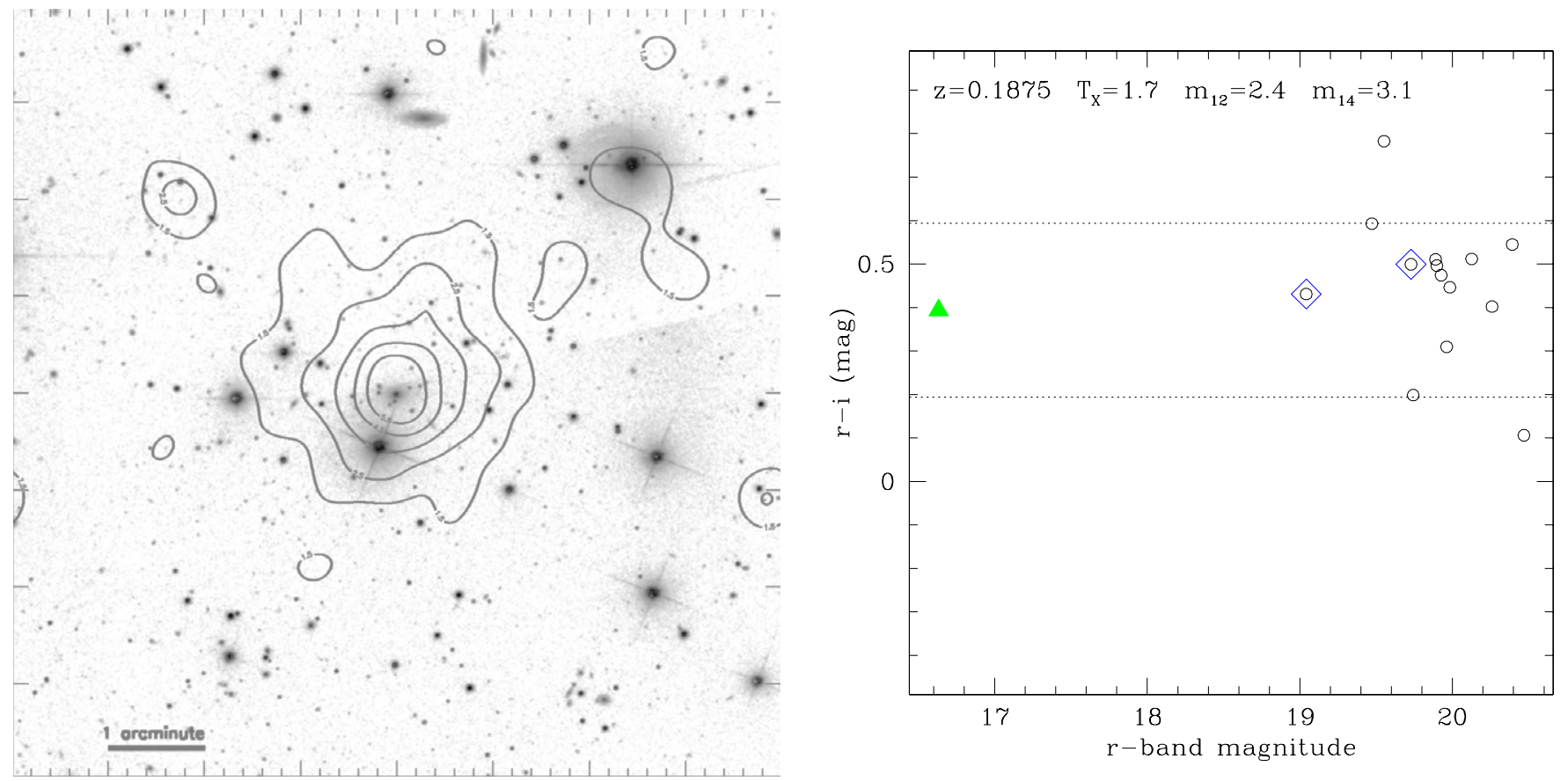

Figure 31. $z=0.1875$ system located at 16:01:29.8 +08:38:56.3.

(A color version of this figure is available in the online journal.)
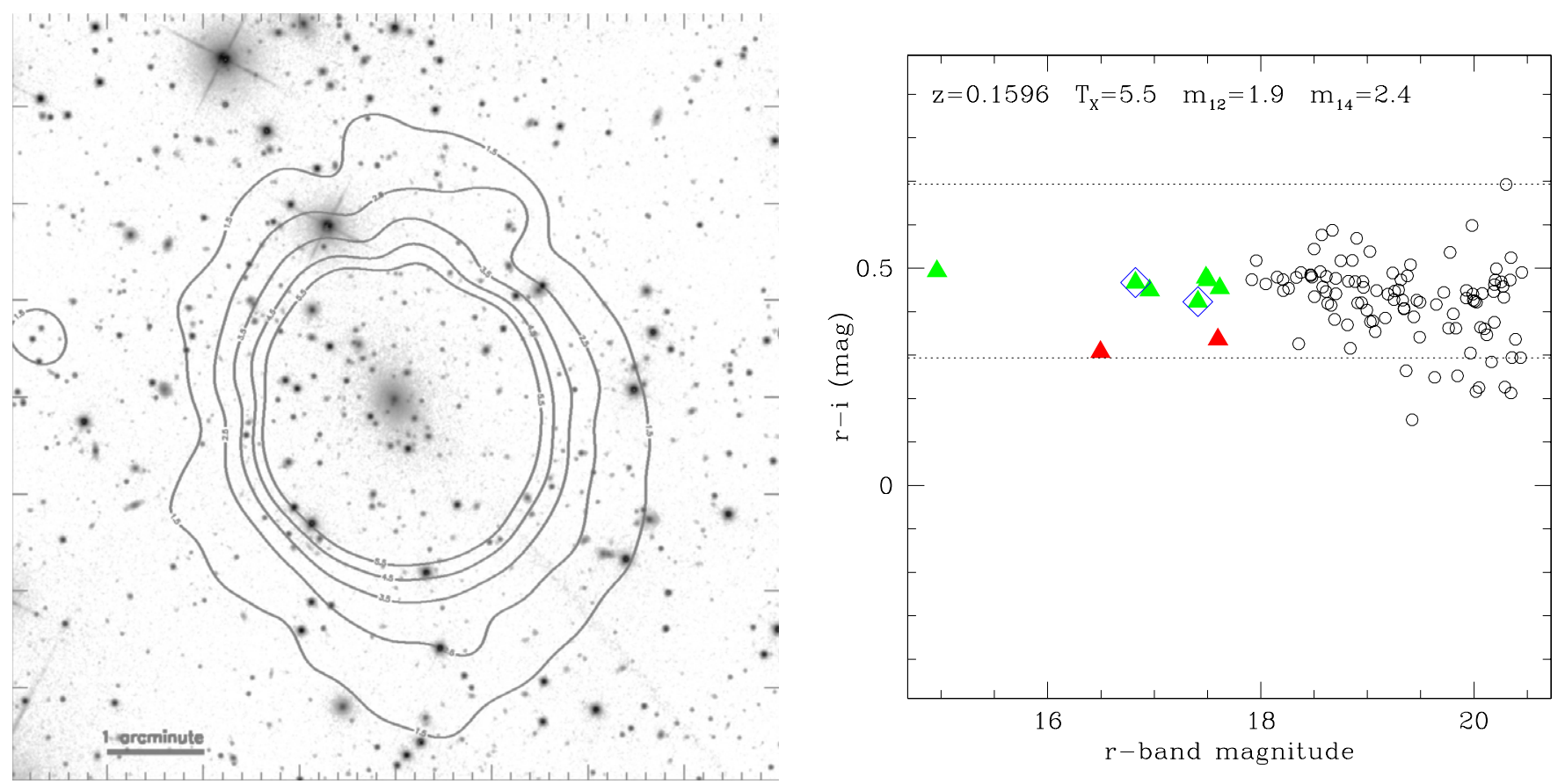

Figure 32. $z=0.1596$ system located at 17:20:10.0 +26:37:24.7

(A color version of this figure is available in the online journal.)

redshift cut to $1000 \mathrm{~km} \mathrm{~s}^{-1}$ would increase the magnitude gap by 0.4 . The X-ray temperature of the system is $T_{X}=5.5 \mathrm{keV}$ and the X-ray emission peak lies $\sim 20.2 \mathrm{kpc}$ from the FG. The system is located near the edge of the SDSS footprint, but at a distance of $\sim 17 R_{200}$ both $\Delta m_{14}$ and $L_{\text {tot }}$ are unaffected. The X-ray source is extended, with $R_{200}=1.54 \mathrm{Mpc}$ or $\sim 30 R_{90}$. This system was an $X M M$ target and was classified as an FS in Santos et al. (2007). It is also known as SDSS-C4 3072. The system has a velocity dispersion of $768 \mathrm{~km} \mathrm{~s}^{-1}$ based on 31 galaxies.

\section{REFERENCES}

Aguerri, J. A. L., Girardi, M., Boschin, W., et al. 2011, A\&A, 527, A143 Arnaud, M., Pointecouteau, E., \& Pratt, G. W. 2005, A\&A, 441, 893 Barnes, J. E. 1989, Nature, 338, 123

Beers, T. C., Flynn, K., \& Gebhardt, K. 1990, AJ, 100, 32

Bernardi, M., Hyde, J. B., Sheth, R. K., Miller, C. J., \& Nichol, R. C. 2007, AJ, 133, 1741

Bilir, S., Karaali, S., \& Tunçel, S. 2005, Astron. Nachr., 326, 321

Blanton, M. R., \& Roweis, S. 2007, AJ, 133, 734

Brinchmann, J., Charlot, S., White, S. D. M., et al. 2004, MNRAS, 351, 1151 
Brough, S., Collins, C. A., Burke, D. J., Lynam, P. D., \& Mann, R. G. 2005, MNRAS, 364, 1354

Brough, S., Collins, C. A., Burke, D. J., Mann, R. G., \& Lynam, P. D. 2002, MNRAS, 329, L53

Brough, S., Proctor, R., Forbes, D. A., et al. 2007, MNRAS, 378, 1507

Brough, S., Tran, K.-V., Sharp, R. G., von der Linden, A., \& Couch, W. J. 2011, MNRAS, 414, L80

Bruzual, A. G., \& Charlot, S. 1993, ApJ, 405, 538

Bruzual, G., \& Charlot, S. 2003, MNRAS, 344, 1000

Cash, W. 1979, ApJ, 228, 939

Cavaliere, A., \& Fusco-Femiano, R. 1976, A\&A, 49, 137

Charlot, S., \& Fall, S. M. 2000, ApJ, 539, 718

Charlot, S., Kauffmann, G., Longhetti, M., et al. 2002, MNRAS, 330, 876

Charlot, S., \& Longhetti, M. 2001, MNRAS, 323, 887

Cid Fernandes, R., Gu, Q., Melnick, J., et al. 2004, MNRAS, 355, 273

Cid Fernandes, R., Mateus, A., Sodré, L., Stasińska, G., \& Gomes, J. M. 2005, MNRAS, 358, 363

Collins, C. A., Stott, J. P., Hilton, M., et al. 2009, Nature, 458, 603

Conroy, C., Wechsler, R. H., \& Kravtsov, A. V. 2007, ApJ, 668, 826

Csabai, I., Dobos, L., Trencséni, M., et al. 2007, Astron. Nachr., 328, 852

Cui, W., Springel, V., Yang, X., De Lucia, G., \& Borgani, S. 2011, MNRAS, 416, 2997

Cypriano, E. S., Mendes de Oliveira, C. L., \& Sodré, L., Jr. 2006, AJ, 132, 514

Dariush, A., Khosroshahi, H. G., Ponman, T. J., et al. 2007, MNRAS, 382, 433

Dariush, A. A., Raychaudhury, S., Ponman, T. J., et al. 2010, MNRAS, 405, 1873

De Lucia, G., Springel, V., White, S. D. M., Croton, D., \& Kauffmann, G. 2006, MNRAS, 366, 499

De Lucia, G., \& Blaizot, J. 2007, MNRAS, 375, 2

Díaz-Giménez, E., Muriel, H., \& Mendes de Oliveira, C. 2008, A\&A, 490, 965

D’Onghia, E., Sommer-Larsen, J., Romeo, A. D., et al. 2005, ApJ, 630, L109

Dubinski, J. 1998, ApJ, 502, 141

Fakhouri, O., Ma, C.-P., \& Boylan-Kolchin, M. 2010, MNRAS, 406, 2267

Feldmeier, J. J., Mihos, J. C., Morrison, H. L., Rodney, S. A., \& Harding, P. 2002, ApJ, 575, 779

Ferland, G. J. 1996, University of Kentucky Internal Report

Gastaldello, F., Trevese, D., Vagnetti, F., \& Fusco-Femiano, R. 2008, ApJ, 673, 176

Herberich, E., Sikorski, J., \& Hothorn, T. 2010, A Robust Procedure for Comparing Multiple Means under Heteroscedasticity in Unbalanced Designs, ed. F. Rapallo (Public Library of Science) PLoS ONE 5(3): e9788, doi:10.1371/journal.pone.0009788

Johnston, D. E., Sheldon, E. S., Wechsler, R. H., et al. 2007, arXiv:0709.1159

Jones, L. R., Ponman, T. J., \& Forbes, D. A. 2000, MNRAS, 312, 139

Jones, L. R., Ponman, T. J., Horton, A., et al. 2003, MNRAS, 343, 627

Kauffmann, G., Heckman, T. M., White, S. D. M., et al. 2003, MNRAS, 341, 33

Khosroshahi, H. G., Jones, L. R., \& Ponman, T. J. 2004, MNRAS, 349, 1240

Khosroshahi, H. G., Maughan, B. J., Ponman, T. J., \& Jones, L. R. 2006a, MNRAS, 369, 1211

Khosroshahi, H. G., Ponman, T. J., \& Jones, L. R. 2006b, MNRAS, 372, L68

Khosroshahi, H. G., Ponman, T. J., \& Jones, L. R. 2007, MNRAS, 377, 595

Koester, B. P., McKay, T. A., Annis, J., et al. 2007, ApJ, 660, 221

La Barbera, F., de Carvalho, R. R., de la Rosa, I. G., et al. 2009, AJ, 137, 3942

Lauer, T. R., Gebhardt, K., Faber, S. M., et al. 2007, ApJ, 664, 226

Lavery, R. J., \& Henry, J. P. 1998, BAAS, 30, 864

Lin, Y.-T., \& Mohr, J. J. 2004, ApJ, 617, 879

Lloyd-Davies, E. J., Romer, A. K., Mehrtens, N., et al. 2011, MNRAS, 418, 14 (LD11)

Loh, Y.-S., \& Strauss, M. A. 2006, MNRAS, 366, 373
Lotz, J. M., Jonsson, P., Cox, T. J., et al. 2011, ApJ, 742, 103

Mantz, A., Allen, S. W., Ebeling, H., Rapetti, D., \& Drlica-Wagner, A. 2010, MNRAS, 406, 1773

Maughan, B. J. 2007, ApJ, 668, 772

Mehrtens, N., Romer, A. K., Lloyd-Davies, E. J., et al. 2011, arXiv:1106.3056 (M11)

Mendes de Oliveira, C. L., Cypriano, E. S., Dupke, R. A., \& Sodré, L. 2009, AJ, 138,502

Mendes de Oliveira, C. L., Cypriano, E. S., \& Sodré, L., Jr. 2006, AJ, 131, 158

Méndez-Abreu, J., Aguerri, J. A. L., Barrena, R., et al. 2012, A\&A, 537, A25

Miller, C. J., Nichol, Robert C., Reichart, D., et al. 2005, AJ, 130, 968

Miller, E. D., Rykoff, E., Dupke, R., et al. 2012, ApJ, 747, 94

Milosavljević, M., Miller, C. J., Furlanetto, S. R., \& Cooray, A. 2006, ApJ, 637, L9

Mulchaey, J. S., \& Zabludoff, A. I. 1999, ApJ, 514, 133

Murante, G., Arnaboldi, M., Gerhard, O., et al. 2004, ApJ, 607, L83

Nagamine, K., \& Loeb, A. 2003, New Astron., 8, 439

Osmond, J. P. F., \& Ponman, T. J. 2004, MNRAS, 350, 1511

Patton, D. R., \& Atfield, J. E. 2008, ApJ, 685, 235

Pipino, A., Szabo, T., Pierpaoli, E., MacKenzie, S. M., \& Dong, F. 2011, MNRAS, 417, 2817

Ponman, T. J., Allan, D. J., Jones, L. R., et al. 1994, Nature, 369, 462

Popesso, P., Böhringer, H., Romaniello, M., \& Voges, W. 2005, A\&A, 433, 415

Proctor, R. N., de Oliveira, C. M., Dupke, R., et al. 2011, MNRAS, 418, 2054

Quillen, A. C., Zufelt, N., Park, J., et al. 2008, ApJS, 176, 39

Richards, J. W., Freeman, P. E., Lee, A. B., \& Schafer, C. M. 2009, MNRAS, 399, 1044

Richardson, A. 2011, in International Statistical Review, Multiple Comparisons Using R, ed. F. Bretz, T. Hothorn, \& P. Westfall, 79, 297 (Wiley Online Library)

Romer, A. K., Viana, P. T. P., Liddle, A. R., \& Mann, R. G. 2001, ApJ, 547, 594

Rudick, C. S., Mihos, J. C., Frey, L. H., \& McBride, C. K. 2009, ApJ, 699, 1518

Rudick, C. S., Mihos, J. C., \& McBride, C. 2006, ApJ, 648, 936

Rudick, C. S., Mihos, J. C., \& McBride, C. K. 2011, ApJ, 732, 48

Ruszkowski, M., \& Springel, V. 2009, ApJ, 696, 1094

Salim, S., Rich, R. M., Charlot, S., et al. 2007, ApJS, 173, 267

Santos, W. A., Mendes de Oliveira, C., \& Sodré, L., Jr. 2007, AJ, 134, 1551

Schirmer, M., Suyu, S., Schrabback, T., et al. 2010, A\&A, 514, A60

Shaffer, J. P. 1995, Annu. Rev. Psychol., 46, 561

Smith, G. P., Kneib, J.-P., Smail, I., et al. 2005, MNRAS, 359, 417

Sommer-Larsen, J. 2006, MNRAS, 369, 958

Soltan, A., \& Henry, J. P. 1983, ApJ, 271, 442

Stott, J. P., Collins, C. A., Sahln, M., et al. 2010, ApJ, 718, 23

Stott, J. P., Edge, A. C., Smith, G. P., Swinbank, A. M., \& Ebeling, H. 2008, MNRAS, 384, 1502

Tavasoli, S., Khosroshahi, H. G., Koohpaee, A., Rahmani, H., \& Ghanbari, J. 2011, PASP, 123, 1

Tremonti, C. A., Heckman, T. M., Kauffmann, G., et al. 2004, ApJ, 613, 898

Voevodkin, A., Borozdin, K., Heitmann, K., et al. 2010, ApJ, 708, 1376

Voevodkin, A., Miller, C. J., Borozdin, K., et al. 2008, ApJ, 684, 204

von Benda-Beckmann, A. M., D’Onghia, E., Gottlöber, S., et al. 2008, MNRAS, 386,2345

von der Linden, A., Best, P. N., Kauffmann, G., \& White, S. D. M. 2007, MNRAS, 379, 867

Whiley, I. M., Aragón-Salamanca, A., De Lucia, G., et al. 2008, MNRAS, 387, 1253

White, S. D. M., \& Rees, M. J. 1978, MNRAS, 183, 341

Wu, X.-P., Xue, Y.-J., \& Fang, L.-Z. 1999, ApJ, 524, 22

York, D. G., Adelman, J., Anderson, J. E., Jr., et al. 2000, AJ, 120, 1579

Zibetti, S., Pierini, D., \& Pratt, G. W. 2009, MNRAS, 392, 525 